\title{
Universality of conductivity in interacting graphene
}

\author{
A. Giuliani \\ Università di Roma Tre, L.go S. L. Murialdo 1, 00146 Roma - Italy \\ V. Mastropietro \\ Università di Roma Tor Vergata, V.le della Ricerca Scientifica, 00133 Roma - Italy \\ M. Porta \\ ETH, Wolfgang Pauli Strasse 27, 8093 Zürich - Switzerland

\begin{abstract}
The Hubbard model on the honeycomb lattice describes charge carriers in graphene with short range interactions. While the interaction modifies several physical quantities, like the value of the Fermi velocity or the wave function renormalization, the a.c. conductivity has a universal value independent of the microscopic details of the model: there are no interaction corrections, provided that the interaction is weak enough and that the system is at half filling. We give a rigorous proof of this fact, based on exact Ward Identities and on constructive Renormalization Group methods.
\end{abstract}

\section{INTRODUCTION AND MAIN RESULTS}

The effects of interactions in quantum many body theory at low temperatures pose notoriously difficult problems; in certain cases the physical properties of the system are radically changed with respect to the non interacting situation, while in other cases, like in the socalled Fermi liquids, a simple modification or renormalization of the physical quantities is expected. There is, however, a very small group of phenomena which are universal; the physical quantities appear to be protected from any renormalization due to the interactions and their values do not dependent on the details of the model, but rather upon fundamental constants. A celebrated example is provided by the Quantum Hall Effect, in which the value of the plateaus only depend on the von Klitzing constant $h / e^{2}$ and not on the material parameters; universality appears to be related to topological invariance [1, 2] or to the presence

of Ward Identities [3]. Other examples of universal phenomena come from the physics of superconductivity, in which the magnetic quantum $h / e$ plays an important role. 
In recent times, evidence for universality has been observed in the conductivity of graphene, a one atom thick layer of graphite. Its electronic properties can be well described in terms of a tight-binding model of electrons hopping from one site to a neighboring one of a honeycomb lattice, but often this model is approximated by an effective one expressed in terms of massless Dirac fermions in the two-dimensional continuum [4]. Recent optical measurements [5] show that at half-filling and small temperatures, if the frequency is in a range well inside the temperature and the band-width, the a.c. conductivity is essentially constant and equal, up to a few percent, to $\sigma_{0}=\frac{e^{2}}{h} \frac{\pi}{2}$. Such value only depends on the von Klitzing constant and not on the material parameters, like the Fermi velocity; it is apparently universal, at least inside the experimental precision.

Is graphene a.c. conductivity truly universal? Theoretically, the computation of the conductivity in the absence of interactions gives exactly the value $\sigma_{0}=\frac{e^{2}}{h} \frac{\pi}{2}$, both in the idealized Dirac description [6] and even in the more realistic tight binding model [7]. However, since truly universal phenomena are quite rare in condensed matter, it is important to understand whether this apparently universal value is just an artifact of the idealized description in terms of non-interacting fermions or rather it is a robust property still valid in the presence of electron-electron interactions, which are certainly present and expected to play a role in real graphene. Such a question has been studied in the physical literature, but contradictory results have been found, see [8-10]. The reason for this is that the Dirac approximation, which works well for the free gas, is not very accurate in the presence of interactions: the corrections to the conductivity are expressed by logarithmically divergent integrals. One can argue that such divergence is spurious, just an artifact of the Dirac approximation, and that a regularization must be adopted to cure it (in the tight binding model, the lattice provides a natural cut-off); however, the results appear to be regularization-dependent and no unique predictions can be drawn.

In this paper we consider the Hubbard model on the honeycomb lattice, as a model of monolayer graphene with screened interactions, and we prove that the a.c. conductivity has the universal value $\sigma_{0}=\frac{e^{2}}{h} \frac{\pi}{2}$ even in presence of interactions: the interaction corrections to the conductivity are vanishing, provided that the interaction is weak enough and the system is in the half filled band case. Remarkably, the presence of the lattice and its symmetries is essential to get the result. The idea of the proof is based on the two main ingredients: (i) exact lattice Ward Identities (WI) relating the current-current, vertex and 
2-point functions; (ii) the fact that the interaction-dependent corrections to the Fourier transform of the current-current correlations are differentiable with continuous derivative (in contrast, the free part is continuous and not differentiable at zero frequency).

The paper is organized in the following way. In Section $1 \mathrm{~A}$ we describe the model, in Section 1B we derive the Ward Identities, in Section 1C (and Appendix A) we perform the computation of the conductivity in the non interacting case, and in Section 1D we present the proof of our main result, under some regularity assumptions on the currentcurrent correlations. The proof of these regularity properties, the full description of the expansion for the current-current correlations and vertex functions, as well as the proof of convergence of this expansion, is given in Section 2 (Appendix $\mathrm{B}$ and $\mathrm{C}$ collect the proof of some symmetry properties extensively used in Section 21).

\section{A. The model and the observables}

We consider electrons on a two-dimensional honeycomb lattice interacting via a local Hubbard interaction, as a model describing the charge carriers in graphene. Its ground state properties at half-filling, including the asymptotic behavior of the correlations at large distances, have already been analyzed in [11, 12]. In this subsection we recall the definition of the model and introduce some of the key observables (density and current), which will allow us to define the conductivity, i.e., the quantity of main interest in this paper.

The fermionic fields and the Hamiltonian. Let $\Lambda=\left\{n_{1} \vec{l}_{1}+n_{2} \vec{l}_{2}: n_{1}, n_{2}=0, \ldots, L-1\right\}$ be a periodic triangular lattice of period $L$, with basis vectors: $\vec{l}_{1}=\frac{1}{2}(3, \sqrt{3}), \vec{l}_{2}=\frac{1}{2}(3,-\sqrt{3})$. Let us denote by $\Lambda_{A}=\Lambda$ and $\Lambda_{B}=\Lambda+\vec{\delta}_{i}$ the $A$ - and $B$-sublattices of the honeycomb lattice, with $\vec{\delta}_{i}$ the n.n. vectors defined as:

$$
\vec{\delta}_{1}=(1,0), \quad \vec{\delta}_{2}=\frac{1}{2}(-1, \sqrt{3}), \quad \vec{\delta}_{3}=\frac{1}{2}(-1,-\sqrt{3}) .
$$

If $\vec{A}$ and $\vec{B}$ are two arbitrary constant vectors, we introduce creation and annihilation fermionic operators for electrons sitting at the sites of the $A$ - and $B$ - sublattices with spin 
index $\sigma=\uparrow \downarrow$ as

$$
\begin{array}{ll}
a_{\vec{x}, \sigma}^{ \pm}=L^{-2} \sum_{\vec{k} \in \mathcal{B}_{L}} e^{ \pm i \vec{k}(\vec{x}-\vec{A})} \hat{a}_{\vec{k}, \sigma}^{ \pm}, & \vec{x} \in \Lambda_{A}, \\
b_{\vec{x}, \sigma}^{ \pm}=L^{-2} \sum_{\vec{k} \in \mathcal{B}_{L}} e^{ \pm i \vec{k}(\vec{x}-\vec{B})} \hat{b}_{\vec{k}, \sigma}^{ \pm}, & \vec{x} \in \Lambda_{B},
\end{array}
$$

where $\mathcal{B}_{L}=\left\{\vec{k}=n_{1} \vec{G}_{1} / L+n_{2} \vec{G}_{2} / L: 0 \leq n_{i}<L\right\}$, with $\vec{G}_{1,2}=\frac{2 \pi}{3}(1, \pm \sqrt{3})$, is the first Brillouin zone; note that in the thermodynamic limit $L^{-2} \sum_{\vec{k} \in \mathcal{B}_{L}} \rightarrow|\mathcal{B}|^{-1} \int_{\mathcal{B}} d \vec{k}$, with $\mathcal{B}=\left\{\vec{k}=\xi_{1} \vec{G}_{1}+\xi_{2} \vec{G}_{2}: \xi_{i} \in[0,1)\right\}$ and $|\mathcal{B}|=8 \pi^{2} /(3 \sqrt{3})$. The operators $a_{\vec{x}, \sigma}^{ \pm}, b_{\vec{x}, \sigma}^{ \pm}$satisfy the canonical anticommutation rules and are periodic over $\Lambda$; their Fourier transforms are normalized in such a way that, if $\vec{k}, \vec{k}^{\prime}$ are both in the first Brillouin zone:

$$
\left\{\hat{a}_{\vec{k}, \sigma}^{\varepsilon}, \hat{a}_{\vec{k}^{\prime}, \sigma^{\prime}}^{\varepsilon^{\prime}}\right\}=L^{2} \delta_{\vec{k}, \vec{k}^{\prime}} \delta_{\varepsilon,-\varepsilon^{\prime}} \delta_{\sigma, \sigma^{\prime}}, \quad\left\{\hat{b}_{\vec{k}, \sigma}^{\varepsilon}, \hat{b}_{\vec{k}^{\prime}, \sigma^{\prime}}^{\varepsilon^{\prime}}\right\}=L^{2} \delta_{\vec{k}, \vec{k}^{\prime}} \delta_{\varepsilon,-\varepsilon^{\prime}} \delta_{\sigma, \sigma^{\prime}}
$$

Moreover, they are quasi-periodic over the first Brillouin zone:

$$
a_{\vec{k}+\vec{G}_{i}, \sigma}^{ \pm}=e^{ \pm i \vec{G}_{i} \cdot \vec{A}} a_{\vec{k}, \tau}^{ \pm}, \quad b_{\vec{k}+\vec{G}_{i}, \tau}^{ \pm}=e^{ \pm i \vec{G}_{i}\left(\vec{B}-\vec{\delta}_{j}\right)} b_{\vec{k}, \tau}^{ \pm}
$$

where $e^{i \vec{G}_{i} \vec{\delta}_{j}}=e^{i 2 \pi / 3}$, for all values of $i, j$. The phases $\vec{A}$ and $\vec{B}$ are arbitrary, the freedom in their choice corresponding to the freedom in the choice of the origins of the two sublattices $\Lambda_{A}$ and $\Lambda_{B}$ (this symmetry is sometimes referred to as Berry-gauge invariance). A convenient choice for $\vec{A}$ and $\vec{B}$, which makes the fields $\hat{a}_{\vec{k}, \sigma}^{ \pm}, \hat{b}_{\vec{k}, \sigma}^{ \pm}$periodic over the reciprocal lattice $\Lambda^{*}$, is $\vec{A}=\overrightarrow{0}$ and $\vec{B}=\vec{\delta}_{1}$, which reads:

$$
a_{\vec{x}, \sigma}^{ \pm}=L^{-2} \sum_{\vec{k} \in \mathcal{B}_{L}} e^{ \pm i \vec{k} \vec{x}} \hat{a}_{\vec{k}, \sigma}^{ \pm}, \quad b_{\vec{x}+\vec{\delta}_{1}, \sigma}^{ \pm}=L^{-2} \sum_{\vec{k} \in \mathcal{B}_{L}} e^{ \pm i \vec{k} \vec{x}} \hat{b}_{\vec{k}, \sigma}^{ \pm}, \quad \vec{x} \in \Lambda_{A} .
$$

This is the choice made in [11, 12], which will be used throughout this paper, too.

The grand-canonical Hamiltonian of the two-dimensional Hubbard model on the honeycomb lattice at half-filling is $H_{\Lambda}=H_{\Lambda}^{0}(t)+U V_{\Lambda}$, where $H_{\Lambda}^{0}(t)$ is the free Hamiltonian, describing nearest neighbor hopping $(t \in \mathbb{R}$ is the hopping parameter):

$$
H_{\Lambda}^{0}(t)=\left.H_{\Lambda}^{0}\left(\left\{t_{\vec{x}, j}\right\}\right)\right|_{t_{\vec{x}, j} \equiv t}, \quad H_{\Lambda}^{0}\left(\left\{t_{\vec{x}, j}\right\}\right)=-\sum_{\substack{\vec{x} \in \Lambda_{A} \\ j=1,2,3}} \sum_{\sigma=\uparrow \downarrow}\left(t_{\vec{x}, j} a_{\vec{x}, \sigma}^{+} b_{\vec{x}+\vec{\delta}_{j}, \sigma}^{-}+t_{\vec{x}, j}^{*} b_{\vec{x}+\vec{\delta}_{j}, \sigma}^{+} a_{\vec{x}, \sigma}^{-}\right),
$$

and $V_{\Lambda}$ is the local Hubbard interaction:

$$
V_{\Lambda}=\sum_{\substack{\vec{x} \in \Lambda_{A} \\ i=1,2,3}}\left(a_{\vec{x}, \uparrow}^{+} a_{\vec{x}, \uparrow}^{-}-\frac{1}{2}\right)\left(a_{\vec{x}, \downarrow}^{+} a_{\vec{x}, \downarrow}^{-}-\frac{1}{2}\right)+\sum_{\substack{\vec{x} \in \Lambda_{B} \\ i=1,2,3}}\left(b_{\vec{x}, \uparrow}^{+} b_{\vec{x}, \uparrow}^{-}-\frac{1}{2}\right)\left(b_{\vec{x}, \downarrow}^{+} b_{\vec{x}, \downarrow}^{-}-\frac{1}{2}\right)
$$


The current and density operators. The current is defined as usual via the Peierls substitution, by modifying the hopping parameter along the bond $\left(\vec{x}, \vec{x}+\vec{\delta}_{j}\right)$ as

$$
t \rightarrow t_{\vec{x}, j}(\vec{A})=t e^{i e \int_{0}^{1} \vec{A}\left(\vec{x}+s \vec{\delta}_{j}\right) \cdot \vec{\delta}_{j} d s},
$$

where the constant $e$ appearing at exponent is the electric charge and $\vec{A}(\vec{x}) \in \mathbb{R}^{2}$ is a periodic field on $\mathcal{S}_{\Lambda}=\left\{\vec{x}=L \xi_{1} \vec{l}_{1}+L \xi_{2} \vec{l}_{2}: \xi_{i} \in[0,1)\right\}$. Its Fourier transform is defined as $\vec{A}(\vec{x})=\left|\mathcal{S}_{\Lambda}\right|^{-1} \sum_{\vec{p} \in \mathcal{D}_{\Lambda}} \vec{A}_{\vec{p}} e^{-i \vec{p} \vec{x}}$, where $\left|\mathcal{S}_{\Lambda}\right|=\frac{3 \sqrt{3}}{2} L^{2}$ and $\mathcal{D}_{\Lambda}=\left\{\vec{p}=n_{1} \vec{G}_{1} / L+n_{2} \vec{G}_{2} / L: n_{i} \in\right.$ $\mathbb{Z}\}$; note that in the thermodynamic limit $\left|\mathcal{S}_{\Lambda}\right|^{-1} \sum_{\vec{p} \in \mathcal{D}_{\Lambda}} \rightarrow(2 \pi)^{-2} \int_{\mathbb{R}^{2}} d \vec{p}$. If we denote by $H_{\Lambda}(\vec{A})=H_{\Lambda}^{0}\left(\left\{t_{\vec{x}, j}(\vec{A})\right\}\right)+U V_{\Lambda}$ the modified Hamiltonian with the new hopping parameters, the lattice current is defined as $\vec{J}_{\vec{p}}^{(A)}=-\left|\mathcal{S}_{\Lambda}\right| \partial H_{\Lambda}(\vec{A}) / \partial \vec{A}_{\vec{p}}$, which gives, at first order in $\vec{A}$,

$$
\vec{J}_{\vec{p}}^{(A)}=\vec{J}_{\vec{p}}+\frac{1}{\left|\mathcal{S}_{\Lambda}\right|} \sum_{\vec{q} \in \mathcal{D}_{\Lambda}} \hat{\Delta}_{\vec{p}, \vec{q}} \vec{A}_{\vec{q}}
$$

where, if $\eta_{\vec{p}}^{j}=\frac{1-e^{-i \vec{p} \vec{d}_{j}}}{i \vec{p} \vec{p} j_{j}}$

$$
\begin{aligned}
\vec{J}_{\vec{p}} & =i e t \frac{1}{L^{2}} \sum_{\substack{\vec{k} \in \mathcal{B}_{L} \\
\sigma, j}} \vec{\delta}_{j} \eta_{\vec{p}}^{j}\left(\hat{a}_{\vec{k}+\vec{p}, \sigma}^{+} \hat{b}_{\vec{k}, \sigma}^{-} e^{-i \vec{k}\left(\vec{\delta}_{j}-\vec{\delta}_{1}\right)}-\hat{b}_{\vec{k}+\vec{p}, \sigma}^{+} \hat{a}_{\vec{k}, \sigma}^{-} e^{+i(\vec{k}+\vec{p})\left(\vec{\delta}_{j}-\vec{\delta}_{1}\right)}\right)= \\
& =\operatorname{iet} \sum_{\substack{\vec{x} \in \Lambda \\
\sigma, j}} e^{-i \vec{p} \vec{x}} \vec{\delta}_{j} \eta_{\vec{p}}^{j}\left(a_{\vec{x}, \sigma}^{+} b_{\vec{x}+\vec{\delta}_{j}, \sigma}^{-}-b_{\vec{x}+\vec{\delta}_{j}, \sigma}^{+} a_{\vec{x}, \sigma}^{-}\right)=: e v_{0} \sum_{\substack{\vec{x} \in \Lambda \\
j=1,2,3}} e^{-i \vec{p} \vec{x}} \vec{\delta}_{j} \eta_{\vec{p}}^{j} J_{\vec{x}}^{j}
\end{aligned}
$$

is the paramagnetic current (in the last rewriting, $v_{0}=3 t / 2$ is the free Fermi velocity and $J_{\vec{x}}^{j}=(2 i / 3)\left(a_{\vec{x}, \sigma}^{+} b_{\vec{x}+\vec{\delta}_{j}, \sigma}^{-}-b_{\vec{x}+\vec{\delta}_{j}, \sigma}^{+} a_{\vec{x}, \sigma}^{-}\right)$are the bond currents $)$and

$$
\left[\hat{\Delta}_{\vec{p}, \vec{q}}\right]_{l m}=\sum_{\substack{\vec{x} \in \Lambda \\ j=1,2,3}} e^{-i(\vec{p}+\vec{q}) \vec{x}}\left(\vec{\delta}_{j}\right)_{l}\left(\vec{\delta}_{j}\right)_{m} \eta_{\vec{p}}^{j} \eta_{\vec{q}}^{j} \Delta_{\vec{x}, j}
$$

with $\Delta_{\vec{x}, j}=-e^{2} t \sum_{\sigma}\left(a_{\vec{x}, \sigma}^{+} b_{\vec{x}+\vec{\delta}_{j}, \sigma}^{-}+b_{\vec{x}+\vec{\delta}_{j}, \sigma}^{+} a_{\vec{x}, \sigma}^{-}\right)$, is the diamagnetic tensor. Similarly, the density operator is defined by coupling the local density to an external field, i.e., by adding to the Hamiltonian a local chemical potential term of the form

$$
M_{\Lambda}(\mu)=-\sum_{\vec{x} \in \Lambda_{A}} \sum_{\sigma=\uparrow \downarrow} \mu(\vec{x}) a_{\vec{x}, \sigma}^{+} a_{\vec{x}, \sigma}^{-}-\sum_{\vec{x} \in \Lambda_{B}} \sum_{\sigma=\uparrow \downarrow} \mu(\vec{x}) b_{\vec{x}, \sigma}^{+} b_{\vec{x}, \sigma}^{-}
$$

where $\mu(\vec{x})=\left|\mathcal{S}_{\Lambda}\right|^{-1} \sum_{\vec{p} \in \mathcal{D}_{\Lambda}} \mu_{\vec{p}} e^{-i \vec{p} \vec{x}}$ is a periodic field on $\mathcal{S}_{\Lambda}$. If we denote by $H_{\Lambda}(\mu)=$ $H_{\Lambda}^{0}(t)+U V_{\Lambda}+M_{\Lambda}(\mu)$ the modified Hamiltonian in the presence of the local chemical po- 
tential, the lattice density is defined as $\hat{\rho}_{\vec{p}}=-\left|\mathcal{S}_{\Lambda}\right| \partial H_{\Lambda}(\mu) / \partial \mu_{\vec{p}}$, which gives

$$
\begin{aligned}
\hat{\rho}_{\vec{p}} & =\frac{1}{L^{2}} \sum_{\substack{\vec{k} \in \mathcal{B}_{L} \\
\sigma=\uparrow \downarrow}}\left(\hat{a}_{\vec{k}+\vec{p}, \sigma}^{+} \hat{a}_{\vec{k}, \sigma}^{-}+e^{-i \vec{p} \hat{\delta}_{1}} \hat{b}_{\vec{k}+\vec{p}, \sigma}^{+} \hat{b}_{\vec{k}, \sigma}^{-}\right)= \\
& =\sum_{\substack{\vec{x} \in \Lambda_{A} \\
\sigma=\uparrow \downarrow}} e^{-i \vec{p} \vec{x}} a_{\vec{x}, \sigma}^{+} a_{\vec{x}, \sigma}^{-}+\sum_{\substack{\vec{x} \in \Lambda_{B} \\
\sigma=\uparrow \downarrow}} e^{-i \vec{p} \vec{x}} b_{\vec{x}, \sigma}^{+} b_{\vec{x}, \sigma}^{-}=: \sum_{\vec{x} \in \Lambda_{A}} e^{-i \vec{p} \vec{x}} \rho_{\vec{x}}^{A}+\sum_{\vec{x} \in \Lambda_{B}} e^{-i \vec{p} \vec{x}} \rho_{\vec{x}}^{B} .
\end{aligned}
$$

It will be convenient for the incoming discussion to think the two components of the paramagnetic current $\hat{J}_{\vec{p}, l}, l=1,2$, as the spatial components of a "space-time" three-components vector $\hat{J}_{\vec{p}, \mu}, \mu=0,1,2$, with $\hat{J}_{\vec{p}, 0}=e \hat{\rho}_{\vec{p}}$. In the following, it will also convenient to introduce the reduced current $\overrightarrow{\vec{p}}$, related to the paramagnetic current by

$$
\vec{J}_{\vec{p}}=v_{0} \vec{\jmath}_{\vec{p}}
$$

with $v_{0}=3 t / 2$ the free Fermi velocity.

Schwinger functions and response functions. The thermal state of the system at inverse temperature $\beta>0$, associated to the density matrix $e^{-\beta H_{\Lambda}}$, can be characterized in terms of Schwinger functions and response functions. The Schwinger functions are the analytic continuation to imaginary time of the off-diagonal elements of the reduced density matrices; the response functions give us informations about the reaction of the system to a diversity of external probes within the linear response regime. They are defined as follows. Let $\psi_{\vec{x}, \sigma}^{ \pm}=\left(a_{\vec{x}, \sigma}^{ \pm}, b_{\vec{x}+\vec{\delta}_{1}, \sigma}^{ \pm}\right)$, let $O_{\vec{x}_{i}}^{(i)}, i=1, \ldots, n$, be local monomials in the $\psi_{\vec{x}, \sigma}^{ \pm}$operators and let us denote by $O_{\mathbf{x}_{i}}^{(i)}=e^{x_{i, 0} H_{\Lambda}} O_{\vec{x}_{i}}^{(i)} e^{-x_{i, 0} H_{\Lambda}}$ the corresponding imaginary-time evolved operators; here $\mathbf{x}_{i}=\left(x_{i, 0}, \vec{x}_{i}\right)$ and $x_{i, 0} \in[0, \beta)$ is the imaginary time. The average of a product of local operators in the thermal state of the system at inverse temperature $\beta>0$ is defined as

$$
\left\langle O_{\mathbf{x}_{1}}^{(1)} \cdots O_{\mathbf{x}_{n}}^{(n)}\right\rangle_{\beta, L}=\frac{\operatorname{Tr}\left\{e^{-\beta H_{\Lambda}} \mathbf{T}\left(O_{\mathbf{x}_{1}}^{(1)} \cdots O_{\mathbf{x}_{n}}^{(n)}\right)\right\}}{\operatorname{Tr}\left\{e^{e^{-\beta H_{\Lambda}}}\right\}},
$$

where $\mathbf{T}$ is the operator of fermionic time ordering, acting on a product of fermionic fields as:

$$
\mathbf{T}\left(\psi_{\mathbf{x}_{1}, \sigma_{1}, \rho_{1}}^{\varepsilon_{1}} \cdots \psi_{\mathbf{x}_{n}, \sigma_{n}, \rho_{n}}^{\varepsilon_{n}}\right)=(-1)^{\pi} \psi_{\mathbf{x}_{\pi(1)}, \sigma_{\pi(1)}, \rho_{\pi(1)}}^{\varepsilon_{\pi(1)}} \cdots \psi_{\mathbf{x}_{\pi(n)}, \sigma_{\pi(n)}, \rho_{\pi(n)}}^{\varepsilon_{\pi(n)}}
$$

where $\varepsilon_{i} \in\{+,-\}, \sigma_{i} \in\{\uparrow, \downarrow\}, \rho_{i} \in\{1,2\}, \mathbf{x}_{i} \in[0, \beta) \times \Lambda, \psi_{\mathbf{x}, \sigma, 1}^{ \pm}=a_{\mathbf{x}, \sigma}^{ \pm}$and, if $\boldsymbol{\delta}_{i}=\left(0, \vec{\delta}_{i}\right)$, $\psi_{\mathbf{x}, \sigma, 2}^{ \pm}=b_{\mathbf{x}+\boldsymbol{\delta}_{1}, \sigma}^{ \pm}$. Moreover, $\pi$ is a permutation of $\{1, \ldots, n\}$, chosen in such a way that $x_{\pi(1) 0} \geq \cdots \geq x_{\pi(n) 0}$, and $(-1)^{\pi}$ is its sign. [If some of the time coordinates are equal each 
other, the arbitrariness of the definition is solved by ordering each set of operators with the same time coordinate so that creation operators precede the annihilation operators.] Finally, we denote by $\left\langle O_{\mathbf{x}_{1}}^{(1)} ; \cdots ; O_{\mathbf{x}_{n}}^{(n)}\right\rangle_{\beta, L}$ the corresponding truncated expectations. We shall also use the notation $\langle\cdot\rangle_{\beta}=\lim _{L \rightarrow \infty}\langle\cdot\rangle_{\beta, L}$ and $\langle\cdot\rangle=\lim _{L \rightarrow \infty}\langle\cdot\rangle_{\beta}$.

Choosing the local operators $O_{\mathbf{x}_{i}}^{(i)}$ in Eq.(1.16) simply as monomials in the fermionic fields, we get the Schwinger functions of order $n$ :

$$
S_{n}^{\beta, L}\left(\mathbf{x}_{1}, \varepsilon_{1}, \sigma_{1} ; \ldots ; \mathbf{x}_{n}, \varepsilon_{n}, \sigma_{n}\right)_{\rho_{1}, \ldots, \rho_{n}}=\left\langle\psi_{\mathbf{x}_{1}, \sigma_{1}, \rho_{1}}^{\varepsilon_{1}} \cdots \psi_{\mathbf{x}_{n}, \sigma_{n}, \rho_{n}}^{\varepsilon_{n}}\right\rangle_{\beta, L} .
$$

Choosing the operators $O_{\mathbf{x}_{i}}^{(i)}$ as suitable combinations of the current and density operators, we get the current-current, density-density and current-density response functions:

$$
\hat{K}_{\mu \nu}^{\beta, L}(\mathbf{p}):=\frac{1}{\beta L^{2}} \int_{-\beta / 2}^{\beta / 2} d x_{0} \int_{-\beta / 2}^{\beta / 2} d y_{0} e^{-i p_{0}\left(x_{0}-y_{0}\right)}\left\langle J_{\left(x_{0}, \vec{p}\right), \mu} ; J_{\left(y_{0},-\vec{p}\right), \nu}\right\rangle_{\beta, L},
$$

where $\mathbf{p}=\left(p_{0}, \vec{p}\right) \in \frac{2 \pi}{\beta} \mathbb{Z} \times \mathcal{D}_{\Lambda}$ and $J_{\left(x_{0}, \vec{p}\right), \mu}=e^{x_{0} H_{\Lambda}} \hat{J}_{\vec{p}, \mu} e^{-x_{0} H_{\Lambda}}$. An important role will be also played in the following by the two- and three-points functions:

$$
\begin{gathered}
\hat{S}^{\beta, L}(\mathbf{k}):=\frac{1}{\beta L^{2}} \int_{(\beta, L)} d \mathbf{x} \int_{(\beta, L)} d \mathbf{y} e^{+i \mathbf{k}(\mathbf{x}-\mathbf{y})} S_{2}^{\beta, L}(\mathbf{x},-, \sigma ; \mathbf{y},+, \sigma), \\
\hat{G}_{2,1 ; \mu}^{\beta, L}(\mathbf{k}, \mathbf{p}):=\frac{1}{\beta L^{2}} \int_{-\beta / 2}^{\beta / 2} d x_{0} \int_{-\beta / 2}^{\beta / 2} d y_{0} \int_{-\beta / 2}^{\beta / 2} d z_{0} e^{+i k_{0}\left(x_{0}-y_{0}\right)+i p_{0}\left(x_{0}-z_{0}\right)}\left\langle J_{\left(z_{0}, \vec{p}\right), \mu} ; \psi_{\left(x_{0}, \vec{k}+\vec{p}\right), \sigma}^{-} \psi_{\left(y_{0}, \vec{k}\right), \sigma}^{+}\right\rangle_{\beta, L}
\end{gathered}
$$

where $\int_{(\beta, L)} d \mathbf{x}$ is a shorthand for $\int_{-\beta / 2}^{\beta / 2} d x_{0} \sum_{\vec{x} \in \Lambda}$ and $\psi_{\left(x_{0}, \vec{k}\right), \sigma}^{ \pm}=e^{x_{0} H_{\Lambda}} \hat{\psi}_{\vec{k}, \sigma} e^{-x_{0} H_{\Lambda}}$, with $\hat{\psi}_{\vec{k}, \sigma}^{ \pm}=\left(\hat{a}_{\vec{k}, \sigma}^{ \pm}, \hat{b}_{\vec{k}, \sigma}^{ \pm}\right)$. Here and in the following, we will exploit whenever possible the vectorial structure of $\psi$ and the tensorial structure of products of $\psi$ 's; to this purpose, we will think of $\psi^{-}$as a column vector and $\psi^{+}$as a row vector, so that, e.g., $\psi_{\mathbf{x}, \sigma}^{+} \psi_{\mathbf{y}, \sigma}^{-}$will be naturally thought as a scalar, while $\psi_{\mathbf{x}, \sigma}^{-} \psi_{\mathbf{y}, \sigma}^{+}$will be thought as a $2 \times 2$ matrix. In particular, both $\hat{S}^{\beta, L}(\mathbf{k})$ and $\hat{G}_{2,1 ; \mu}^{\beta, L}(\mathbf{k}, \mathbf{p})$ (for any fixed choice of $\mu \in\{0,1,2\}$ ) can be thought as $2 \times 2$ matrices.

Conductivity. The ac conductivity in units such that $\hbar=1$ is related to the current-current correlations via Kubo formula [7], which reads, for all $2 \pi \beta^{-1} \mathbb{Z} \ni p_{0} \neq 0$ and $l, m \in\{1,2\}$ :

$$
\sigma_{l m}^{\beta}\left(p_{0}\right)=-\frac{2}{3 \sqrt{3}} \frac{1}{p_{0}}\left[\hat{K}_{l m}^{\beta}\left(p_{0}, 0\right)+\Delta_{l m}^{\beta}\right]
$$

where $\hat{K}_{l m}^{\beta}(\mathbf{p})=\lim _{L \rightarrow+\infty} \hat{K}_{l m}^{\beta, L}(\mathbf{p})$ and

$$
\Delta_{l m}^{\beta}=\lim _{L \rightarrow \infty} \frac{1}{L^{2}} \sum_{\substack{\vec{x} \in \Lambda \\ j=1,2,3}}\left(\vec{\delta}_{j}\right)_{l}\left(\vec{\delta}_{j}\right)_{m}\left\langle\Delta_{\vec{x}, j}\right\rangle_{\beta, L}
$$


is the diamagnetic contribution (see Eq.(1,12) ) and the factor $2 /(3 \sqrt{3})$ appearing in Eq.(1,22) must be understood as the inverse of the area of the unit cell of the hexagonal lattice.

The main goal of this paper is to compute $\sigma_{l m}^{\beta}\left(p_{0}\right)$ in the zero temperature and zero frequency limit (taking the limits in a suitable order, so to make contact with experiments on the optical conductivity of graphene), i.e., to compute the so-called universal optical conductivity:

$$
\sigma_{l m}:=\lim _{p_{0} \rightarrow 0^{+}} \lim _{\beta \rightarrow \infty} \sigma_{l m}^{\beta}\left(p_{0}\right) .
$$

A key role in its computation will be played by Ward Identities, which show that the quantities introduced above are not independent; on the contrary, they are related by exact identities, which we now describe.

\section{B. Conservation laws and Ward Identities}

By definition of $\rho_{\left(x_{0}, \vec{p}\right)}=e^{x_{0} H_{\Lambda}} \hat{\rho}_{\vec{p}} e^{-x_{0} H_{\Lambda}}$, we have:

$$
\partial_{x_{0}} \rho_{\left(x_{0}, \vec{p}\right)}=\left[H_{\Lambda}, \rho_{\left(x_{0}, \vec{p}\right)}\right]
$$

Computing explicitly the r.h.s. of this equation and using, in particular, the fact that $\left[V_{\Lambda}, \rho_{\vec{p}}\right]=0$, we find:

$$
\partial_{x_{0}} \rho_{\left(x_{0}, \vec{p}\right)}=t \sum_{\substack{\vec{x} \in \Lambda \\ \sigma, j}} e^{-i \vec{p} \vec{x}}\left(1-e^{-i \vec{p} \vec{\phi}_{j}}\right)\left(a_{\mathbf{x}, \sigma}^{+} b_{\mathbf{x}+\boldsymbol{\delta}_{j}, \sigma}^{-}-b_{\mathbf{x}+\boldsymbol{\delta}_{j}, \sigma}^{+} a_{\mathbf{x}, \sigma}^{-}\right)
$$

Comparing the r.h.s. of this equation with the definition of the paramagnetic current, we recognize that Eq.(1,26) can be rewritten as a continuity equation:

$$
-i e \partial_{x_{0}} \rho_{\left(x_{0}, \vec{p}\right)}+i \vec{p} \cdot \vec{J}_{\left(x_{0}, \vec{p}\right)}=0
$$

Using the continuity equation Eq.(11,27), we can easily derive an exact identity relating three- and two-point functions. In fact, by the definition of $\hat{G}_{2,1 ; 0}^{\beta, L}(\mathbf{k}, \mathbf{p})$, see Eq.(1.21), and integrating by parts, we find:

$$
i p_{0} \hat{G}_{2,1 ; 0}^{\beta, L}(\mathbf{k}, \mathbf{p})=\frac{1}{L^{2}} \int_{-\beta / 2}^{\beta / 2} d y_{0} \int_{-\beta / 2}^{\beta / 2} d z_{0} e^{-i k_{0} y_{0}-i p_{0} z_{0}} \partial_{z_{0}}\left\langle J_{\left(z_{0}, \vec{p}\right), 0} ; \psi_{(0, \vec{k}+\vec{p}), \sigma}^{-} \psi_{\left(y_{0}, \vec{k}\right), \sigma}^{+}\right\rangle_{\beta, L}
$$


The derivative with respect to $z_{0}$ can act either on $J_{\left(z_{0}, \vec{p}\right), 0}$, in which case we use the continuity equation, or on the Heaviside step functions involved in the definition of time-ordering operator T. After some straightforward algebra, and using the fact that

$$
\left[\hat{\rho}_{\vec{p}}, \hat{\psi}_{\vec{k}+\vec{p}, \sigma}^{-}\right]=-M(\vec{p}) \hat{\psi}_{\vec{k}, \sigma}^{-}, \quad\left[\hat{\rho}_{\vec{p}}, \hat{\psi}_{\vec{k}, \sigma}^{+}\right]=\psi_{\vec{k}+\vec{p}, \sigma}^{+} M(\vec{p})
$$

with

$$
M(\vec{p})=\left(\begin{array}{cc}
1 & 0 \\
0 & e^{-i \vec{p} \vec{p}_{1}}
\end{array}\right)
$$

we end up with

$$
-i p_{0} \hat{G}_{2,1 ; 0}^{\beta, L}(\mathbf{k}, \mathbf{p})+p_{1} \hat{G}_{2,1 ; 1}^{\beta, L}(\mathbf{k}, \mathbf{p})+p_{2} \hat{G}_{2,1 ; 2}^{\beta, L}(\mathbf{k}, \mathbf{p})=-e \hat{S}^{\beta, L}(\mathbf{k}+\mathbf{p}) M(\vec{p})+e M(\vec{p}) \hat{S}^{\beta, L}(\mathbf{k}) .
$$

Proceeding in the same way, we also find:

$$
\begin{aligned}
& -i p_{0} \hat{K}_{0,0}^{\beta, L}(\mathbf{p})+p_{1} \hat{K}_{1,0}^{\beta, L}(\mathbf{p})+p_{2} \hat{K}_{2,0}^{\beta, L}(\mathbf{p})=0 \\
& -i p_{0} \hat{K}_{0, m}^{\beta, L}(\mathbf{p})+p_{1} \hat{K}_{1, m}^{\beta, L}(\mathbf{p})+p_{2} \hat{K}_{2, m}^{\beta, L}(\mathbf{p})=-\frac{1}{L^{2}}\left[\vec{p} \cdot\left\langle\hat{\Delta}_{\vec{p},-\vec{p}}\right\rangle_{\beta, L}\right]_{m},
\end{aligned}
$$

where $m=1,2$, and we used the fact that

$$
e\left[\hat{\rho}_{\vec{p}}, \vec{J}_{-\vec{p}}\right]=\sum_{\substack{\vec{x} \in \Lambda \\ j=1,2,3}}\left(\vec{p} \cdot \vec{\delta}_{j}\right) \vec{\delta}_{j}\left|\eta_{\vec{p}}^{j}\right|^{2} \Delta_{\vec{x}, j}=\vec{p} \cdot \hat{\Delta}_{\vec{p},-\vec{p}} .
$$

The term in the r.h.s. of Eq.(11.33) is known as Schwinger term.

Note that from Eq.(11.33), setting, e.g., $p_{2}=0$, we find that, for $i=1,2$,

$$
\hat{K}_{1 i}\left(p_{0}, p_{1}, 0\right)+\lim _{\beta, L \rightarrow \infty} \frac{1}{L^{2}}\left\langle\left[\hat{\Delta}_{\left(p_{1}, 0\right),\left(-p_{1}, 0\right)}\right]_{1 i}\right\rangle_{\beta, L}=i \frac{p_{0}}{p_{1}} \hat{K}_{0 i}\left(p_{0}, p_{1}, 0\right),
$$

provided the limits of these functions as $\beta, L \rightarrow \infty$ exist.

Now, if we knew that the correlations in Eq.(11.35) were continuous and continuosly differentiable at $\mathbf{p}=\mathbf{0}$, from the latter equation we would conclude that $\lim _{\mathbf{p} \rightarrow \mathbf{0}}\left[\hat{K}_{1 i}(\mathbf{p})+\Delta_{1 i}\right]=0$ (with $\Delta_{l m}=\lim _{\beta \rightarrow \infty} \Delta_{l m}^{\beta}$, see Eq.(11.23) ); moreover, continuous differentiability together with the symmetry properties of our model would imply that (with a proof analogous to the one of item (ii) in Proposition 1 below) $\lim _{\mathbf{p} \rightarrow \mathbf{0}} \partial_{p_{0}} \hat{K}_{1 i}(\mathbf{p})=0$. Comparing these equations with the definition of the universal optical conductivity, see Eqs.(11.22)-(11.24), then we would be tempted to conclude that $\sigma_{1 i}=0$ (and a similar argument would imply that $\sigma_{2 i}=0$ ). This is in contrast with the explicit computation of the conductivity in the non-interacting 
case, which will be discussed below. The solution to this apparent paradox is that $\hat{K}_{l m}(\mathbf{p})$ is not continuosly differentiable at $\mathbf{p}=\mathbf{0}$. In fact, as it will turn out from the following discussion, the regularity properties of the Fourier transform of the current-current correlations play a crucial role in the physical properties of the conductivity.

\section{Properties of the non interacting model}

In the absence of interactions, that is for $U=0$, the two-points function defined in Eq.(11.20) reads (see [11, 12]):

$$
\left.\hat{S}^{\beta, L}(\mathbf{k})\right|_{U=0}=\frac{1}{k_{0}^{2}+v_{0}^{2}|\Omega(\vec{k})|^{2}}\left(\begin{array}{cc}
i k_{0} & -v_{0} \Omega^{*}(\vec{k}) \\
-v_{0} \Omega(\vec{k}) & i k_{0}
\end{array}\right)=: S_{0}(\mathbf{k}),
$$

where $v_{0}=\frac{3}{2} t$ and $\Omega(\vec{k})=\frac{2}{3} \sum_{j=1}^{3} e^{i \vec{k}\left(\vec{\delta}_{j}-\vec{\delta}_{1}\right)}$. The complex dispersion relation $\Omega(\vec{k})$ vanishes only at the two Fermi points

$$
\vec{p}_{F}^{ \pm}=\left(\frac{2 \pi}{3}, \pm \frac{2 \pi}{3 \sqrt{3}}\right)
$$

close to which it behaves as follows: $\Omega\left(\vec{p}_{F}^{ \pm}+\vec{k}^{\prime}\right)=i k_{1}^{\prime} \pm k_{2}^{\prime}+O\left(\left|\vec{k}^{\prime}\right|^{2}\right)$. The Schwinger functions of higher order can be explictly computed in terms of $S_{0}(\mathbf{k})$ via the fermionic Wick rule.

Also the universal optical conductivity can be computed explicitly, see Appendix $\mathrm{A}$, and turns out to be equal to (restoring the presence of the dimensional constant $\hbar=h /(2 \pi)$ in the result):

$$
\left.\sigma_{i j}\right|_{U=0}=\frac{2}{3 \sqrt{3}} \frac{2 e^{2} v_{0}^{2}}{\hbar} \lim _{p_{0} \rightarrow 0^{+}} \int \frac{d k_{0}}{2 \pi} \int_{\mathcal{B}} \frac{d \vec{k}}{|\mathcal{B}|} \operatorname{Tr}\left\{\frac{S_{0}\left(\mathbf{k}+\left(p_{0}, \overrightarrow{0}\right)\right)-S_{0}(\mathbf{k})}{p_{0}} \Gamma_{i}(\vec{k}, \overrightarrow{0}) S_{0}(\mathbf{k}) \Gamma_{j}(\vec{k}, \overrightarrow{0})\right\},
$$

where

$$
\vec{\Gamma}(\vec{k}, \vec{p})=\frac{2}{3} \sum_{j=1}^{3} \vec{\delta}_{j}\left(\begin{array}{cc}
0 & i e^{-i \vec{k}\left(\vec{\delta}_{j}-\vec{\delta}_{1}\right)} \\
-i e^{+i(\vec{k}+\vec{p})\left(\vec{\delta}_{j}-\vec{\delta}_{1}\right)} & 0
\end{array}\right)
$$

Note that the r.h.s. of Eq.(11.38) depends on $v_{0}$. Moreover, the integral is not uniformly convergent in $p_{0}$ as $p_{0} \rightarrow 0$; in particular, it is well known that one cannot exchange the limit with the integral [13]. An explicit computation, see Appendix [A, yields

$$
\left.\sigma_{i j}\right|_{U=0}=\frac{e^{2}}{h} \frac{\pi}{2} \delta_{i j}
$$

a value that, remarkably, does not depend on $v_{0}$. It is also remarkable that the same value of the conductivity is found in the so-called Dirac approximation, that is by replacing $S_{0}(\mathbf{k})$ 
in Eq.(1,40) with its linear approximation around the Fermi points, in the presence of an ultraviolet cutoff, i.e.,

$\sum_{\omega= \pm} \chi\left(\left|\vec{k}-\vec{p}_{F}^{\omega}\right| \leq \varepsilon\right) \hat{G}_{\omega}^{v_{0}}\left(k_{0}, \vec{k}-\vec{p}_{F}^{\omega}\right), \quad \hat{G}_{\omega}^{v_{0}}(\mathbf{k})=\left(\begin{array}{cc}-i k_{0} & -v_{0}\left(-i k_{1}+\omega k_{2}\right) \\ -v_{0}\left(i k_{1}+\omega k_{2}\right) & -i k_{0}\end{array}\right)^{-1}$,

where $\varepsilon$ is a small positive number. Therefore, $\left.\sigma_{i j}\right|_{U=0}$ does not depend on the lattice parameters and, in this sense, we can say that the free conductivity is universal.

\section{Universality of the conductivity}

Let us now discuss the effects of the Hubbard interaction. We will first recall the results of [11, 12], concerning the thermodynamic functions of the model, the Schwinger functions and, in particular, the two- and three-points functions. Next, we will state our results concerning the interacting conductivity.

In Theorem 1 of [11], we proved that, if $U$ is small enough (uniformly in the system size and in the temperature), then the specific free energy $f_{\beta}(U)$ and the finite temperature Schwinger functions in the thermodynamic limit are analytic functions of $U$, uniformly in $\beta$ as $\beta \rightarrow \infty$, and so are the specific ground state energy $e(U)$ and the zero temperature Schwinger functions in the thermodynamic limit. In particular, we proved that the Fourier transform of the zero-temperature two-points function in the thermodynamic limit is singular only at the Fermi points $\mathbf{k}=\mathbf{p}_{F}^{ \pm}=\left(0, \vec{p}_{F}^{ \pm}\right)$and, close to the singularities, if $\omega= \pm$, it can be written as

$$
\hat{S}\left(\mathbf{p}_{F}^{\omega}+\mathbf{k}^{\prime}\right)=\frac{1}{Z}\left(\begin{array}{cc}
-i k_{0} & -v_{F} \Omega^{*}\left(\vec{p}_{F}^{\omega}+\vec{k}^{\prime}\right) \\
-v_{F} \Omega\left(\vec{p}_{F}^{\omega}+\vec{k}^{\prime}\right) & -i k_{0}
\end{array}\right)^{-1}\left(\mathbb{1}+R\left(\mathbf{k}^{\prime}\right)\right)
$$

where $Z$ and $v_{F}$ are two analytic functions of $U$, analytically close to their unperturbed values,

$$
Z=1+O\left(U^{2}\right), \quad v_{F}=v_{0}+O\left(U^{2}\right)
$$

Moreover the matrix $R\left(\mathbf{k}^{\prime}\right)$ satisfies $\left\|R\left(\mathbf{k}^{\prime}\right)\right\| \leq C\left|\mathbf{k}^{\prime}\right|^{\vartheta}$ for some constants $C, \vartheta>0$ and $\left|\mathbf{k}^{\prime}\right|$ small enough. In [12] we also announced the following result, whose detailed proof will be given below. 
Theorem 2. There exists $U_{0}>0$ such that, the three-point function Eq.(1.21) is uniformly analytic in $\beta, L$ and uniformly convergent to an analytic function as $\beta, L \rightarrow \infty$, for all $\mathbf{k} \neq \mathbf{p}_{F}^{ \pm}, \mathbf{p} \neq \mathbf{0}$ and for $\left(\min _{\omega}\left|\mathbf{k}-\mathbf{p}_{F}^{\omega}\right|\right),|\mathbf{p}| \cdot\left(\min _{\omega}\left|\mathbf{k}-\mathbf{p}_{F}^{\omega}\right|\right)^{-1}$ sufficiently small. Moreover, the vertex function at the Fermi points (in the thermodynamic and zero-temperature limits) is equal to:

$$
\lim _{\mathbf{k} \rightarrow \mathbf{p}_{F}^{\omega}} \lim _{\mathbf{p} \rightarrow \mathbf{0}}[\hat{S}(\mathbf{k}+\mathbf{p})]^{-1} \hat{G}_{2,1 ; \mu}(\mathbf{k}, \mathbf{p})[\hat{S}(\mathbf{k})]^{-1}=e Z_{\mu} \Gamma_{\mu}\left(\vec{p}_{F}^{\omega}, \overrightarrow{0}\right)
$$

where $\Gamma_{i}(\vec{k}, \vec{p}), i=1,2$, were defined in Eq.(11.39), $\Gamma_{0}(\vec{k}, \vec{p})=1$ and $Z_{\mu}=Z_{\mu}(U), \mu=0,1,2$, are analytic functions of $U$.

Note that the existence of the limits in Eq.(11,44) is part of the statement of the theorem. An important consequence of Theorem 2 (also announced in [12]) is obtained by combining its result with the WI Eq.(11,31): in fact, using Eqs.(1,42)-(1,44) into Eq.(11.31) we find that the vertex functions are related to the wave function renormalization $Z$ and to the Fermi velocity $v_{F}$ by simple identities:

$$
Z_{0}=Z, \quad Z_{1}=Z_{2}=v_{F} Z
$$

These relations can be proven as follows: take the limits in the 1.h.s. of Eq.(1.44) with $\mathbf{k}=\mathbf{p}_{F}^{\omega}+k^{\prime} \mathbf{e}_{\mu}$ and $\mathbf{p}=p^{\prime} \mathbf{e}_{\mu}$, where $\mathbf{e}_{0}=(1,0,0), \mathbf{e}_{1}=(0,1,0)$ and $\mathbf{e}_{2}=(0,0,1) ;$ using Eq.(1131) and Eq.(11,42) we can rewrite the 1.h.s. of Eq.(1144) (after having taken $p^{\prime} \rightarrow 0$ ) as:

$$
\begin{aligned}
& -e(i)^{\delta_{\mu, 0}} \lim _{k^{\prime} \rightarrow 0}\left[\hat{S}\left(\mathbf{p}_{F}^{\omega}+k^{\prime} \mathbf{e}_{\mu}\right)\right]^{-1} \partial_{\mu} \hat{S}\left(\mathbf{p}_{F}^{\omega}+k^{\prime} \mathbf{e}_{\mu}\right)\left[\hat{S}\left(\mathbf{p}_{F}^{\omega}+k^{\prime} \mathbf{e}_{\mu}\right)\right]^{-1}= \\
& =e(i)^{\delta_{\mu, 0}} \lim _{k^{\prime} \rightarrow 0} \partial_{\mu}\left[\hat{S}\left(\mathbf{p}_{F}^{\omega}+k^{\prime} \mathbf{e}_{\mu}\right)\right]^{-1}
\end{aligned}
$$

that, using again Eq.(1.42), is equal to $e Z\left(v_{F}\right)^{1-\delta_{\mu, 0}} \Gamma_{\mu}\left(\vec{p}_{F}^{\omega}, \overrightarrow{0}\right)$.

To summarize, at half filling, weak local electron-electron interactions do not change the infrared behavior of correlations: they "just" change the values of some physical parameters, namely the wave function renormalization $Z$, the Fermi velocity $v_{F}$ and the vertex functions $Z_{\mu}$; the latter are related to $Z$ and $v_{F}$ in a simple way, thanks to WIs. The next natural question we would like to answer to is how is the conductivity changed by the presence of interactions. Since the infrared behavior of the interacting correlations is the same as 
the non-interacting one, it is natural to expect that the interacting conductivity remains finite in the zero-temperature and zero-frequency limit; what is apriori unclear is whether the zero-frequency conductivity remains universal in any reasonable sense, in analogy with the universal behavior of the free conductivity. Quite remarkably, we can prove that the interacting conductivity is universal in a very strong sense: namely, we prove that $\sigma_{l m}$ is not only independent of the details of the lattice, but it is also exactly independent of $U$.

Theorem 3. There exists a constant $U_{0}>0$ such that, for $|U| \leq U_{0}$ and any fixed $p_{0}$ (non vanishing and sufficiently small), $\sigma_{l m}^{\beta}\left(p_{0}\right)$ is analytic in $U$ uniformly in $\beta$ as $\beta \rightarrow \infty$ and uniformly convergent to an analytic function of $U$ as $\beta \rightarrow \infty$. Moreover,

$$
\sigma_{l m}=\lim _{p_{0} \rightarrow 0^{+}} \lim _{\beta \rightarrow \infty} \sigma_{l m}^{\beta}\left(p_{0}\right)=\frac{e^{2}}{h} \frac{\pi}{2} \delta_{l m} .
$$

Note that the limit $\beta \rightarrow \infty$ is taken before the limit $p_{0} \rightarrow 0^{+}$. In other words, the theorem says that the interaction corrections to the conductivity are negligible at frequencies $\beta^{-1} \ll p_{0} \ll t$, in agreement with experiments on the optical conductivity [5]. The above results says that all the interaction corrections to the conductivity cancel out exactly, even if the Fermi velocity and the wave function are renormalized by the interaction.

The proof of Theorem 3 is based on two main ingredients: (i) the use of the exact Ward Identities Eqs.(11.32)-(11.33); (ii) the fact that the interaction-dependent corrections to the Fourier transform of the current-current correlations are differentiable with continuous derivative (in contrast, the free part is continuous and not differentiable at zero frequency). The main technical point of this paper is to control the regularity properties of the interaction corrections to the conductivity, which are summarized in the following proposition.

Proposition 1. There exists $U_{0}>0$ such that, if $|U| \leq U_{0}$, then the current-current function $\hat{K}_{l m}^{\beta, L}(\mathbf{p})$ is analytic in $U$, uniformly in $\beta, L$, for all sufficiently small $\mathbf{p} \neq \mathbf{0}$ and it is uniformly convergent as $\beta, L$ to the function $\hat{K}_{l m}(\mathbf{p})=\lim _{\beta \rightarrow \infty} \lim _{L \rightarrow \infty} \hat{K}_{l m}^{\beta, L}(\mathbf{p})$, which is also analytic in $U$ for all sufficiently $\mathbf{p} \neq \mathbf{0}$. The latter function satisfies the following properties:

1. $\hat{K}_{l m}(\mathbf{p})$ is continuous for all sufficiently small $\mathbf{p} \in \mathbb{R} \times \mathcal{B}$ (in particular at $\mathbf{p}=\mathbf{0}$ ) and continuously differentiable for all sufficiently small $\mathbf{p} \neq \mathbf{0}$. 
2. $\hat{K}_{l m}(\mathbf{p})$ can be decomposed as:

$$
\hat{K}_{l m}(\mathbf{p})=\frac{Z_{l} Z_{m}}{Z^{2}}\left\langle\hat{\jmath}_{\mathbf{p}, l} ; \hat{\jmath}_{-\mathbf{p}, m}\right\rangle^{0, v_{F}}+R_{\mu \nu}(\mathbf{p}),
$$

where:

(i) if $\langle\cdot\rangle_{\beta, L}^{0, v_{F}}$ the average with respect to the density matrix $e^{-\beta H_{\Lambda}^{0}\left(\frac{2}{3} v_{F}\right)}$ associated to the non-interacting Hamiltonian with Fermi velocity $v_{F}$, then

$$
\left\langle\hat{\jmath}_{\mathbf{p}, l} ; \hat{\jmath}_{-\mathbf{p}, m}\right\rangle^{0, v_{F}}=\lim _{\beta, L \rightarrow \infty} \frac{1}{\beta L^{2}} \int_{-\beta / 2}^{\beta / 2} d x_{0} \int_{-\beta / 2}^{\beta / 2} d y_{0} e^{-i p_{0}\left(x_{0}-y_{0}\right)}\left\langle j_{\left(x_{0}, \vec{p}\right), l} ; j_{\left(y_{0},-\vec{p}\right), m}\right\rangle_{\beta, L}^{0, v_{F}},
$$

with $j_{\left(x_{0}, \vec{p}\right), l}=e^{x_{0} H_{\Lambda}} j_{\vec{p}, l} e^{-x_{0} H_{\Lambda}}$ and $\vec{\jmath}_{\vec{p}}$ the reduced current defined in Eq. (11.15);

(ii) $R(\mathbf{p})$ is continuously differentiable for all sufficiently small $\mathbf{p} \in \mathbb{R} \times \mathcal{B}$ (in particular at $\mathbf{p}=\mathbf{0})$; moreover, $R\left(p_{0}, \overrightarrow{0}\right)=R\left(-p_{0}, \overrightarrow{0}\right)$.

One can immediately realize that Theorem 3 is a simple corollary of Proposition 1 and of the WIs Eqs.(1.32)-(11.33). In fact, from Eq.(1.33) computed at $\mathbf{p}=\left(0, p_{1}, 0\right)$, we find

$$
\hat{K}_{1 i}\left(0, p_{1}, 0\right)+\lim _{\beta, L \rightarrow \infty} \frac{1}{L^{2}}\left\langle\left[\hat{\Delta}_{\left(p_{1}, 0\right),\left(-p_{1}, 0\right)}\right]_{1 i}\right\rangle_{\beta, L}=0
$$

that implies, using the continuity of $\hat{K}_{l m}(\mathbf{p})$ at $\mathbf{p}=\mathbf{0}$ stated in Proposition 1 ,

$$
\lim _{\mathbf{p} \rightarrow \mathbf{0}} \hat{K}_{1 i}(\mathbf{p})=-\Delta_{1 i}
$$

and a similar argument shows that

$$
\lim _{\mathbf{p} \rightarrow \mathbf{0}} \hat{K}_{l m}(\mathbf{p})=-\Delta_{l m}
$$

for all $l, m \in\{1,2\}$. Therefore, using again the continuity at $\mathbf{p}=\mathbf{0}$ of the current-current function and the definition of conductivity, we can rewrite

$$
\sigma_{l m}=-\frac{2}{3 \sqrt{3}} \lim _{p_{0} \rightarrow 0^{+}} \frac{1}{p_{0}}\left[\hat{K}_{l m}\left(p_{0}, \overrightarrow{0}\right)-\hat{K}_{l m}(\mathbf{0})\right] .
$$

We now use the decomposition Eq.(11.48) to rewrite the latter equation as

$$
\begin{aligned}
\sigma_{l m}= & -\frac{2}{3 \sqrt{3}} \lim _{p_{0} \rightarrow 0^{+}} \frac{1}{p_{0}} \frac{Z_{l} Z_{m}}{Z^{2}}\left[\left\langle\hat{\jmath}_{\left(p_{0}, \overrightarrow{0}\right), l} ; \hat{\jmath}_{-\left(p_{0}, \overrightarrow{0}\right), m}\right\rangle^{0, v_{F}}-\left\langle\hat{\jmath}_{\mathbf{0}, l} ; \hat{\jmath}_{\mathbf{0}, m}\right\rangle^{0, v_{F}}\right] \\
& -\frac{2}{3 \sqrt{3}} \lim _{p_{0} \rightarrow 0^{+}} \frac{1}{p_{0}}\left[R_{l m}\left(p_{0}, \overrightarrow{0}\right)-R_{l m}(\mathbf{0})\right] .
\end{aligned}
$$


Now, using the identity $Z_{i}=Z v_{F}$, see Eq.(1145), we conclude that the limit in the first line reduces to the computation of the free conductivity Eq.(11.38) with $v_{0}$ replaced by $v_{F}$ : however, since the result does not depend on the Fermi velocity, from the first line we simply get $\left(e^{2} / h\right)(\pi / 2) \delta_{l m}$. On the other hand, the limit in the second line, using the continuous differentiability of $R(\mathbf{p})$, reduces to $-\frac{2}{3 \sqrt{3}} \partial_{0} R(\mathbf{0})$, which is zero, simply because $R(\mathbf{p})$ is even in $\mathbf{p}$.

This concludes the proof of Theorem 3, once that Proposition 1 is given. The rest of the paper will be devoted to proofs of Theorem 2 and Proposition 1.

\section{REGULARITY OF THE CURRENT-CURRENT CORRELATIONS}

In this section we prove Theorem 2 and Proposition 1. We will use an extension of the method discussed in [11]. We will assume the reader familiar with the proof in [11] and we will only describe in detail the new aspects of the construction, as compared to the one in [11]. Still, we will try to be as self-consistent as possible, possibly giving reference to a well-defined section of [11] for the few technical aspects that will not be fully reproduced here.

\section{A. Grassman Integral representation for the correlation functions}

The goal is to prove analyticity in $U$ and regularity in $\mathbf{k}, \mathbf{p}$ of the three-point and currentcurrent functions. We remind the reader that the proof of analyticity and the control of regularity of the Schwinger functions (in particular, of the two-points function) has already appeared in [11], see in particular Section 3.4 of [11]. The starting point of our construction is a representation of the generating function for correlations in terms of a Grassmann functional integral, completely analogous to the one used in [11] to write the generating function for the Schwinger functions. The Grassmann functional integral we are intersted in is defined as follows.

Let $M \in \mathbb{N}$ and $\chi_{0}(t)$ a smooth compact support function that is 1 for $t \leq a_{0}$ and 0 for $t \geq 2 a_{0}$, with $a_{0}$ a constant that can be chosen equal to, e.g., $1 / 3$ (see the condition on $a_{0}$ appearing after Eq.(3.41) of [11] and read it for $\gamma=2$ ). Let $\mathcal{B}_{\beta, L}^{*}=$ $\left\{2 \pi \beta^{-1}\left(\mathbb{Z}+\frac{1}{2}\right) \cap\left\{k_{0}: \chi_{0}\left(2^{-M}\left|k_{0}\right|\right)>0\right\}\right\} \times \mathcal{B}_{L}$. We consider the finite Grassmann algebra 
generated by the Grassmannian variables $\left\{\hat{\Psi}_{\mathbf{k}, \sigma, \rho}^{ \pm}\right\}_{\mathbf{k} \in \mathcal{B}_{\beta, L}^{*}}^{\sigma=\uparrow \downarrow, \rho=1,2}$ and a Grassmann integration $\int\left[\prod_{\mathbf{k} \in \mathcal{B}_{\beta, L}^{*}} \prod_{\sigma=\uparrow \downarrow}^{\rho=1,2} d \hat{\Psi}_{\mathbf{k}, \sigma, \rho}^{+} d \hat{\Psi}_{\mathbf{k}, \sigma, \rho}^{-}\right]$defined as the linear operator on the Grassmann algebra such that, given a monomial $Q\left(\hat{\Psi}^{-}, \hat{\Psi}^{+}\right)$in the variables $\hat{\Psi}_{\mathbf{k}, \sigma, \rho}^{ \pm}$, its action on $Q\left(\hat{\Psi}^{-}, \hat{\Psi}^{+}\right)$is 0 except in the case $Q\left(\hat{\Psi}^{-}, \hat{\Psi}^{+}\right)=\prod_{\mathbf{k} \in \mathcal{B}_{\beta, L}^{*}} \prod_{\sigma=\uparrow \downarrow}^{\rho=1,2} \hat{\Psi}_{\mathbf{k}, \sigma, \rho}^{-} \hat{\Psi}_{\mathbf{k}, \sigma, \rho}^{+}$, up to a permutation of the variables. In this case the value of the integral is determined, by using the anticommuting properties of the variables, by the condition

$$
\int\left[\prod_{\mathbf{k} \in \mathcal{B}_{\beta, L}^{*}} \prod_{\sigma=\uparrow \downarrow}^{\rho=1,2} d \hat{\Psi}_{\mathbf{k}, \sigma, \rho}^{+} d \hat{\Psi}_{\mathbf{k}, \sigma, \rho}^{-}\right] \prod_{\mathbf{k} \in \mathcal{B}_{\beta, L}^{*}} \prod_{\sigma=\uparrow \downarrow}^{\rho=1,2} \hat{\Psi}_{\mathbf{k}, \sigma, \rho}^{-} \hat{\Psi}_{\mathbf{k}, \sigma, \rho}^{+}=1
$$

Let us define the free propagator matrix $\hat{g}_{\mathbf{k}}$ as

$$
\hat{g}_{\mathbf{k}}=\chi_{0}\left(2^{-M}\left|k_{0}\right|\right)\left(\begin{array}{cc}
-i k_{0} & -v_{0} \Omega^{*}(\vec{k}) \\
-v_{0} \Omega(\vec{k}) & -i k_{0}
\end{array}\right)^{-1}
$$

and the "Gaussian integration" $P(d \psi)$ as

$$
\begin{gathered}
P(d \Psi)=\left[\prod_{\mathbf{k} \in \mathcal{B}_{\beta, L}^{*}}^{\sigma=\uparrow \downarrow} \frac{-\beta^{2} L^{4}\left[\chi_{0}\left(\gamma^{-M}\left|k_{0}\right|\right)\right]^{2}}{k_{0}^{2}+v_{0}^{2}|\Omega(\vec{k})|^{2}} d \hat{\Psi}_{\mathbf{k}, \sigma, 1}^{+} d \hat{\Psi}_{\mathbf{k}, \sigma, 1}^{-} d \hat{\Psi}_{\mathbf{k}, \sigma, 2}^{+} d \hat{\Psi}_{\mathbf{k}, \sigma, 2}^{-}\right] \\
\cdot \exp \left\{-\left(\beta L^{2}\right)^{-1} \sum_{\mathbf{k} \in \mathcal{B}_{\beta, L}^{*}}^{\sigma=\uparrow \downarrow} \hat{\Psi}_{\mathbf{k}, \sigma}^{+} \hat{g}_{\mathbf{k}}^{-1} \hat{\Psi}_{\mathbf{k}, \sigma}^{-}\right\}
\end{gathered}
$$

Let us also introduce the generating function

$$
\mathcal{W}_{M, \beta, L}(A, \phi)=\log \int P(d \Psi) e^{\mathcal{V}(\Psi)+(\Psi, \phi)+(A, J)}
$$

where

$$
\begin{aligned}
& \mathcal{V}(\Psi)=-U \sum_{\rho=1,2} \int_{(\beta, L)} d \mathbf{x} \Psi_{\mathbf{x}, \uparrow, \rho}^{+} \Psi_{\mathbf{x}, \uparrow, \rho}^{-} \Psi_{\mathbf{x}, \downarrow, \rho}^{+} \Psi_{\mathbf{x}, \downarrow, \rho}^{-}, \\
& (\Psi, \phi)=\sum_{\sigma=\uparrow \downarrow} \int_{(\beta, L)} d \mathbf{x}\left(\Psi_{\mathbf{x}, \sigma}^{+} \phi_{\mathbf{x}, \sigma}^{-}+\phi_{\mathbf{x}, \sigma}^{+} \Psi_{\mathbf{x}, \sigma}^{-}\right), \\
& (A, J)=\sum_{\tau= \pm} \int_{(\beta, L)} d \mathbf{x} A_{\mathbf{x}, \tau} J_{\mathbf{x}}^{\tau}+v_{0} \sum_{j=1,2,3} \int_{(\beta, L)} d \mathbf{x} A_{\mathbf{x}, j} J_{\mathbf{x}}^{j},
\end{aligned}
$$

and, in the last line, denoting by $\sigma_{1}, \sigma_{2}, \sigma_{3}$ the standard Pauli matrices,

$$
\sigma_{1}=\left(\begin{array}{cc}
0 & 1 \\
1 & 0
\end{array}\right), \quad \sigma_{2}=\left(\begin{array}{cc}
0 & -i \\
i & 0
\end{array}\right), \quad \sigma_{3}=\left(\begin{array}{cc}
1 & 0 \\
0 & -1
\end{array}\right)
$$


and defining $n_{ \pm}=\left(1 \pm \sigma_{3}\right) / 2$ and $\sigma_{ \pm}=\left(\sigma_{1} \pm i \sigma_{2}\right) / 2$,

$$
J_{\mathbf{x}}^{ \pm}=\sum_{\sigma=\uparrow \downarrow} \Psi_{\mathbf{x}, \sigma}^{+} n_{ \pm} \Psi_{\mathbf{x}, \sigma}^{-}, \quad J_{\mathbf{x}}^{j}=\frac{2 i}{3} \sum_{\sigma=\uparrow \downarrow}\left[\Psi_{\mathbf{x}, \sigma}^{+} \sigma_{+} \Psi_{\mathbf{x}+\boldsymbol{\delta}_{j}, \sigma}^{-}-\Psi_{\mathbf{x}+\boldsymbol{\delta}_{j}, \sigma}^{+} \sigma_{-} \Psi_{\mathbf{x}, \sigma}^{+}\right] .
$$

We will be particularly concerned with the three-points functions:

$$
\bar{G}_{2,1 ; \sharp,}^{M, \beta}(\mathbf{z} ; \mathbf{x}, \mathbf{y})=\left.e \frac{\partial}{\partial A_{\mathbf{z}, \sharp}} \frac{\partial^{2}}{\partial \phi_{\mathbf{y}, \sigma}^{-} \partial \phi_{\mathbf{x}, \sigma}^{+}} \mathcal{W}_{M, \beta, L}(A, \phi)\right|_{A=\phi=0}, \quad \sharp \in\{+,-, 1,2,3\},
$$

and with the current-current response functions:

$$
\bar{K}_{\sharp, b}^{M, \beta, L}(\mathbf{x} ; \mathbf{y})=\left.e^{2} \frac{\partial^{2}}{\partial A_{\mathbf{x}, \sharp} \partial A_{\mathbf{y}, b}} \mathcal{W}_{M, \beta, L}(A, \phi)\right|_{A=\phi=0}, \quad \sharp, b \in\{+,-, 1,2,3\} .
$$

The connection between these functions and the corresponding objects evaluated in he Hamiltonian model of Section $1 \mathrm{~A}$ is provided by the following proposition, which is the analogue of Proposition 1 of [11].

Proposition 2. For any $\beta, L<+\infty$, assume that there exists $U_{0}$ independent of $\beta$ and $L$ such that the three-points functions $G_{2,1 ; \#}^{M, \beta, L}(\mathbf{z} ; \mathbf{x}, \mathbf{y})$ and current-current response functions $K_{\sharp, b}^{M, \beta, L}(\mathbf{x} ; \mathbf{y})$ at distinct space-time points are analytic in the complex domain $|U| \leq U_{0}$, uniformly convergent as $M \rightarrow \infty$. Then, if $|U| \leq U_{0}$ and $\mathbf{z}, \mathbf{x}, \mathbf{y}$ are three distinct spacetime points,

$$
\begin{aligned}
e\left\langle\rho_{\mathbf{z}}^{\tau} ; \psi_{\mathbf{x}, \sigma}^{-} \psi_{\mathbf{y}, \sigma}^{+}\right\rangle_{\beta, L} & =\lim _{M \rightarrow \infty} \bar{G}_{2,1 ; \tau}^{M, \beta, L}(\mathbf{z} ; \mathbf{x}, \mathbf{y}), & & \tau= \pm \\
e v_{0}\left\langle J_{\mathbf{z}}^{j} ; \psi_{\mathbf{x}, \sigma}^{-} \psi_{\mathbf{y}, \sigma}^{+}\right\rangle_{\beta, L} & =\lim _{M \rightarrow \infty} \bar{G}_{2,1 ; j}^{M, \beta, L}(\mathbf{z} ; \mathbf{x}, \mathbf{y}), & & j=1,2,3,
\end{aligned}
$$

where: the averages in the l.h.s are defined as in Eq.(11.16) and following lines; $\rho_{\mathbf{x}}^{A}=$ $\sum_{\sigma} a_{\mathbf{x}, \sigma}^{+} a_{\mathbf{x}, \sigma}^{-}$and $\rho_{\mathbf{x}}^{B}=\sum_{\sigma} b_{\mathbf{x}+\delta_{1}, \sigma}^{+} b_{\mathbf{x}+\delta_{1}, \sigma}^{-}$. Moreover, if $\mathbf{x}, \mathbf{y}$ are two distinct space-time points,

$$
\begin{aligned}
e^{2}\left\langle\rho_{\mathbf{x}}^{\tau} ; \rho_{\mathbf{y}}^{\tau^{\prime}}\right\rangle_{\beta, L} & =\lim _{M \rightarrow \infty} \bar{K}_{\tau, \tau^{\prime}}^{M, \beta, L}(\mathbf{x} ; \mathbf{y}), & & \tau, \tau^{\prime}= \pm, \\
e^{2} v_{0}\left\langle\rho_{\mathbf{x}}^{\tau} ; J_{\mathbf{y}}^{j}\right\rangle_{\beta, L} & =\lim _{M \rightarrow \infty} \bar{K}_{\tau, \beta}^{M, \beta}(\mathbf{x} ; \mathbf{y}), & & \tau= \pm, \quad j=1,2,3, \\
e^{2} v_{0}^{2}\left\langle J_{\mathbf{x}}^{j} ; J_{\mathbf{y}}^{j^{\prime}}\right\rangle_{\beta, L} & =\lim _{M \rightarrow \infty} \bar{K}_{j, j^{\prime}}^{M, \beta, L}(\mathbf{x} ; \mathbf{y}), & & j, j^{\prime}=1,2,3,
\end{aligned}
$$

The proof of this statement is completely analogous to the one in Appendix B of [11] and will not be repeated here. Note that, once that the various correlation functions in Proposition 2 are known, we can reconstruct the functions $\hat{K}_{\mu \nu}^{\beta, L}(\mathbf{p})$ and $\hat{G}_{2,1 ; \mu}^{\beta, L}(\mathbf{k}, \mathbf{p})$ in Eqs.(11.19)-(11.21) 
simply by Fourier transformation (provided that the correlation functions in Proposition 2 have good enough decay properties so that the Fourier transform is well defined), e.g.,

$$
\begin{aligned}
& \hat{G}_{2,1 ; 0}^{\beta, L}(\mathbf{k}, \mathbf{p})=\frac{e}{\beta L^{2}} \int_{(\beta, L)} d \mathbf{x} \int_{(\beta, L)} d \mathbf{y} \int_{(\beta, L)} d \mathbf{z} e^{i \mathbf{k}(\mathbf{x}-\mathbf{y})+i \mathbf{p}(\mathbf{x}-\mathbf{z})}\left\langle\left(\rho_{\mathbf{z}}^{A}+e^{-i \vec{p} \vec{\delta}_{1}} \rho_{\mathbf{z}+\boldsymbol{\delta}_{1}}^{B}\right) ; \psi_{\mathbf{x}, \sigma}^{-} \psi_{\mathbf{y}, \sigma}^{+}\right\rangle_{\beta, L}, \\
& \hat{G}_{2,1 ; l}^{\beta, L}(\mathbf{k}, \mathbf{p})=\frac{e v_{0}}{\beta L^{2}} \int_{(\beta, L)} d \mathbf{x} \int_{(\beta, L)} d \mathbf{y} \int_{(\beta, L)} d \mathbf{z} e^{i \mathbf{k}(\mathbf{x}-\mathbf{y})+i \mathbf{p}(\mathbf{x}-\mathbf{z})} \sum_{j=1}^{3}\left\langle\left(\vec{\delta}_{j}\right)_{l} \eta_{\vec{p}}^{j} J_{\mathbf{z}}^{j} ; \psi_{\mathbf{x}, \sigma}^{-} \psi_{\mathbf{y}, \sigma}^{+}\right\rangle_{\beta, L} ;(2,17)
\end{aligned}
$$

similar formulas are valid for $\hat{K}_{\mu \nu}^{\beta, L}(\mathbf{p})$.

\section{B. Renormalization Group}

The naive perturbative expansion in $U$ of $\mathcal{W}_{M, \beta, L}(A, \phi)$ is affected by infrared divergences due to the singularity at the Fermi points of the free propagator $S_{0}(\mathbf{k})$, see Eq.(11.36). The case with $A=0$ has been studied in detail in [11], where it has been shown that the apparent divergences affecting the naive perturbation series can be cured by proper iterative resummations; these allowed us to recast the original expansion into a new convergent expansion (uniformly in $M, \beta, L$ ) involving a sequence of effective parameters $Z_{h}, v_{h}$, playing the role of effective wave function renormalization and Fermi velocity at momentum scale $2^{h}, h \leq 0$, relative to the Fermi points. The iterative resummations can be implemented by using constructive fermionic Renormalization Group ( $R G$ ) techniques; a key point of the analysis, which make the construction of the ground state possible, is the fact that densitydensity interactions are irrelevant in a RG sense. In this section we review the iterative integration scheme used to compute $\mathcal{W}_{M, \beta, L}(A, \phi)$, with particular emphasis to the novelties due to presence of the external field $A$.

\section{The ultraviolet integration.}

Proceeding as in Sections 3.2 and 3.4 of [11], we decompose the propagators $\hat{g}(\mathbf{k})$ into sums of two propagators supported in the regions of $k_{0}$ "large" and "small". The regions of $k_{0}$ large and small are defined in terms of the smooth support function $\chi(t)$; the constant $a_{0}$ entering in its definition is chosen so that the supports of $\chi_{0}\left(\sqrt{k_{0}^{2}+\left|\vec{k}-\vec{p}_{F}^{+}\right|}\right)$and $\chi_{0}\left(\sqrt{k_{0}^{2}+\left|\vec{k}-\overrightarrow{p_{F}}\right|}\right)$ are disjoint (e.g., $a_{0}=1 / 3$ is fine). We rewrite $\hat{g}(\mathbf{k})$ as

$$
\hat{g}(\mathbf{k})=\hat{g}^{(u . v .)}(\mathbf{k})+\hat{g}^{(i . r .)}(\mathbf{k})
$$


where, setting $\mathbf{p}_{F}^{\omega}=\left(0, \vec{p}_{F}^{\omega}\right)$ with $\omega= \pm$ :

$$
\hat{g}^{(u . v .)}(\mathbf{k})=\hat{g}(\mathbf{k})-\hat{g}^{(i . r .)}(\mathbf{k}), \quad g^{(i . r .)}(\mathbf{k})=\sum_{\omega= \pm} \chi_{0}\left(\left|\mathbf{k}-\mathbf{p}_{F}^{\omega}\right|\right) \hat{g}(\mathbf{k}),
$$

Defining $V(A, \Psi, \phi)=\mathcal{V}(\Psi)+(\Psi, \phi)+(A, J)$, we can rewrite

$$
e^{\mathcal{W}_{M, \beta, L}(A, \phi)}=\int P(d \Psi) e^{V(A, \Psi, \phi)}=\int P\left(d \Psi^{(i . r .)}\right) \int P\left(d \Psi^{(u . v .)}\right) \mathrm{e}^{V\left(A, \Psi^{(i . r .)}+\Psi^{(u . v .)}, \phi\right)},
$$

where $P\left(d \Psi^{(i . r .)}\right)$ and $P\left(d \Psi^{(u . v .)}\right)$ are the Gaussian integrations associated to the propagators $\hat{g}^{(i . r .)}(\mathbf{k})$ and $\hat{g}^{(u . v .)}(\mathbf{k})$, respectively. Using Eq.(2[20) we can further rewrite the generating functional as:

$$
\begin{aligned}
e^{\mathcal{W}_{M, \beta, L}(A, \phi)} & =\int P\left(d \Psi^{(i . r .)}\right) \exp \left\{\sum_{n \geq 1} \frac{1}{n !} \mathcal{E}_{u . v .}^{T}\left(V\left(A, \Psi^{(i . r .)}+\cdot, \phi\right) ; n\right)\right\}= \\
& =: e^{-\beta L^{2} F_{0, M}} \int P\left(d \Psi^{(i . r .)}\right) e^{\mathcal{V}_{M}\left(\Psi^{(i . r .)}\right)+B_{M}\left(A, \Psi^{(i . r .)}, \phi\right)+\left(\phi, \Psi^{(i . r .)}\right)+\left(A, J^{(i . r .)}\right)}
\end{aligned}
$$

where: $\mathcal{E}_{u . v .}^{T}$ is the truncated expectation with respect to the propagator $\hat{g}^{(u . v .)}(\mathbf{k}) ; F_{0, M}$ is a constant; $\mathcal{V}_{M}$ is the effective potential on scale $0 ; B_{M}\left(A, \Psi^{(i . r .)}, \phi\right)$ collects the terms depending on $A, \phi$ generated by the ultraviolet integration; $J^{(i . r .)}$ is defined in the same way as $J$ (see Eq.(2.9) ) with $\Psi$ replaced by $\Psi^{(i . r .)}$. As proved in [1]] (see Eq.(3.36) and Lemma 2 of [11]), the effective potential $\mathcal{V}_{M}$ can be written as

$$
\begin{gathered}
\mathcal{V}_{M}\left(\Psi^{(i . r .)}\right)=\sum_{n=1}^{\infty}\left(\beta L^{2}\right)^{-2 n} \sum_{\sigma_{1}, \ldots, \sigma_{n}=\uparrow \downarrow} \sum_{\rho_{1}, \ldots, \rho_{2 n}=1,2} \sum_{\mathbf{k}_{1}, \ldots, \mathbf{k}_{2 n}}\left[\prod_{j=1}^{n} \hat{\Psi}_{\mathbf{k}_{2 j-1}, \sigma_{j}, \rho_{2 j-1}}^{(i . r .)+} \hat{\Psi}_{\mathbf{k}_{2 j}, \sigma_{j}, \rho_{2 j}}^{(i . r .)-}\right] . \\
\cdot \hat{W}_{M ; 2 n ; \underline{\rho}}\left(\mathbf{k}_{1}, \ldots, \mathbf{k}_{2 n-1}\right) \delta\left(\sum_{j=1}^{n}\left(\mathbf{k}_{2 j-1}-\mathbf{k}_{2 j}\right)\right)
\end{gathered}
$$

where $\underline{\rho}=\left(\rho_{1}, \ldots, \rho_{2 n}\right)$ and we used the notation

$$
\delta(\mathbf{k})=\delta(\vec{k}) \delta\left(k_{0}\right), \quad \delta(\vec{k})=L^{2} \sum_{n_{1}, n_{2} \in \mathbb{Z}} \delta_{\vec{k}, n_{1} \vec{G}_{1}+n_{2} \vec{G}_{2}}, \quad \delta\left(k_{0}\right)=\beta \delta_{k_{0}, 0},
$$

with $\vec{G}_{1}, \vec{G}_{2}$ the basis of $\Lambda^{*}$ defined after Eq.(11.3). Moreover (see Lemma 2 of [11]), the constant $F_{0, M}$ and the kernels $\hat{W}_{M ; 2 n ; \underline{\rho}}$ are given by power series in $U$, convergent in the complex disc $|U| \leq U_{0}$, for $U_{0}$ small enough and independent of $\beta, L, M$; after Fourier transform, the $\mathbf{x}$-space counterparts of the kernels $\hat{W}_{M ; 2 n ; \underline{\rho}}$ satisfy the following bounds:

$$
\int d \mathbf{x}_{1} \cdots d \mathbf{x}_{2 n}\left[\prod_{1 \leq i<j \leq 2 n}|| \mathbf{x}_{i}-\left.\mathbf{x}_{j}\right|^{m_{i, j}}\right]\left|W_{M ; 2 n ; \underline{\rho}}\left(\mathbf{x}_{1}, \ldots, \mathbf{x}_{2 n}\right)\right| \leq \beta|\Lambda| C_{m}^{n}|U|^{\max \{1, n-1\}},
$$


for some constant $C_{m}>0$, where $m=\sum_{1 \leq i<j \leq 2 n} m_{i, j}$. The limits $F_{0}=\lim _{M \rightarrow \infty} F_{0, M}$ and $W_{2 n ; \underline{\rho}}\left(\mathbf{x}_{1}, \ldots, \mathbf{x}_{2 n}\right)=\lim _{M \rightarrow \infty} W_{M ; 2 n ; \underline{\rho}}\left(\mathbf{x}_{1}, \ldots, \mathbf{x}_{2 n}\right)$ exist and are reached uniformly in $M$, so that, in particular, the limiting functions are analytic in the same domain $|U| \leq U_{0}$.

The functional $B_{M}\left(A, \Psi^{(i . r .)}, \phi\right)$ admits very similar representation and bounds, i.e.,

$$
\begin{aligned}
& B_{M}\left(A, \Psi^{(i . r .)}, \phi\right)=\sum_{n, m=0}^{\infty} \frac{1}{\left(\beta L^{2}\right)^{2 n+m}} \sum_{\underline{\underline{\sigma}, \underline{\rho}} \underline{\underline{\mathbf{k}}, \underline{\underline{p}}}} \sum_{\underline{\underline{\gamma}} \underline{\underline{p}}}\left[\prod_{j=1}^{n} \hat{\varphi}_{\mathbf{k}_{2 j-1}, \sigma_{j}, \rho_{2 j-1}, \gamma_{2 j-1}}^{+} \hat{\varphi}_{\mathbf{k}_{2 j}, \sigma_{j}, \rho_{2 j}, \gamma_{2 j}}^{-}\right]\left[\prod_{i=1}^{m} \hat{A}_{\sharp i, \mathbf{p}_{i}}\right] \\
& \cdot \hat{W}_{M ; 2 n, m ; \underline{\rho, \sharp} ; \underline{\gamma}}\left(\left\{\mathbf{k}_{j}\right\},\left\{\mathbf{p}_{i}\right\}\right) \delta\left(\sum_{j=1}^{n}\left(\mathbf{k}_{2 j-1}-\mathbf{k}_{2 j}\right)-\sum_{i=1}^{m} \mathbf{p}_{i}\right) \text {, }
\end{aligned}
$$

where $\underline{\sigma}:=\left(\sigma_{1}, \ldots, \sigma_{n}\right), \underline{\sharp}:=\left(\sharp_{1}, \ldots, \sharp_{m}\right)$ (with $\left.\sharp_{i} \in\{+,-, 1,2,3\}\right), \underline{\gamma}=\left(\gamma_{1}, \ldots, \gamma_{2 n}\right)$ (with $\gamma_{i} \in\{$ ext,int $\left.\}\right), \underline{\mathbf{k}}=\left(\mathbf{k}_{1}, \ldots, \mathbf{k}_{2 n}\right), \underline{\mathbf{p}}=\left(\mathbf{p}_{1}, \ldots, \mathbf{p}_{m}\right)$ and the field $\hat{\varphi}$ can be either $\Psi^{(i . r .)}$ or $\phi$, depending on the label $\gamma$, i.e., $\hat{\varphi}_{\mathbf{k}, \sigma, e x t}^{ \pm}=\hat{\phi}_{\mathbf{k}, \sigma}^{ \pm}$and $\hat{\varphi}_{\mathbf{k}, \sigma, i n t}^{ \pm}=\hat{\Psi}_{\mathbf{k}, \sigma}^{(i . r .) \pm}$. The kernels $\hat{W}_{M ; 2 n, m ; \underline{\rho}, \sharp ; \underline{\gamma}}$ are analytic in $U$, they admit bounds analogous to Eq.(2.24), uniformly in $\underline{\gamma}$ and $M$, and converge uniformly as $M \rightarrow \infty$ to limiting kernels denoted by $\hat{W}_{2 n, m ; \underline{\rho}, \sharp ; \underline{\gamma}}$ (for this reason, the label $M$ will not play any important role in the following and will be dropped from now on). The proof of these claim goes along the same lines as the proof of Lemma 2 in [11] and will not repeated here.

The kernels $\hat{W}_{2 n, m ; \underline{\rho}, \sharp ; \underline{\gamma}}$ satisfy a number of symmetry properties inherited from the symmetries of the action and of the Gaussian integration, described and proved in Appendix B. In particular, thinking of $\hat{W}_{2 n, m ; \sharp ; \underline{\gamma}}$ as tensors with entries $\hat{W}_{2 n, m ; \underline{\underline{\beta}, \sharp ; \underline{\gamma}}}$ and defin$\operatorname{ing} \hat{W}_{2, \omega}\left(\mathbf{k}^{\prime}\right):=W_{2,0 ;(i n t, i n t)}\left(\mathbf{k}^{\prime}+\mathbf{p}_{F}^{\omega}, \mathbf{k}^{\prime}+\mathbf{p}_{F}^{\omega}\right), \hat{W}_{\sharp, b}(\mathbf{p}):=\hat{W}_{0,2 ; \sharp, b}(\mathbf{p},-\mathbf{p}), \hat{W}_{\sharp, \omega}\left(\mathbf{k}^{\prime}, \mathbf{p}\right):=$ $\hat{W}_{2,1 ; \sharp ; \text { int }, i n t)}\left(\mathbf{k}^{\prime}+\mathbf{p}_{F}^{\omega}+\mathbf{p}, \mathbf{k}^{\prime}+\mathbf{p}_{F}^{\omega}, \mathbf{p}\right)$,

$$
\begin{aligned}
\hat{W}_{2}\left(\mathbf{k}^{\prime}\right) & =-i z_{0} k_{0}^{\prime}+z_{1}\left(k_{1}^{\prime} \sigma_{2}+\omega k_{2}^{\prime} \sigma_{1}\right)+O\left(\left|\mathbf{k}^{\prime}\right|^{2}\right), \\
\hat{W}_{\tau, \tau^{\prime}}(\mathbf{p}) & =e^{-i \frac{\vec{p}}{2} \vec{\delta}_{1}\left(\tau-\tau^{\prime}\right)}\left(a+a^{\prime} \tau \tau^{\prime}\right)+O\left(|\mathbf{p}|^{2}\right), \\
\hat{W}_{\tau, j}(\mathbf{p}) & =b \tau p_{0}+O\left(|\mathbf{p}|^{2}\right), \\
\hat{W}_{j, j^{\prime}}(\mathbf{p}) & =e^{i \frac{\vec{p}}{2}\left(\vec{\delta}_{j}-\vec{\delta}_{j^{\prime}}\right)}\left(c \delta_{j, j^{\prime}}+c^{\prime}\right)+O\left(|\mathbf{p}|^{2}\right), \\
\hat{W}_{\tau, \omega}\left(\mathbf{k}^{\prime}, \mathbf{p}\right) & =\lambda_{0}+\lambda_{3} \tau \sigma_{3}+O\left(\left|\mathbf{k}^{\prime}\right|+|\mathbf{p}|\right), \\
\hat{W}_{j, \omega}\left(\mathbf{k}^{\prime}, \mathbf{p}\right) & =\lambda_{1} e^{i \omega \frac{2 \pi}{3}(j-1) \sigma_{3}} \sigma_{2}+O\left(\left|\mathbf{k}^{\prime}\right|+|\mathbf{p}|\right),
\end{aligned}
$$

where the constants $z_{0}, z_{1}, a, a^{\prime}, b, c, c^{\prime}, \lambda_{0}, \lambda_{1}, \lambda_{3}$ are all real. The proof of Eq.(2.26) is in Lemma 3 of [11], while the proof of the remaining relations is in Appendix C. 


\section{The infrared integration.}

After the integration of the ultraviolet modes, we decompose the infrared propagator as a sum of two quasi-particle propagators:

$$
g^{(i . r .)}(\mathbf{x}-\mathbf{y})=\sum_{\omega= \pm} e^{-i \mathbf{p}_{F}^{\omega}(\mathbf{x}-\mathbf{y})} g_{\omega}^{(\leq 0)}(\mathbf{x}-\mathbf{y})
$$

where, if $\mathbf{k}^{\prime}=\left(k_{0}, \vec{k}^{\prime}\right)$,

$$
g_{\omega}^{(\leq 0)}(\mathbf{x}-\mathbf{y})=\frac{1}{\beta L^{2}} \sum_{\mathbf{k}^{\prime} \in \mathcal{B}_{\beta, L}^{\omega}} \chi_{0}\left(\left|\mathbf{k}^{\prime}\right|\right) e^{-i \mathbf{k}^{\prime}(\mathbf{x}-\mathbf{y})}\left(\begin{array}{cc}
-i k_{0} & -v_{0} \Omega^{*}\left(\vec{k}^{\prime}+\vec{p}_{F}^{\omega}\right) \\
-v_{0} \Omega\left(\vec{k}^{\prime}+\vec{p}_{F}^{\omega}\right) & -i k_{0}
\end{array}\right)^{-1}
$$

and $\mathcal{B}_{\beta, L}^{\omega}=\left\{2 \pi \beta^{-1}\left(\mathbb{Z}+\frac{1}{2}\right) \cap\left\{k_{0}: \chi_{0}\left(2^{-M}\left|k_{0}\right|\right)>0\right\}\right\} \times\left\{\frac{n_{1}}{L} \vec{G}_{1}+\frac{n_{2}}{L} \vec{G}_{2}-\vec{p}_{F}^{\omega}, 0 \leq n_{1}, n_{2}<L\right\}$. Correspondingly, we rewrite $\Psi^{(i . r .)}$ and $\phi^{ \pm}$as sums of two independent Grassmann fields, $\Psi_{\mathbf{x}, \sigma}^{(i . r .) \pm}=\sum_{\omega= \pm} e^{ \pm i \mathbf{p}_{F}^{\omega} \mathbf{x}} \Psi_{\mathbf{x}, \sigma, \omega}^{(\leq 0) \pm}$ and $\phi_{\mathbf{x}, \sigma}^{ \pm}=\sum_{\omega= \pm} e^{ \pm i \mathbf{p}_{F}^{\omega} \mathbf{x}} \phi_{\mathbf{x}, \sigma, \omega}^{ \pm}$, and rewrite the generating functional as (dropping systematically the $M$ label):

$$
\begin{aligned}
& e^{\mathcal{W}_{\beta, L}(A, \phi)}= \\
& \quad=e^{-\beta L^{2} F_{0}+S^{(\geq 0)}(A, \phi)} \int P_{\chi_{0}, C_{0}}\left(d \Psi^{(\leq 0)}\right) e^{\mathcal{V}^{(0)}\left(\Psi^{(\leq 0)}\right)+B^{(0)}\left(A, \Psi^{(\leq 0)}, \phi\right)+\left(A \Psi^{(\leq 0)}, T_{1} \Psi^{(\leq 0)}\right)+\left(\phi, \Psi^{(\leq 0)}\right)},
\end{aligned}
$$

where:

$$
\begin{aligned}
P_{\chi_{0}, C_{0}}\left(d \Psi^{(\leq 0)}\right) & =\mathcal{N}_{0}{ }^{-1}\left[\prod_{\mathbf{k}^{\prime} \in \mathcal{B}_{\beta, L}^{\omega}}^{\chi_{0}\left(\left|\mathbf{k}^{\prime}\right|\right)>0} \prod_{\sigma, \omega, \rho} d \hat{\Psi}_{\mathbf{k}^{\prime}, \sigma, \rho, \omega}^{(\leq 0)+} d \hat{\Psi}_{\mathbf{k}^{\prime}, \sigma, \rho, \omega}^{(\leq 0)-}\right] \\
& \cdot \exp \left\{-\left(\beta L^{2}\right)^{-1} \sum_{\omega= \pm}^{\sigma=\uparrow \downarrow} \sum_{\mathbf{k}^{\prime} \in \mathcal{B}_{\beta, L}^{\omega}}^{\left(\left|\mathbf{k}^{\prime}\right|\right)>0} \chi_{0}^{-1}\left(\left|\mathbf{k}^{\prime}\right|\right) \hat{\Psi}_{\mathbf{k}^{\prime}, \sigma, \omega}^{(\leq 0)+} C_{0, \omega}\left(\mathbf{k}^{\prime}\right) \hat{\Psi}_{\mathbf{k}^{\prime}, \sigma, \omega}^{(\leq 0)-}\right\}
\end{aligned}
$$

and

$$
\begin{aligned}
C_{0, \omega}\left(\mathbf{k}^{\prime}\right) & =\left(\begin{array}{cc}
-i k_{0} & -v_{0} \Omega^{*}\left(\vec{k}^{\prime}+\vec{p}_{F}^{\omega}\right) \\
-v_{0} \Omega\left(\vec{k}^{\prime}+\vec{p}_{F}^{\omega}\right) & -i k_{0}
\end{array}\right)= \\
& =: Z_{0}\left(\begin{array}{cc}
-i k_{0} & v_{0}\left(i k_{1}^{\prime}-\omega k_{2}^{\prime}\right) \\
v_{0}\left(-i k_{1}^{\prime}-\omega k_{2}^{\prime}\right) & -i k_{0}
\end{array}\right)\left(1+R_{0, \omega}\left(\mathbf{k}^{\prime}\right)\right),
\end{aligned}
$$

with $\mathcal{N}_{0}$ chosen in such a way that $\int P_{\chi_{0}, C_{0}}\left(d \Psi^{(\leq 0)}\right)=1, Z_{0}=1$ and $R_{0, \omega}$ a matrix such that $\left\|R_{0, \omega}\left(\mathbf{k}^{\prime}\right)\right\| \leq C\left|\mathbf{k}^{\prime}\right|^{2}$. Moreover, the functionals $\mathcal{V}^{(0)},\left(A \Psi^{(\leq 0)}, T_{1} \Psi^{(\leq 0)}\right)$ and $\left(\phi, \Psi^{(\leq 0)}\right)$ are the same as $\mathcal{V}_{M},\left(A, J^{(i . r .)}\right)$ and $\left(\phi, \Psi^{(i . r .)}\right)$ in Eq.(2[21), with $\Psi^{(i . r .)}$ and $\phi^{ \pm}$rewritten 
in terms of $\Psi_{\omega}^{(\leq 0)}$ and $\phi_{\omega}^{ \pm}$; similarly $S^{(\leq 0)}(A, \phi)+B^{(0)}$ is the same as $B_{M}$ after the same rewriting, with $S^{(\leq 0)}$ collecting all the terms depending on $A$ and/or $\phi$ but not on $\Psi^{(\leq 0)}$. E.g., to be more explicit, defining $\hat{A}_{\mathbf{p}^{\prime}, \sharp}^{\omega-\omega^{\prime}}:=\hat{A}_{\mathbf{p}_{F}^{\omega}-\mathbf{p}_{F}^{\omega^{\prime}}+\mathbf{p}^{\prime}, \sharp}$ and recalling the definition of $n_{ \pm}$, i.e., $n_{ \pm}=\left(1 \pm \sigma_{3}\right) / 2$,

$$
\left(A \Psi^{(\leq 0)}, T_{1} \Psi^{(\leq 0)}\right)=\frac{1}{\left(\beta L^{2}\right)^{2}} \sum_{\omega, \omega^{\prime}, \sigma, \sharp} \sum_{\mathbf{k}^{\prime}, \mathbf{p}^{\prime}} \hat{A}_{\mathbf{p}^{\prime}, \sharp}^{\omega-\omega^{\prime}} \hat{\Psi}_{\mathbf{k}^{\prime}+\mathbf{p}^{\prime}, \sigma, \omega}^{(\leq h)+} T_{\sharp, 1}^{\omega, \omega^{\prime}}\left(\mathbf{k}^{\prime}, \mathbf{p}^{\prime}\right) \hat{\Psi}_{\mathbf{k}^{\prime}, \sigma, \omega^{\prime}}^{(\leq h)-},
$$

with $T_{\tau, 1}^{\omega, \omega^{\prime}}\left(\mathbf{k}^{\prime}, \mathbf{p}^{\prime}\right)=n_{\tau}$ and

$$
T_{j, 1}^{\omega, \omega^{\prime}}\left(\mathbf{k}^{\prime}, \mathbf{p}^{\prime}\right)=v_{0} \frac{2 i}{3} e^{i \frac{2 \pi}{3}(j-1)\left(\omega^{\prime} n_{+}-\omega n_{-}\right)}\left(\sigma_{+} e^{-i \mathbf{k}^{\prime}\left(\boldsymbol{\delta}_{j}-\boldsymbol{\delta}_{1}\right)}-\sigma_{-} e^{+i\left(\mathbf{k}^{\prime}+\mathbf{p}^{\prime}\right)\left(\boldsymbol{\delta}_{j}-\boldsymbol{\delta}_{1}\right)}\right) ;
$$

similar expressions are valid for the other functionals appearing in the exponent of Eq.(2/.34).

The integration of Eq.(2/34) is performed in an iterative fashion, justified by the infrared divergence caused by the singularity at $\mathbf{k}^{\prime}=\mathbf{0}$ of the propagator $\hat{g}_{\omega}^{(\leq 0)}\left(\mathbf{k}^{\prime}\right)$, see Eqs.(2[33),(2[.36). We define $f_{h}\left(\mathbf{k}^{\prime}\right):=\chi_{0}\left(2^{-h}\left|\mathbf{k}^{\prime}\right|\right)-\chi_{0}\left(2^{-h+1}\left|\mathbf{k}^{\prime}\right|\right)$ and rewrite

$$
\chi_{0}\left(\left|\mathbf{k}^{\prime}\right|\right)=\sum_{k=-\infty}^{0} f_{k}\left(\mathbf{k}^{\prime}\right)=: \chi_{h}\left(\mathbf{k}^{\prime}\right)+f_{h+1}\left(\mathbf{k}^{\prime}\right)+\cdots+f_{0}\left(\mathbf{k}^{\prime}\right) .
$$

The integration procedure consists in the following: we first rewrite $\chi_{0}=\chi_{-1}+f_{0}$ and correspondingly decompose the propagator $g_{\omega}^{(\leq 0)}=g_{\omega}^{(\leq-1)}+g_{\omega}^{(0)}$ and the field $\Psi_{\omega}^{(\leq 0)}=\Psi_{\omega}^{(\leq-1)}+\Psi_{\omega}^{(0)}$; next, we integrate out the "0-mode", i.e., the Grassmann field $\Psi_{\omega}^{(0)}$, and re-express the result in terms of a new effective potential on scale -1 ; then we iterate, by integrating out step by step the degrees of freedom on scale $-1,-2, \ldots, h+1$, with $h<0$. After the integration of the fields on scales $-1,-2, \ldots, h+1$, we get:

$$
\begin{aligned}
e^{\mathcal{W}_{\beta, L}(A, \phi)}= & e^{-\beta L^{2} F_{h}+S^{(\geq h)}(A, \phi)} \\
& \cdot \int P_{\chi_{h}, C_{h}}\left(d \Psi^{(\leq h)}\right) e^{\mathcal{V}^{(h)}\left(\Psi^{(\leq h)}\right)+B^{(h)}\left(A, \Psi^{(\leq h)}, \phi\right)+\left(A \Psi^{(\leq h)}, T_{h+1} \Psi^{(\leq h)}\right)+\left(\phi, Q^{(h+1)} \Psi^{(\leq h)}\right)}
\end{aligned}
$$

where $F_{h}, C_{h}, \mathcal{V}^{(h)}, B^{(h)}, T_{h}, Q^{(h)}$ will be defined recursively (in particular, $F_{h}, C_{h}$ [called $A_{h}$ in [11]], $\mathcal{V}^{(h)}, Q^{(h)}$ have already been defined in [11]) and $P_{\chi_{h}, C_{h}}\left(d \Psi^{(\leq h)}\right)$ is defined in the same way as $P_{\chi_{0}, C_{0}}\left(d \Psi^{(\leq 0)}\right)$ with $\Psi^{(\leq 0)}, \chi_{0}, C_{0, \omega}, Z_{0}, v_{0}, R_{0, \omega}$ replaced by $\Psi^{(\leq h)}, \chi_{h}, C_{h, \omega}, Z_{h}, v_{h}, R_{h, \omega}$, respectively. Moreover,

$$
\begin{aligned}
\mathcal{V}^{(h)}(\Psi)= & \sum_{n=1}^{\infty}\left(\beta L^{2}\right)^{-2 n} \sum_{\underline{\sigma}, \underline{\rho}, \underline{\underline{\omega}, \underline{\mathbf{k}}^{\prime}}}\left[\prod_{j=1}^{n} \hat{\Psi}_{\mathbf{k}_{2 j-1}^{\prime}, \sigma_{j}, \rho_{2 j-1}, \omega_{2 j-1}}^{(\leq h)+} \hat{\Psi}_{\mathbf{k}_{2 j}^{\prime}, \sigma_{j}, \rho_{2 j}, \omega_{2 j}}^{(\leq h)-}\right] . \\
& \cdot \hat{W}_{2 n ; \underline{\underline{\rho}} ; \underline{\underline{\omega}}}^{(h)}\left(\mathbf{k}_{1}^{\prime}, \ldots, \mathbf{k}_{2 n-1}^{\prime}\right) \delta\left(\sum_{j=1}^{2 n}(-1)^{j}\left(\mathbf{p}_{F}^{\omega_{j}}+\mathbf{k}_{j}^{\prime}\right)\right)
\end{aligned}
$$


and

$$
\begin{aligned}
& B^{(h)}(A, \Psi, \phi)=\sum_{n, m \geq 0}^{*}\left(\beta L^{2}\right)^{-2 n-m} \sum_{\substack{\sigma, \underline{\rho}, \sharp, \forall, \underline{\gamma}, \underline{\omega} \\
\underline{\mathbf{k}^{\prime}}, \underline{\mathbf{p}}^{\prime}}}^{*}\left[\prod_{j=1}^{n} \hat{\varphi}_{\mathbf{k}_{2 j-1}^{\prime}, \sigma_{j}, \rho_{2 j-1}, \gamma_{2 j-1}, \omega_{2 j-1}}^{+} \hat{\varphi}_{\mathbf{k}_{2 j}^{\prime}, \sigma_{j}, \rho_{2 j}, \gamma_{2 j}, \omega_{2 j}}^{-}\right] \\
& \cdot\left[\prod_{i=1}^{m} \hat{A}_{\sharp i, \mathbf{p}_{i}^{\prime}}^{\omega_{i}-\omega_{i}^{\prime}}\right] \hat{W}_{2 n, m ; \underline{\underline{\rho} \sharp ;}, \underline{\omega} ; \underline{\gamma}}^{(h)}\left(\left\{\mathbf{k}_{j}^{\prime}\right\},\left\{\mathbf{p}_{i}^{\prime}\right\}\right) \delta\left(\sum_{j=1}^{2 n}(-1)^{j+1}\left(\mathbf{k}_{j}^{\prime}+\mathbf{p}_{F}^{\omega_{j}}\right)-\sum_{i=1}^{m}\left(\mathbf{p}_{i}^{\prime}+\mathbf{p}_{F}^{\omega_{i}}-\mathbf{p}_{F}^{\omega_{i}^{\prime}}\right)\right)
\end{aligned}
$$

where: the $*^{\prime}$ 's on the sums remind the fact that only terms explicitly depending on $\hat{\varphi}_{\text {int }}^{ \pm}=$ $\hat{\Psi}^{(\leq h) \pm}$ contribute to $B^{(h)} ; \underline{\omega}=\left(\omega_{1}, \ldots, \omega_{2 n} ; \omega_{1}-\omega_{1}^{\prime}, \ldots, \omega_{m}-\omega_{m}^{\prime}\right)$.

The terms $\left(A \Psi^{(\leq h)}, T_{h+1} \Psi^{(\leq h)}\right)$ and $\left(\phi, Q^{(h+1)} \Psi^{(\leq h)}\right)$ read:

$$
\begin{aligned}
& \left(A \Psi^{(\leq h)}, T_{h+1} \Psi^{(\leq h)}\right)=\frac{1}{\left(\beta L^{2}\right)^{2}} \sum_{\substack{\omega, \omega^{\prime}, \sigma, \sharp \\
\mathbf{k}^{\prime}, \mathbf{p}^{\prime}}} \hat{A}_{\mathbf{p}^{\prime}, \sharp}^{\omega-\omega^{\prime}} \hat{\Psi}_{\mathbf{k}^{\prime}+\mathbf{p}^{\prime}, \sigma, \omega}^{(\leq h)+} T_{\sharp, h+1}^{\omega, \omega^{\prime}}\left(\mathbf{k}^{\prime}, \mathbf{p}^{\prime}\right) \hat{\Psi}_{\mathbf{k}^{\prime}, \sigma, \omega^{\prime}}^{(\leq h)-}, \\
& \left(\phi, Q^{(h+1)} \Psi^{(\leq h)}\right)=\frac{1}{\beta L^{2}} \sum_{\omega, \sigma, \mathbf{k}^{\prime}}\left(\hat{\Psi}_{\mathbf{k}^{\prime}, \sigma, \omega}^{(\leq h)+} \hat{Q}_{\mathbf{k}^{\prime}, \omega}^{(h+1)-} \hat{\phi}_{\mathbf{k}^{\prime}, \sigma, \omega}^{-}+\hat{\phi}_{\mathbf{k}^{\prime}, \sigma, \omega}^{+} \hat{Q}_{\mathbf{k}^{\prime}, \omega}^{(h+1)+} \hat{\Psi}_{\mathbf{k}^{\prime}, \sigma, \omega}^{(\leq h)-}\right),
\end{aligned}
$$

for suitable matrices $T_{\sharp, h+1}^{\omega, \omega^{\prime}}\left(\mathbf{k}^{\prime}, \mathbf{p}^{\prime}\right)$ and $\hat{Q}_{\mathbf{k}^{\prime}, \omega}^{(h+1) \pm}$, with $Q_{\mathbf{k}^{\prime}, \omega}^{(1) \pm}=1$. The iterative procedure goes on up to scale $h_{\beta}$, where $h_{\beta}$ is the largest scale such that $a_{0} \gamma^{h_{\beta}-1}<\frac{\pi}{\beta}$. The result of the last iteration is $e^{\mathcal{W}_{\beta, L}(A, \phi)}$.

\section{Localization and renormalization.}

In order to inductively prove Eq.(2,40), we rewrite

$$
\mathcal{V}^{(h)}\left(\Psi^{(\leq h)}\right)=\mathcal{L} \mathcal{V}^{(h)}\left(\Psi^{(\leq h)}\right)+\mathcal{R} \mathcal{V}^{(h)}\left(\Psi^{(\leq h)}\right)
$$

where

$$
\mathcal{L} \mathcal{V}^{(h)}\left(\Psi^{(\leq h)}\right)=\frac{1}{\beta L^{2}} \sum_{\sigma, \omega} \sum_{\mathbf{k}^{\prime}} \hat{\Psi}_{\mathbf{k}^{\prime}, \sigma, \omega}^{(\leq h)+} \hat{W}_{2 ;(\omega, \omega)}^{(h)}\left(\mathbf{k}^{\prime}\right) \hat{\Psi}_{\mathbf{k}^{\prime}, \sigma, \omega}^{(\leq h)-},
$$

and $\mathcal{R} \mathcal{V}^{(h)}$ is given by (2,41) with $\sum_{n=1}^{\infty}$ replaced by $\sum_{n=2}^{\infty}$, that is it contains only the monomials with four or more fields (note that in Eq.(2,46) the two fermionic fields have the same $\omega$-index; terms with two different quasi-particles indices are not allowed by momentum conservation, see also the remark after (3.62) of [11]). Moreover, defining

$$
\begin{aligned}
& \bar{W}_{2, \omega}^{(h)-}\left(\mathbf{k}^{\prime}\right):=\hat{W}_{2,0 ;(\omega, \omega) ;(\text { int }, \text { ext })}^{(h)}\left(\mathbf{k}^{\prime}, \mathbf{k}^{\prime}\right), \quad \bar{W}_{2, \omega}^{(h)+}\left(\mathbf{k}^{\prime}\right):=\hat{W}_{2,0 ;(\omega, \omega) ;(\text { ext }, \text { int })}^{(h)}\left(\mathbf{k}^{\prime}, \mathbf{k}^{\prime}\right), \\
& \hat{W}_{\sharp ; \omega, \omega^{\prime}}^{(h)}\left(\mathbf{k}^{\prime}, \mathbf{p}^{\prime}\right):=\sum_{\omega_{1}, \omega_{2}} \hat{W}_{2,1 ; \sharp ;\left(\omega, \omega^{\prime} ; \omega-\omega^{\prime}\right) ;(\text { int }, \text { int })}^{(h)}\left(\mathbf{k}^{\prime}+\mathbf{p}^{\prime}, \mathbf{k}^{\prime}, \mathbf{p}^{\prime}\right),
\end{aligned}
$$


we rewrite:

$$
B^{(h)}\left(A, \Psi^{(\leq h)}, \phi\right)=\mathcal{L} B^{(h)}\left(A, \Psi^{(\leq h)}, \phi\right)+\mathcal{R} B^{(h)}\left(A, \Psi^{(\leq h)}, \phi\right)
$$

where

$$
\begin{aligned}
\mathcal{L} B^{(h)}\left(A, \Psi^{(\leq h)}, \phi\right) & =\frac{1}{\beta L^{2}} \sum_{\sigma, \omega, \mathbf{k}^{\prime}}\left(\hat{\Psi}_{\mathbf{k}^{\prime}, \sigma, \omega}^{(\leq h)+} \bar{W}_{2, \omega}^{(h)-}\left(\mathbf{k}^{\prime}\right) \hat{\phi}_{\mathbf{k}^{\prime}, \sigma, \omega}^{-}+\hat{\phi}_{\mathbf{k}^{\prime}, \sigma, \omega}^{+} \bar{W}_{2, \omega}^{(h)+} \hat{\Psi}_{\mathbf{k}^{\prime}, \sigma, \omega}^{-}\right)+ \\
& +\frac{1}{\left(\beta L^{2}\right)^{2}} \sum_{\substack{\omega, \omega^{\prime} \\
\sigma, \sharp}} \sum_{\mathbf{k}^{\prime}, \mathbf{p}^{\prime}} \hat{A}_{\mathbf{p}^{\prime}, \sharp}^{\omega-\omega^{\prime}} \hat{\Psi}_{\mathbf{k}^{\prime}+\mathbf{p}^{\prime}, \sigma, \omega}^{(\leq h)+} \hat{W}_{\sharp ; \omega, \omega^{\prime}}^{(h)}\left(\mathbf{k}^{\prime}, \mathbf{p}^{\prime}\right) \hat{\Psi}_{\mathbf{k}^{\prime}, \sigma, \omega}^{(\leq h)-} .
\end{aligned}
$$

At this point we "reabsorbe" $\mathcal{L} \mathcal{V}^{(h)}$ in the fermionic gaussian integration and $\mathcal{L} B^{(h)}$ into the definition of the effective source terms:

$$
\begin{gathered}
e^{-\beta L^{2} F_{h}+S^{(\geq h)}(A, \phi)} \int P_{\chi_{h}, C_{h}}\left(d \Psi^{(\leq h)}\right) e^{\mathcal{V}^{(h)}\left(\Psi^{(\leq h)}\right)+B^{(h)}\left(A, \Psi^{(\leq h)}, \phi\right)+\left(A \Psi^{(\leq h)}, T_{h+1} \Psi^{(\leq h)}\right)+\left(\phi, Q^{(h+1)} \Psi^{(\leq h)}\right)} \\
=e^{-\beta L^{2}\left(F_{h}+e_{h}\right)+S^{(\geq h)}(A, \phi)} \int P_{\chi_{h}, \bar{C}_{h-1}\left(d \Psi^{(\leq h)}\right) e^{\mathcal{R} \mathcal{V}^{(h)}\left(\Psi^{(\leq h)}\right)}} \\
\cdot e^{\mathcal{R} B^{(h)}\left(A, \Psi^{(\leq h)}, \phi\right)+\left(A \Psi^{(\leq h)}, T_{h} \Psi^{(\leq h)}\right)+\left(\phi, Q^{(h)} \Psi^{(\leq h)}\right)}
\end{gathered}
$$

where $e_{h}$ is a suitable constant (see Eq.(3.67) of [11]) and

$$
\begin{aligned}
& \bar{C}_{h-1, \omega}\left(\mathbf{k}^{\prime}\right)=C_{h, \omega}\left(\mathbf{k}^{\prime}\right)+\chi_{h}\left(\mathbf{k}^{\prime}\right) \hat{W}_{2 ;(\omega, \omega)}^{(h)}\left(\mathbf{k}^{\prime}\right), \\
& \hat{Q}_{\mathbf{k}^{\prime}, \omega}^{(h)-}=\hat{Q}_{\mathbf{k}^{\prime}, \omega}^{(h+1)-}+\hat{W}_{2 ;(\omega, \omega)}^{(h)}\left(\mathbf{k}^{\prime}\right) \sum_{k=h+1}^{1} \hat{g}_{\omega}^{(k)} \hat{Q}_{\mathbf{k}^{\prime}, \omega}^{(k)-}, \\
& \hat{Q}_{\mathbf{k}^{\prime}, \omega}^{(h)+}=\hat{Q}_{\mathbf{k}^{\prime}, \omega}^{(h+1)+}+\left(\sum_{k=h+1}^{1} \hat{Q}_{\mathbf{k}^{\prime}, \omega}^{(k)+} \hat{g}_{\omega}^{(k)}\right) \hat{W}_{2 ;(\omega, \omega)}^{(h)}\left(\mathbf{k}^{\prime}\right), \\
& T_{\sharp, h}^{\omega, \omega^{\prime}}\left(\mathbf{k}^{\prime}, \mathbf{p}^{\prime}\right)=T_{\sharp, h+1}^{\omega, \omega^{\prime}}\left(\mathbf{k}^{\prime}, \mathbf{p}^{\prime}\right)+\hat{W}_{\sharp ; \omega, \omega^{\prime}}^{(h)}\left(\mathbf{k}^{\prime}, \mathbf{p}^{\prime}\right) .
\end{aligned}
$$

The second and third equations in (2151) can be proved as in [11], see Eqs.(3.111)-(3.113). We are now ready to perform the integration of the $\Psi^{(h)}$ field: we rewrite the Grassmann field $\Psi^{(\leq h)}$ as a sum of two independent Grassmann fields $\Psi^{(\leq h-1)}+\Psi^{(h)}$ and correspondingly we rewrite the r.h.s. of Eq.(2,50) as

$$
\begin{aligned}
& e^{-\beta L^{2}\left(F_{h}+e_{h}\right)+S^{(\geq h)}(A, \phi)} \int P_{\chi_{h-1}, C_{h-1}}\left(d \Psi^{(\leq h-1)}\right) \int P_{f_{h}, \bar{C}_{h-1}}\left(d \Psi^{(h)}\right) e^{\mathcal{R} \mathcal{V}^{(h)}\left(\Psi^{(\leq h-1)}+\Psi^{(h)}\right)} \\
& \cdot e^{\mathcal{R} B^{(h)}\left(A, \Psi^{(\leq h-1)}+\Psi^{(h)}, \phi\right)+\left(A\left(\Psi^{(\leq h-1)}+\Psi^{(h)}\right), T_{h}\left(\Psi^{(\leq h-1)}+\Psi^{(h)}\right)\right)+\left(\phi, Q^{(h)}\left(\Psi^{(\leq h-1)}+\Psi^{(h)}\right)\right)}
\end{aligned}
$$

where

$$
C_{h-1, \omega}\left(\mathbf{k}^{\prime}\right)=C_{h, \omega}\left(\mathbf{k}^{\prime}\right)+\hat{W}_{2 ;(\omega, \omega)}^{(h)}\left(\mathbf{k}^{\prime}\right)
$$


In [11] we proved (see Eqs.(3.68)-(3.69) and proof of Theorem 2 of [11]) that the single scale propagator, defined as

$$
\begin{aligned}
& \int P_{f_{h}, \bar{C}_{h-1}}\left(d \Psi^{(h)}\right) \Psi_{\mathbf{x}_{1}, \sigma_{1}, \omega_{1}}^{(h)-} \Psi_{\mathbf{x}_{2}, \sigma_{2}, \omega_{2}}^{(h)+}=\delta_{\sigma_{1}, \sigma_{2}} \delta_{\omega_{1}, \omega_{2}} g_{\omega}^{(h)}\left(\mathbf{x}_{1}-\mathbf{x}_{2}\right) \\
& g_{\omega}^{(h)}\left(\mathbf{x}_{1}-\mathbf{x}_{2}\right)=\frac{1}{\beta L^{2}} \sum_{\mathbf{k}^{\prime} \in \mathcal{B}_{\beta, L}^{\omega}} e^{-i \mathbf{k}^{\prime}\left(\mathbf{x}_{1}-\mathbf{x}_{2}\right)} f_{h}\left(\mathbf{k}^{\prime}\right)\left[\bar{A}_{h-1, \omega}\left(\mathbf{k}^{\prime}\right)\right]^{-1}
\end{aligned}
$$

can be rewritten as

$$
\begin{aligned}
g_{\omega}^{(h)}\left(\mathbf{x}_{1}-\mathbf{x}_{2}\right) & =\frac{1}{\beta L^{2}} \sum_{\mathbf{k}^{\prime} \in \mathcal{B}_{\beta, L}^{\omega}} e^{-i \mathbf{k}^{\prime}\left(\mathbf{x}_{1}-\mathbf{x}_{2}\right)} \\
\cdot \frac{f_{h}\left(\mathbf{k}^{\prime}\right)}{\bar{Z}_{h-1}\left(\mathbf{k}^{\prime}\right)} & \left(\begin{array}{cc}
-i k_{0} & \bar{v}_{h-1}\left(\mathbf{k}^{\prime}\right)\left(i k_{1}^{\prime}-\omega k_{2}^{\prime}\right) \\
\bar{v}_{h-1}\left(\mathbf{k}^{\prime}\right)\left(-i k_{1}^{\prime}-\omega k_{2}^{\prime}\right) & -i k_{0}
\end{array}\right)^{-1}\left(1+\bar{R}_{h-1, \omega}\left(\mathbf{k}^{\prime}\right)\right)^{-1}
\end{aligned}
$$

with $\bar{Z}_{h-1}, \bar{v}_{h-1}$ two functions such that (choosing $0<\theta<1$ )

$$
\begin{array}{ll}
\left.\left|\bar{Z}_{h-1}\left(\mathbf{k}^{\prime}\right)-\bar{Z}_{h}\left(\mathbf{k}^{\prime}\right)\right| \leq \text { (const. }\right)|U| 2^{\theta h}, & \bar{Z}_{0}\left(\mathbf{k}^{\prime}\right)=Z_{0}=1 \\
\left.\left|\bar{v}_{h-1}\left(\mathbf{k}^{\prime}\right)-\bar{v}_{h}\left(\mathbf{k}^{\prime}\right)\right| \mid \leq \text { (const. }\right)|U| 2^{\theta h}, & \bar{v}_{0}\left(\mathbf{k}^{\prime}\right)=v_{0}=\frac{3}{2} t
\end{array}
$$

and $\bar{R}_{h-1, \omega}$ a matrix such that $\left\|\bar{R}_{h-1, \omega}\left(\mathbf{k}^{\prime}\right)\right\| \leq$ (const.) $\left|\mathbf{k}^{\prime}\right|^{2}$ and, if $2^{h-1} \leq\left|\mathbf{k}^{\prime}\right| \leq 2^{h+1}$, $\left\|\partial_{\mathbf{k}^{\prime}}^{n} R_{h-1, \omega}\left(\mathbf{k}^{\prime}\right)\right\| \leq$ (const.) $2^{(2-n) h}$. The constants $Z_{h}:=\bar{Z}_{h}(\mathbf{0})$ and $v_{h}:=\bar{v}_{h}(\mathbf{0})$ play the role of effective wave function renormalization and Fermi velocity on scale $h$. Similarly, the local parts of the matrices $T_{\sharp, h}^{\omega, \omega^{\prime}}\left(\mathbf{k}^{\prime}, \mathbf{p}^{\prime}\right)$ play the role of effective vertices; in particular, the "relativistic" vertex functions, which represent the dominant contribution in the infrared to the kernel of the three point function, are defined as (see the subsection The three-point function below for more details):

$$
Z_{0, h}=\sum_{\tau= \pm} T_{\tau, h}^{\omega, \omega}(\mathbf{0}, \mathbf{0}), \quad Z_{1, h}=-\sigma_{2} \sum_{j=1}^{3}\left(\vec{\delta}_{j}\right)_{1} T_{j, h}^{\omega, \omega}(\mathbf{0}, \mathbf{0}), \quad Z_{2, h}=-\omega \sigma_{1} \sum_{j=1}^{3}\left(\vec{\delta}_{j}\right)_{2} T_{j, h}^{\omega, \omega}(\mathbf{0}, \mathbf{0}) .
$$

From the symmetries discussed in Appendix B and C (see also Eqs.(2[.30)-(2!.31) $), Z_{\mu, h}$, $\mu=0,1,2$, are all real, independent of $\omega$ and proportional to the identity matrix and, therefore, they can be regarded as constants, as we will do in the following; moreover, $Z_{1, h}=Z_{2, h}, Z_{0,0}=1, Z_{1,0}=Z_{2,0}=v_{0}$ and $\left|Z_{\mu, h-1}-Z_{\mu, h}\right| \leq$ (const.) $|U| 2^{\theta h}$, see Eq.(21.64) and following discussion for a proof. 
Now, going back to Eq.(2.52), if we integrate out the field on scale $h$ and define:

$$
\begin{aligned}
e^{-\beta L^{2} \bar{e}_{h-1}+S^{(\geq h-1)}(A, \phi)+\mathcal{V}^{(h-1)}\left(\Psi^{(\leq h-1)}\right)+B^{(h-1)}\left(A, \Psi^{(\leq h-1)}, \phi\right)}:= \\
=e^{S^{(\geq h)}(A, \phi)} \int P_{f_{h}, \bar{C}_{h-1}\left(d \Psi^{(h)}\right) e^{\mathcal{R} \mathcal{V}^{(h)}\left(\Psi^{(\leq h-1)}+\Psi^{(h)}\right)+\mathcal{R} B^{(h)}\left(A, \Psi^{(\leq h-1)}+\Psi^{(h)}, \phi\right)} .} \cdot e^{\left(A \Psi^{(h)}, T_{h} \Psi^{(\leq h-1)}\right)+\left(A \Psi^{(\leq h-1)}, T_{h} \Psi^{(h)}\right)+\left(A \Psi^{(h)}, T_{h} \Psi^{(h)}\right)+\left(\phi, Q^{(h)} \Psi^{(h)}\right)},
\end{aligned}
$$

we get Eq.(2,40) with $h$ replaced by $h-1$ (and $\left.F_{h-1}=F_{h}+e_{h}+\bar{e}_{h}\right)$.

The integration in Eq.(2158) can be performed by expanding in series the exponential in the r.h.s. and integrating term by term with respect to the gaussian integration $P_{f_{h}, \bar{C}_{h-1}}\left(d \Psi^{(h)}\right)$. This gives rise to an expansion for $\mathcal{W}_{\beta, L}(A, \phi)$, which can be conveniently represented in terms of Gallavotti-Nicolò trees, as described in Section 3.3 of [11] and in the next subsection.

\section{Tree expansion.}

For each $n \geq 0$ and $m \geq 2$, we introduce a family $\mathcal{T}_{h, n}^{m}$ of rooted labelled trees, defined in a way similar to the family $\mathcal{T}_{h, n}$ described in Section 3.3. of [11] (which we refer to for more details), with the following minor modifications:

1. $\mathcal{T}_{h, n}^{m}$ has $n+m$ endpoints (rather than $n$ ); $n$ of them are called normal endpoints and $m$ of them are called special endpoints; moreover, the special endpoints can be either of type $A$ or of type $\phi$. If $v$ is a normal endpoint, then, as in [11], it is associated to one of the monomials with four or more Grassmann fields contributing to $\mathcal{R}^{(0)}\left(\Psi^{\left(\leq h_{v}-1\right)}\right)$; if $v$ is a special endpoint of type $A$, then it is associated to one of the monomials contributing to $\left(A \Psi^{\left(\leq h_{v}-1\right)}, T_{h_{v}-1} \Psi^{\left(\leq h_{v}-1\right)}\right)-\left(A \Psi^{\left(\leq h_{v}-2\right)}, T_{h_{v}-1} \Psi^{\left(\leq h_{v}-2\right)}\right)$; if $v$ is a special endpoint of type $\phi$, then it is associated to one of the monomials contributing to $\left(\phi, Q^{\left(h_{v}-1\right)} \Psi^{\left(h_{v}-1\right)}\right)$.

2. Among the sets of field labels and external field labels associated to the vertex $v$, denoted by $I_{v}$ and $P_{v}$ in [11], we distinguish the field labels of type $A, \psi$ and $\phi$; we correspondingly introduce the sets $P_{v}^{A}, P_{v}^{\psi}, P_{v}^{\phi}, I_{v}^{A}, I_{v}^{\psi}$ and $I_{v}^{\phi}$. All the trees contributing to $\mathcal{W}_{\beta, L}(A, \phi)+\beta L^{2} F_{h_{\beta}}$ are characterized by the fact that $P_{v_{0}}^{\psi}=\emptyset$ and $P_{v_{0}}^{A} \cup P_{v_{0}}^{\phi} \neq \emptyset$. 
Given $\tau \in \mathcal{T}_{h, n}^{m}$, the sets $P_{v}^{\#}, \# \in\{A, \phi, \psi\}$, satisfy several constraints that depend on $\tau$ (see [11]). In particular:

a. denoting by $v_{0}$ the vertex immediately following the root on $\tau$, we have $\left|P_{v_{0}}^{\psi}\right|=0$ and $\left|P_{v_{0}}^{A}\right|+\left|P_{v_{0}}^{\phi}\right|>0$; if $v>v_{0}$, then $\left|P_{v}^{\psi}\right|>0$;

b. for any $v \in \tau$, sets of external field labels such that $\left|P_{v}\right|=\left|P_{v}^{\psi}\right|=2$ are not allowed (this follows from the definition of localization Eqs.(2[45) -(2[46) and from the choice of "reabsorbing" at each step the local part into the gaussian integration, see Eqs.(2.50), (21.52), (2.58) );

c. if $v$ is not an endpoint, then sets of external field labels such that $\left|P_{v}\right|=2$ and $\left|P_{v}^{\psi}\right|=\left|P_{v}^{\phi}\right|=1$ or such that $\left|P_{v}\right|=3,\left|P_{v}^{A}\right|=1$ and $\left|P_{v}^{\psi}\right|=2$ are not allowed (this follows from the definition of localization Eqs.(2[48)-(2[49) and from the choice of "reabsorbing" at each step the bilinear terms $\left(\phi, \bar{W}_{2, \omega}^{(h)} \Psi\right)$ into the definition of $\left(\phi, Q^{(h)} \Psi\right)$ and the "vertex" terms $\left(A \Psi, W_{\omega, \omega^{\prime}}^{(h)} \Psi\right)$ into the definition of $\left(A \Psi, T_{h-1} \Psi\right)$, see Eq.(2.51)).

As in [11], we denote by $\mathcal{P}_{\tau}$ the family of all the choices of $P_{v}^{\#}$ compatible with these constraints and by $\mathbf{P}$ the elements of $\mathcal{P}_{\tau}$. The generating functional can be expressed as a sum over trees in the following fashion (analogous to Eqs.(3.77),(3.79),(3.87),(3.88) of [11]):

$$
\mathcal{W}_{\beta, L}(A, \phi)+\beta L^{2} F_{h_{\beta}}=\sum_{n \geq 0} \sum_{m \geq 2} \sum_{h \geq h_{\beta}} \sum_{\tau \in \mathcal{T}_{h, n}^{m}} \sum_{\mathbf{P} \in \mathcal{P}_{\tau}} \sum_{T \in \mathbf{T}} \mathcal{W}^{(h)}(\tau, \mathbf{P}, T)
$$

where, as explained in [11], $\mathbf{T}$ is a suitable family of spanning trees. The contribution $\mathcal{W}^{(h)}(\tau, \mathbf{P}, T)$ can be further rewritten as

$$
\mathcal{W}^{(h)}(\tau, \mathbf{P}, T)=\int d \mathbf{x}_{v_{0}} \tilde{A}\left(P_{v_{0}}^{A}\right) \tilde{\phi}\left(P_{v_{0}}^{\phi}\right) W_{\tau, \mathbf{P}, T}^{(h)}\left(\mathbf{x}_{v_{0}}\right),
$$

where

$$
\tilde{A}\left(P_{v_{0}}^{A}\right)=\prod_{f \in P_{v_{0}}^{A}} e^{-i\left(\mathbf{p}_{F}^{\omega(f)}-\mathbf{p}_{F}^{\omega^{\prime}(f)}\right) \cdot \mathbf{x}(f)} A_{\mathbf{x}(f), \sharp(f)}^{\omega(f))}, \quad \tilde{\phi}\left(P_{v_{0}}^{\phi}\right)=\prod_{f \in P_{v_{0}}^{\phi}} e^{i \varepsilon(f) \mathbf{p}_{F}^{\omega(f)} \cdot \mathbf{x}(f)} \phi_{\mathbf{x}(f), \sigma(f), \omega(f)}^{\varepsilon(f)}
$$


and, calling $v_{i}^{*}, \ldots, v_{n}^{*}$ the endpoints of $\tau$, putting $h_{i}=h_{v_{i}^{*}}$ and denoting by $K_{v_{i}^{*}}^{\left(h_{i}\right)}\left(\mathbf{x}_{v_{i}^{*}}\right)$ the kernels associated to the endpoints,

$$
\begin{aligned}
& W_{\tau, \mathbf{P}, T}\left(\mathbf{x}_{v_{0}}\right)=\left[\prod_{i=1}^{n} K_{v_{i}^{*}}^{\left(h_{i}\right)}\left(\mathbf{x}_{v_{i}^{*}}\right)\right] . \\
& \cdot\left\{\prod_{v \text { not e.p. }} \frac{1}{s_{v} !} \int d P_{T_{v}}\left(\mathbf{t}_{v}\right) \operatorname{det} G^{h_{v}, T_{v}}\left(\mathbf{t}_{v}\right)\left[\prod_{l \in T_{v}} \delta_{\omega_{l}^{-}, \omega_{l}^{+}} \delta_{\sigma_{l}^{-}, \sigma_{l}^{+}}\left[g_{\omega_{l}}^{\left(h_{v}\right)}\left(\mathbf{x}_{l}-\mathbf{y}_{l}\right)\right]_{\rho_{l}^{-}, \rho_{l}^{+}}\right]\right\} .
\end{aligned}
$$

In the latter equation, $d P_{T}(\mathbf{t})$ is a probability measure with support on a set of $\mathbf{t}$ such that $t_{i i^{\prime}}=\mathbf{u}_{i} \cdot \mathbf{u}_{i^{\prime}}$ for some family of vectors $\mathbf{u}_{i} \in \mathbb{R}^{s}$ of unit norm. Finally $G^{h, T}(\mathbf{t})$ is a Gram matrix, whose elements are given by, see [11], Eq.(3.83):

$$
G_{i j, i^{\prime} j^{\prime}}^{h, T}=t_{i i^{\prime}} \delta_{\omega_{l}^{-}, \omega_{l}^{+}} \delta_{\sigma_{l}^{-}, \sigma_{l}^{+}}\left[g_{\omega_{l}}^{(h)}\left(\mathbf{x}_{i j}-\mathbf{y}_{i^{\prime} j^{\prime}}\right)\right]_{\rho_{l}^{-}, \rho_{l}^{+}}
$$

this matrix takes into account all the possible contractions of fields not involved in the spanning tree $T$. See [11] for more details. The effective potential $\mathcal{V}^{(h)}$ and the effective source term $B^{(h)}$ admit representations very similar to Eqs.(21.59)-(2!.60)-(2!.62), the main difference being that these are expressed as sums over trees and field labels satisfying slightly different constraints: i.e., the trees contributing to $\mathcal{V}^{(h)}$ do not have special endpoints and are associated to external field labels such that $P_{v_{0}}^{A}=P_{v_{0}}^{\phi}=\emptyset$ and $\left|P_{v_{0}}^{\psi}\right|>0$; the trees contributing to $B^{(h)}$ have at least one special endpoint, and are associated to external field labels such that $\left|P_{v_{0}}^{\psi}\right|>0$ and $\left|P_{v_{0}}^{A}\right|+\left|P_{v_{0}}^{\phi}\right|>0$.

\section{The kernels of the special endpoints of type $A$}

In order to prove Theorem 2 and Proposition 1, we will be particularly concerned with estimating the contributions with $\left|P_{v_{0}}^{A}\right|=2$ and $\left|P_{v_{0}}^{\phi}\right|=0$ or with $\left|P_{v_{0}}^{A}\right|=1$ and $\left|P_{v_{0}}^{\phi}\right|+\left|P_{v_{0}}^{\psi}\right|=$ 2. The key estimate that we preliminarily need to prove is

$$
\left\|\hat{W}_{\sharp ; \omega, \omega^{\prime}}^{(k)}\left(\mathbf{k}^{\prime}, \mathbf{p}^{\prime}\right)\right\| \leq(\text { const. })|U| 2^{\theta k},
$$

for all $k \leq 0$ and with $\theta \in(0,1)$, uniformly in $\mathbf{k}^{\prime}, \mathbf{p}^{\prime}$. Note that Eq.(2.64) implies, in particular, that the kernel of the special endpoints of type $A$ is uniformly bounded as

$$
\left\|T_{\sharp, k}^{\omega, \omega^{\prime}}\left(\mathbf{k}^{\prime}, \mathbf{p}^{\prime}\right)\right\| \leq C_{0}
$$

for all $k \leq 0$ and a suitable constant $C_{0}$, and that $\left|Z_{\mu, h-1}-Z_{\mu, h}\right| \leq$ (const.) $|U| 2^{\theta h}$, as claimed after Eq.(21.57). Let us proceed by induction: we assume the validity of Eq.(2.64) for $k>h$ 
(so that Eq.(2,65) is valid for all $k>h$ ) and prove it for $k=h$. Using the tree expansion, we can rewrite:

$\hat{W}_{\sharp ; \omega, \tilde{\omega}}^{(h)}\left(\mathbf{k}^{\prime}, \mathbf{p}^{\prime}\right)=\frac{1}{\beta L^{2}} \sum_{n \geq 1} \sum_{\tau \in \mathcal{T}_{h, n}^{1}} \sum_{\mathbf{P} \in \mathcal{P}_{\tau}}^{*} \sum_{T \in \mathbf{T}} \int d \mathbf{x}_{v_{0}} e^{i\left(\mathbf{p}_{F}^{\omega}+\mathbf{k}^{\prime}+\mathbf{p}^{\prime}\right) \mathbf{x}-i\left(\mathbf{p}_{F}^{\tilde{\omega}}+\mathbf{k}^{\prime}\right) \mathbf{y}-i\left(\mathbf{p}_{F}^{\omega}-\mathbf{p}_{F}^{\tilde{\omega}}+\mathbf{p}^{\prime}\right) \mathbf{z}} W_{\tau, \mathbf{P}, T}^{(h)}\left(\mathbf{x}_{v_{0}}\right)$

where the $*$ on the sum reminds that $P_{v_{0}}=P_{v_{0}}^{A} \cup P_{v_{0}}^{\psi}, P_{v_{0}}^{A}=\left\{f_{1}\right\}$ and $P_{v_{0}}^{\psi}=\left\{f_{2}, f_{3}\right\}$ (with $\omega\left(f_{1}\right)=\omega\left(f_{2}\right)=\omega, \omega^{\prime}\left(f_{1}\right)=\omega\left(f_{3}\right)=\tilde{\omega}, \mathbf{x}\left(f_{1}\right)=\mathbf{z}, \mathbf{x}\left(f_{2}\right)=\mathbf{x}, \mathbf{x}\left(f_{3}\right)=\mathbf{y}, \sharp\left(f_{1}\right)=\sharp$, $\varepsilon\left(f_{2}\right)=-\varepsilon\left(f_{3}\right)=-$ and $\left.\sigma\left(f_{2}\right)=\sigma\left(f_{3}\right)\right)$. Using translation invariance, the representation Eq.(2[,62) and proceeding as in the proof of Theorem 2 of [11], we get (see Eq.(3.93) of [11])

$$
\begin{aligned}
\left\|\hat{W}_{\sharp ; \omega, \tilde{\omega}}^{(h)}\left(\mathbf{k}^{\prime}, \mathbf{p}^{\prime}\right)\right\| \leq & \sum_{n \geq 1} \sum_{\tau \in \mathcal{T}_{h, n}^{1}} \sum_{\mathbf{P} \in \mathcal{P}_{\tau}}^{*} \sum_{T \in \mathbf{T}} \int \prod_{l \in T^{*}} d\left(\mathbf{x}_{l}-\mathbf{y}_{l}\right)\left[\prod_{i=1}^{n}\left|K_{v_{i}^{*}}^{\left(h_{i}\right)}\left(\mathbf{x}_{v_{i}^{*}}\right)\right|\right] . \\
& \cdot\left[\prod_{v \text { not e.p. }} \frac{1}{s_{v} !} \max _{\mathbf{t}_{v}}\left|\operatorname{det} G^{h_{v}, T_{v}}\left(\mathbf{t}_{v}\right)\right| \prod_{l \in T_{v}}\left\|g_{\omega_{l}}^{\left(h_{v}\right)}\left(\mathbf{x}_{l}-\mathbf{y}_{l}\right)\right\|\right] .
\end{aligned}
$$

The r.h.s. of this equation can be bounded dimensionally, using the scaling properties of the propagators $g_{\omega}^{\left(h_{v}\right)}(\mathbf{x})$ and of the Gram determinants $\operatorname{det} G^{h_{v}, T_{v}}\left(\mathbf{t}_{v}\right)$ (see Eqs.(3.92),(3.94) of [11]). Following the proof of Theorem 2 of [11] and using in particular Eqs.(3.94)-(3.95)(3.96), we get the analogue of Eq.(3.97) of [11], that is

$$
\begin{aligned}
& \left\|\hat{W}_{\sharp ; \omega, \tilde{\omega}}^{(h)}\left(\mathbf{k}^{\prime}, \mathbf{p}^{\prime}\right)\right\| \leq \\
& \leq \sum_{n \geq 1} \sum_{\tau \in \mathcal{T}_{h, n}^{1}} \sum_{\mathbf{P} \in \mathcal{P}_{\tau}}^{*} \sum_{T \in \mathbf{T}} C^{n}\left[\prod_{\substack{v \text { not } \\
\text { e.p. }}} \frac{1}{s_{v} !} 2^{h_{v}\left(\left(\sum_{i=1}^{s_{v}}\left|P_{v_{i}}^{\psi}\right|\right)-\left|P_{v}^{\psi}\right|-3\left(s_{v}-1\right)\right)}\right]\left[\prod_{v \text { e.p. }} C^{\frac{\left|P_{v}^{\psi}\right|}{2}} \mid U^{\frac{\left|P_{v}^{\psi}\right|}{2}-1}\right]
\end{aligned}
$$

where $p_{i}=\left|P_{v_{i}^{*}}\right|$ and $C$ is a suitable positive constant, larger than the constant $C_{0}$ appearing in Eq.(2,65) (in fact, in deriving this bound, we estimated the kernel of the special endpoint of type $A$ by using the inductive hypothesis Eq.(2,65)). The r.h.s. of this expression can be rewritten in a convenient form, using the analogues of Eqs.(3.98)-(3.100) of [11], that is (recalling that $m$ is the number of special endpoints in $\tau$-equal to 1 in the current case),

$$
\begin{aligned}
& \sum_{\substack{h \text { not } \\
\text { e.p. }}} h_{v}\left[\left(\sum_{i=1}^{s_{v}}\left|P_{v_{i}}^{\psi}\right|\right)-\left|P_{v}^{\psi}\right|\right]=h\left(\left|I_{v_{0}}^{\psi}\right|-\left|P_{v_{0}}^{\psi}\right|\right)+\sum_{\substack{h \text { not } \\
\text { e.p. }}}\left(h_{v}-h_{v^{\prime}}\right)\left(\left|I_{v}^{\psi}\right|-\left|P_{v}^{\psi}\right|\right), \\
& \sum_{\substack{h \text { not } \\
\text { e.p. }}} h_{v}\left(s_{v}-1\right)=h(n+m-1)+\sum_{\substack{h \text { not } \\
\text { e.p. }}}\left(h_{v}-h_{v^{\prime}}\right)(n(v)+m(v)-1),
\end{aligned}
$$

where: $v^{\prime}$ is the vertex immediately preceding $v$ on $\tau ; I_{v} \supseteq P_{v}$ is the set of field labels associated to $v$ (i.e., including both the internal and the external fields to $v$ ); $n(v)$ is the 
number of normal endpoints following $v$ on $\tau ; m(v)$ is the number of special endpoints following $v$ on $\tau$. Note that in the current case, where there is only one special endpoint of type $A, m(v)=\left|P_{v}^{A}\right|$ and $m=\left|P_{v_{0}}^{A}\right|$. Plugging Eq.(2!69) into Eq.(2[68) and using Eq.(3.100) of [11], we get the analogue of Eq.(3.101) of [11], that is,

$$
\begin{aligned}
\left\|\hat{W}_{\sharp ; \omega, \tilde{\omega}}^{(h)}\left(\mathbf{k}^{\prime}, \mathbf{p}^{\prime}\right)\right\| \leq & \sum_{n \geq 1} \sum_{\tau \in \mathcal{T}_{h, n}^{1}} \sum_{\mathbf{P} \in \mathcal{P}_{\tau}}^{*} \sum_{T \in \mathbf{T}} C^{n} 2^{h\left(3-\left|P_{v_{0}}^{\psi}\right|-\left|P_{v_{0}}^{A}\right|\right)} \cdot\left[\prod_{\substack{v \text { not } \\
\text { e.p. }}} \frac{1}{s_{v} !} 2^{\left(h_{v}-h_{v^{\prime}}\right)\left(3-\left|P_{v}^{\psi}\right|-\left|P_{v}^{A}\right|\right)}\right] . \\
& \cdot\left[\prod_{v \text { e.p. }} 2^{h_{v^{\prime}}\left(\left|P_{v}^{\psi}\right|+\left|P_{v}^{A}\right|-3\right)}\right]\left[\prod_{v \text { e.p. }} C^{\left.\frac{\left|P_{v}^{\psi}\right|}{2}|U|^{\frac{\left|P_{v}^{\psi}\right|}{2}-1}\right]}\right.
\end{aligned}
$$

where we used that, if $v$ is an endpoint, then $\left|I_{v}^{\psi}\right|=\left|P_{v}^{\psi}\right|$. At this point, by Eqs.(3.102)(3.103) of [11] and by the argument described after Eq.(3.103) of [11], we end up with the analogue of Eq.(3.104) of [11], that is

$$
\left\|\hat{W}_{\sharp ; \omega, \tilde{\omega}}^{(h)}\left(\mathbf{k}^{\prime}, \mathbf{p}^{\prime}\right)\right\| \leq \gamma^{h\left(3-\left|P_{v_{0}}^{\psi}\right|-\left|P_{v_{0}}^{A}\right|+\theta\right)} \sum_{n \geq 1} C^{n}|U|^{n} .
$$

Recalling that in the current case $3-\left|P_{v_{0}}^{\psi}\right|-\left|P_{v_{0}}^{A}\right|=0$, this proves the desired estimate on the kernels of the special endpoints of type A, Eq.(2164). A similar strategy also allows us to prove that

$$
T_{\sharp, h}^{\omega, \omega^{\prime}}\left(\mathbf{k}^{\prime}, \mathbf{p}^{\prime}\right)=T_{\sharp, h}^{\omega, \omega^{\prime}}(\mathbf{0}, \mathbf{0})+O\left(\left(\left|\mathbf{k}^{\prime}\right|+\left|\mathbf{p}^{\prime}\right|\right)+|U|\left(\left|\mathbf{k}^{\prime}\right|+\left|\mathbf{p}^{\prime}\right|\right)^{\theta}\right),
$$

with $\theta \in(0,1)$. Let us also recall that also the kernels $Q^{(h)}$ of the special endpoints of type $\phi$ admit a uniform bound of the form $\left\|Q^{(h)}\right\| \leq C_{0}$, see Eq.(3.114) of [11]. We are now ready to give the proof of Theorem 2 and Proposition 1.

\section{The three-point function (Proof of Theorem 2).}

The goal is to bound $\hat{G}_{2,1 ; l}^{\beta, L}(\mathbf{k}, \mathbf{p})$ at $\mathbf{k} \neq \mathbf{p}_{F}^{ \pm}, \mathbf{p} \neq \mathbf{0}$, with $\left|\mathbf{k}-\mathbf{p}_{F}^{\omega}\right|$ and $|\mathbf{p}|$ sufficiently small (and $|\mathbf{p}| \ll\left|\mathbf{k}-\mathbf{p}_{F}^{\omega}\right|$ ), for a given $\omega \in\{+,-\}$. The three-point function, by definition, using Eqs.(2!10)-(2!13)-(2:17) and Eqs.(2!.59) $-(2 ! 62)$, can be rewritten as

$$
\hat{G}_{2,1 ; l}^{\beta, L}(\mathbf{k}, \mathbf{p})=\frac{e}{\beta L^{2}} \sum_{j=1}^{3}\left(\vec{\delta}_{j}\right)_{l} \eta_{\vec{p}}^{j} \sum_{n \geq 0} \sum_{h \geq h_{\beta}} \sum_{\tau \in \mathcal{T}_{h, n}^{3}} \sum_{\mathbf{P} \in \mathcal{P}_{\tau}}^{* *} \sum_{T \in \mathbf{T}} \int d \mathbf{x}_{v_{0}} e^{i \mathbf{k}(\mathbf{x}-\mathbf{y})+i \mathbf{p}(\mathbf{x}-\mathbf{z})} W_{\tau, \mathbf{P}, T}^{(h)}\left(\mathbf{x}_{v_{0}}\right),
$$

where the $* *$ on the sum over $\mathbf{P}$ reminds that $P_{v_{0}}=P_{v_{0}}^{A} \cup P_{v_{0}}^{\phi}, P_{v_{0}}^{A}=\left\{f_{1}\right\}$ and $P_{v_{0}}^{\phi}=\left\{f_{2}, f_{3}\right\}$, with $\omega\left(f_{1}\right)=\omega^{\prime}\left(f_{1}\right)=\omega\left(f_{2}\right)=\omega\left(f_{3}\right)=\omega, \mathbf{x}\left(f_{1}\right)=\mathbf{z}, \mathbf{x}\left(f_{2}\right)=\mathbf{x}, \mathbf{x}\left(f_{3}\right)=\mathbf{y}, \sharp\left(f_{1}\right)=j$, 
$\varepsilon\left(f_{2}\right)=-\varepsilon\left(f_{3}\right)=-$ and $\sigma\left(f_{2}\right)=\sigma\left(f_{3}\right)$. The reason why all the quasi-particle indices of the external legs are equal to $\omega$ is that, by assumption, $|\mathbf{p}| \ll\left|\mathbf{k}-\mathbf{p}_{F}^{\omega}\right| \ll 1$, so that by momentum conservation all other choices of quasi-particle indices give zero contribution to $\hat{G}_{2,1 ; l}^{\beta, L}(\mathbf{k}, \mathbf{p})$. The trees contributing to $\hat{G}_{2,1 ; l}^{\beta, L}(\mathbf{k}, \mathbf{p})$ have a few more features that are worth remarking. First of all, among the three special endpoints of $\tau \in \mathcal{T}_{h, n}^{3}$, one of them is of type $A$ (let us call it $v_{A}$ and note that $f_{1} \in P_{v_{A}}^{A}$ ) and the other two are of type $\phi$ (let us call them $v_{\phi}^{+}, v_{\phi}^{-}$, with $f_{2} \in P_{v_{\phi}^{-}}^{\phi}$ and $f_{3} \in P_{v_{\phi}^{+}}^{\phi}$. Moreover, let $h_{\mathbf{k}}$ be the (negative) integer such that $2^{h_{\mathbf{k}}} \leq\left|\mathbf{k}-\mathbf{p}_{F}^{\omega}\right|<2^{h_{\mathbf{k}}+1}$, and let $|\mathbf{p}|$ be so small that $2^{h_{\mathbf{k}}-1}<\left|\mathbf{k}+\mathbf{p}-\mathbf{p}_{F}^{\omega}\right|<2^{h_{\mathbf{k}}+2}$ : then, only trees with $\left|h_{v_{\phi}^{ \pm}}-h_{\mathbf{k}}\right| \leq 1$ and with $h \leq h_{\mathbf{k}}+1$ contribute to $\hat{G}_{2,1 ; l}^{\beta, L}(\mathbf{k}, \mathbf{p})$.

Now, let us distinguish in the r.h.s. of Eq.(2.73), the contributions with $n=0$ and those with $n \geq 1$. The latter can be bounded in a way completely analogous to $\hat{W}_{\sharp ; \omega, \tilde{\omega}}^{(h)}\left(\mathbf{k}^{\prime}, \mathbf{p}^{\prime}\right)$, with the following important differences. In the current case $m=3$ and $m(v)=m^{A}(v)+m^{\phi}(v)$, with $m^{A}(v)=\left|P_{v}^{A}\right|$ (resp. $\left.m^{\phi}(v)=\left|P_{v}^{\phi}\right|\right)$ the number of special endpoints of type $A$ (resp. type $\phi$ ) following $v$ on $\tau$. Taking this into account and following the same strategy used to bound $\hat{W}_{\sharp ; \omega, \tilde{\omega}}^{(h)}$, see Eq.(2!67)-(2!.68) -(2!69) , we get the analogue of Eq.(2!70), that is we can bound the contributions with $n \geq 1$ to the three point function by

$$
\begin{aligned}
e \sum_{n \geq 1} \sum_{h \leq h_{\mathbf{k}}+1} \sum_{\tau \in \mathcal{T}_{h, n}^{3}} \sum_{\mathbf{P} \in \mathcal{P}_{\tau}}^{* *} \sum_{T \in \mathbf{T}} C^{n} 2^{h\left(3-\left|P_{v_{0}}^{\psi}\right|-\left|P_{v_{0}}^{A}\right|-\left|P_{v_{0}}^{\phi}\right|\right)} \cdot\left[\prod_{v \text { not }} \frac{1}{s_{v} !} 2^{\left(h_{v}-h_{v^{\prime}}\right)\left(3-\left|P_{v}^{\psi}\right|-\left|P_{v}^{A}\right|-\left|P_{v}^{\phi}\right|\right)}\right] . \\
\cdot\left[\prod_{v \text { e.p. }} 2^{h_{v^{\prime}}\left(\left|P_{v}^{\psi}\right|+\left|P_{v}^{A}\right|+\left|P_{v}^{\phi}\right|-3\right)}\right]\left[\prod_{v \text { e.p. }} C^{\frac{\left|P_{v}^{\psi}\right|}{2}|U|^{\left|P_{v}^{\psi}\right|}-1}\right]
\end{aligned}
$$

Now note that, if $v$ is a special endpoint of type $\phi$, then $\left|P_{v}^{\psi}\right|+\left|P_{v}^{A}\right|+\left|P_{v}^{\phi}\right|-3=-1$ and that, if $v$ is a special endpoint of type $A$, then $\left|P_{v}^{\psi}\right|+\left|P_{v}^{A}\right|+\left|P_{v}^{\phi}\right|-3=0$. Therefore, using the fact that $\left|P_{v_{0}}\right|=3$, we can rewrite Eq.(2.74) as

$$
\begin{aligned}
e 2^{-2 h_{\mathbf{k}}} \sum_{n \geq 1} \sum_{h \leq h_{\mathbf{k}}+1} \sum_{\tau \in \mathcal{T}_{h, n}^{3}} \sum_{\mathbf{P} \in \mathcal{P}_{\tau}}^{* *} \sum_{T \in \mathbf{T}} C^{n} \cdot\left[\prod_{\substack{\text { not. } \\
\text { e.p. }}} \frac{1}{s_{v} !} 2^{\left(h_{v}-h_{v^{\prime}}\right)\left(3-\left|P_{v}\right|\right)}\right] . \\
\cdot\left[\prod_{\substack{\text { normal } \\
\text { e.p. }}} 2^{h_{v^{\prime}}\left(\left|P_{v}\right|-3\right)}\right]\left[\prod_{v \text { e.p. }} C^{\frac{\left|P_{v}^{\psi}\right|}{2}}|U|^{\frac{\mid P_{v}^{\psi}}{2}-1}\right] .
\end{aligned}
$$

The only potentially dangerous contributions to Eq.(2.75) are those coming from a vertex $v$ that is not an endpoint and such that $\left|P_{v}\right|=3$. By construction, such vertex has necessarily $\left|P_{v}^{A}\right|=\left|P_{v}^{\psi}\right|=\left|P_{v}^{\phi}\right|=1$; on the other hand, by momentum conservation, $0<h_{v}-h_{v^{\prime}} \leq 2$, 
simply because $|\mathbf{p}| \ll\left|\mathbf{k}-\mathbf{p}_{F}^{\omega}\right| \ll 1$ and, therefore, the quasi-momenta associated to the external fields $\phi$ and $\Psi$ have essentially the same momentum scale (i.e., the two scales differ at most by 1); in conclusion, the overall contribution coming from a vertex that is not an endpoint and such that $\left|P_{v}\right|=3$ can be bounded by an $O(1)$ constant and gives no trouble. Therefore, Eq.(2.75) implies that the overall contribution to $\hat{G}_{2,1 ; l}^{\beta, L}(\mathbf{k}, \mathbf{p})$ coming from trees with $n \geq 1$ can be bounded by (const.) $|U| 2^{(-2+\theta) h_{\mathbf{k}}}$. We are left with the contributions coming from the trees with $n=0$, which read (defining $\mathbf{k}^{\prime}=\mathbf{k}-\mathbf{p}_{F}^{\omega}$ )

$$
e \sum_{h, h^{\prime}=h_{\mathbf{k}}}^{h_{\mathbf{k}}+1} Q_{\mathbf{k}^{\prime}+\mathbf{p}, \omega}^{(h)+} \hat{g}_{\omega}^{(h)}\left(\mathbf{k}^{\prime}+\mathbf{p}\right)\left[\sum_{j=1}^{3}\left(\vec{\delta}_{j}\right)_{l} \eta_{\vec{p}}^{j} T_{j, h}^{\omega, \omega}\left(\mathbf{k}^{\prime}, \mathbf{p}\right)\right] g_{\omega}^{\left(h^{\prime}\right)}\left(\mathbf{k}^{\prime}\right) Q_{\mathbf{k}^{\prime}, \omega}^{\left(h^{\prime}\right)-},
$$

where $\bar{h}:=\max \left\{h, h^{\prime}\right\}$. Using Eq.(2164), as well as the estimates on $Q^{(h)}$ and on the two-point Schwinger function proved in [11], see Eqs.(3.114),(3.120)-(3.122) of [11], we can rewrite Eq.(2.76) as

$$
e\left(\hat{S}(\mathbf{k}+\mathbf{p})\left[\sum_{j=1}^{3}\left(\vec{\delta}_{j}\right){ }_{l} \eta_{\vec{p}}^{j} T_{j, h_{\mathbf{k}}}^{\omega, \omega}\left(\mathbf{k}^{\prime}, \mathbf{p}\right)\right] \hat{S}(\mathbf{k})\right)\left(1+O\left(|U| 2^{\theta h_{\mathbf{k}}}\right)\right),
$$

for some $\theta \in(0,1)$. Moreover, using Eq.(2/.72), we can further rewrite Eq.(21.77) as

$$
e\left(\hat{S}(\mathbf{k}+\mathbf{p}) Z_{l, h_{\mathbf{k}}} \Gamma_{l}\left(\vec{p}_{F}^{\omega}, \overrightarrow{0}\right) \hat{S}(\mathbf{k})\right)\left(1+O\left(2^{h_{\mathbf{k}}}\right)+O\left(|U| 2^{\theta h_{\mathbf{k}}}\right)\right),
$$

where the vertex functions $Z_{l, h}$ were defined in Eq.(21.57) and we remind the reader that $\Gamma_{1}\left(\vec{p}_{F}^{\omega}, \overrightarrow{0}\right)=-\sigma_{2}$ and $\Gamma_{2}\left(\vec{p}_{F}^{\omega}, \overrightarrow{0}\right)=-\omega \sigma_{1}$. Theorem 2 is an immediate corollary of the previous estimates and, in particular, of Eqs.(21.75) $-(2.78)$. Using the fact that $\left|Z_{l, h}-Z_{l, h-1}\right| \leq$ (const.) $|U| 2^{\theta h}$, we also find that the constants $Z_{\mu}$ in the statement of Theorem 2 coincide with the infrared limit of the running coupling constants $Z_{\mu, h}$, i.e., $Z_{\mu}=\lim _{h \rightarrow-\infty} Z_{\mu, h}$.

\section{The response function (Proof of Proposition 1).}

In order to prove Proposition 1, we start by deriving bounds on the current-current response function $K_{l m}^{\beta, L}(\mathbf{x}-\mathbf{y})$ at distinct space-time points, $\mathbf{x} \neq \mathbf{y}$, which can be expressed in terms of the tree expansion as follows:

$$
K_{l m}^{\beta, L}(\mathbf{x}-\mathbf{y})=e^{2} v_{0}^{2} \sum_{j, j^{\prime}=1}^{3}\left(\vec{\delta}_{j}\right)_{l}\left(\vec{\delta}_{j^{\prime}}\right)_{m} \sum_{n \geq 0} \sum_{h \geq h_{\beta}} \sum_{\tau \in \mathcal{T}_{h, n}^{2}} \sum_{\mathbf{P} \in \mathcal{P}_{\tau}}^{* * *} \sum_{T \in \mathbf{T}} \int d \mathbf{x}_{v_{0}}^{*}\left(H_{j, j^{\prime}} * W_{\tau, \mathbf{P}, T}^{(h)}\right)\left(\mathbf{x}_{v_{0}}\right),
$$


where the $* * *$ on the sum over $\mathbf{P}$ reminds that $P_{v_{0}}=P_{v_{0}}^{A}=\left\{f_{1}, f_{2}\right\}$, with $\omega\left(f_{1}\right)-\omega^{\prime}\left(f_{1}\right)=$ $\omega\left(f_{2}\right)-\omega^{\prime}\left(f_{2}\right)=0, \mathbf{x}\left(f_{1}\right)=\mathbf{x}, \mathbf{x}\left(f_{2}\right)=\mathbf{y}, \mathbf{x}\left(f_{3}\right)=\mathbf{y}, \sharp\left(f_{1}\right)=j$ and $\sharp\left(f_{2}\right)=j^{\prime}$; moreover, the * over $d \mathbf{x}_{v_{0}}$ reminds that we are integrating over all the variables in $\mathbf{x}_{v_{0}}$ but $\mathbf{x}$ and $\mathbf{y}$, which are fixed (and distinct); finally, $H_{j, j^{\prime}}$ is the Fourier transform of $\eta_{\vec{p}}^{j} \eta_{-\vec{p}}^{j^{\prime}}$ and $\left(H_{j, j^{\prime}} * W_{\tau, \mathbf{P}, T}^{(h)}\right)$ denotes the convolutions between $H_{j, j^{\prime}}$ and $W_{\tau, \mathbf{P}, T}$. The trees contributing to $K_{l m}^{\beta, L}$ have two special endpoints of type $A$; we call them $v_{1}$ and $v_{2}$, we denote by $v^{*}$ the rightest vertex such that $v^{*} \leq v_{1}, v_{2}$ with respect to the partial order induced by the tree and by $h^{*}$ its scale. Proceeding as in the previous subsection, we distinguish the contributions to $K_{l m}^{\beta, L}$ coming from trees of order $n=0$ or $n \geq 1$; we denote the two by $K_{l m}^{(0)}$ and $K_{l m}^{(1)}$, respectively. The latter can be bounded by (using a notation analogous to the one used in Eq.(2.67))

$$
\begin{gathered}
\left\|K_{l m}^{(1)}(\mathbf{x}-\mathbf{y})\right\| \leq e^{2} v_{0}^{2} \sum_{j, j^{\prime}=1}^{3}\left\|H_{j, j^{\prime}}\right\|_{L_{1}} \sum_{n \geq 1} \sum_{h \geq h_{\beta}} \sum_{\tau \in \mathcal{T}_{h, n}^{2}} \sum_{\mathbf{P} \in \mathcal{P}_{\tau}}^{* * *} \sum_{T \in \mathbf{T}} \int \prod_{l \in T^{*}}^{*} d\left(\mathbf{x}_{l}-\mathbf{y}_{l}\right) \cdot(2,80) \\
\cdot\left[\prod_{i=1}^{n}\left|K_{v_{i}^{*}}^{\left(h_{h^{*}}\right)}\left(\mathbf{x}_{v_{i}^{*}}\right)\right|\right]\left[\prod_{v \text { not e.p. }} \frac{1}{s_{v} !} \max _{\mathbf{t}_{v}}\left|\operatorname{det} G^{h_{v}, T_{v}}\left(\mathbf{t}_{v}\right)\right| \prod_{l \in T_{v}}\left\|g_{\omega_{l}}^{\left(h_{v}\right)}\left(\mathbf{x}_{l}-\mathbf{y}_{l}\right)\right\|\right]
\end{gathered}
$$

where $\prod_{l \in T^{*}}^{*}$ is the product over all lines of the (modified) spanning tree $T^{*}$, but one line belonging to the subtree $T_{\mathbf{x}, \mathbf{y}} \subset T^{*}$ connecting $\mathbf{x}$ with $\mathbf{y}$ and contained into the cluster $v^{*}$ but not in any smaller one; let us call $\bar{l}$ this special line. Eq.(2180) can be bounded in a way analogous to Eq.(2167), with the important difference that the $L_{1}$ norm of the propagator associated to $\bar{l}$ is replaced by its $L_{\infty}$ norm, which has a factor $2^{3 h^{*}}$ more as compared to the $L_{1}$ norm; moreover, in order to take into account the decay between $\mathbf{x}$ and $\mathbf{y}$, we can extract a factor $\frac{C_{N}^{n}}{1+\left(2^{h^{*}}|\mathbf{x}-\mathbf{y}|\right)^{N}}$ from the product of the propagators in the spanning tree (here $N \geq 1$ and $C_{N}$ is a suitable positive constant); we are still left with an expression that can be estimated in the same way as Eq.(2!67), thus leading to the upper bound

$$
\begin{aligned}
& \left\|K_{l m}^{(1)}(\mathbf{x}-\mathbf{y})\right\| \leq e^{2} v_{0}^{2} \sum_{n \geq 1} \sum_{h \geq h_{\beta}} \sum_{\tau \in \mathcal{T}_{h, n}^{2}} \sum_{\mathbf{P} \in \mathcal{P}_{\tau}}^{* * *} \sum_{T \in \mathbf{T}} C^{n} 2^{h\left(3-\left|P_{v_{0}}\right|\right)} \cdot 2^{3 h^{*}} \cdot \frac{C_{N}^{n}}{1+\left(2^{h^{*}}|\mathbf{x}-\mathbf{y}|\right)^{N}} \cdot \\
& \cdot\left[\prod_{\substack{v \text { not. } \\
\text { e.p. }}} \frac{1}{s_{v} !} 2^{\left(h_{v}-h_{v^{\prime}}\right)\left(3-\left|P_{v}\right|\right)}\right] \cdot\left[\prod_{v \text { e.p. }} 2^{h_{v^{\prime}}\left(\left|P_{v}\right|-3\right)}\right]\left[\prod_{v \text { e.p. }} C^{\left.\frac{\left|P_{v}^{\psi}\right|}{2}|U|^{\frac{\left|P_{v}^{\psi}\right|}{2}-1}\right]}\right.
\end{aligned}
$$

Proceeding as in the previous subsections, we see that this can be further bounded as

$$
\left\|K_{l m}^{(1)}(\mathbf{x}-\mathbf{y})\right\| \leq e^{2} \sum_{n \geq 1} \sum_{h \geq h_{\beta}} \sum_{h^{*} \geq h} C^{n}|U|^{n} 2^{h(1+\theta)} 2^{3 h^{*}} \frac{C_{N}^{n}}{1+\left(2^{h^{*}}|\mathbf{x}-\mathbf{y}|\right)^{N}} .
$$


Now, noting that, by construction, $h \geq h^{*}$ and exchanging the order of summation over $h$ and $h^{*}$, we get (picking $N=5$ and $|U|$ small enough)

$$
\left\|K_{l m}^{(1)}(\mathbf{x}-\mathbf{y})\right\| \leq(\text { const. }) e^{2}|U| \sum_{h^{*} \geq h_{\beta}} \frac{2^{h^{*}(4+\theta)}}{1+\left(2^{h^{*}}|\mathbf{x}-\mathbf{y}|\right)^{5}},
$$

which implies

$$
\| K_{l m}^{(1)}(\mathbf{x}-\mathbf{y})|| \leq \text { (const.) } e^{2}|U| \frac{1}{1+|\mathbf{x}-\mathbf{y}|^{4+\theta}}
$$

and, therefore, in the limit $\beta, L \rightarrow \infty$ (which exists by the uniformity of the bounds and by the term by term convergence of the series, see Appendix D of [11] for more details), the Fourier transform of $K^{(1)}$ is continuous and continuously differentiable for all $\mathbf{p} \in \mathbb{R} \times \mathcal{B}$ (in particular at $\mathbf{p}=\mathbf{0})$.

We are now left with the contributions to the response function coming from the trees with $n=0$, which read

$$
\begin{aligned}
& K_{l m}^{(0)}(\mathbf{x}-\mathbf{y})=-2 e^{2} v_{0}^{2} \sum_{j, j^{\prime}=1}^{3}\left(\vec{\delta}_{j}\right)_{l}\left(\vec{\delta}_{j^{\prime}}\right)_{m} \sum_{h, h^{\prime} \geq h_{\beta}} \sum_{\omega, \omega^{\prime}} \int \frac{d \mathbf{k}^{\prime} d \mathbf{p}^{\prime}}{(2 \pi)^{2}|\mathcal{B}|^{2}} e^{i\left(\mathbf{p}_{F}^{\omega}-\mathbf{p}_{F}^{\omega^{\prime}}+\mathbf{p}^{\prime}\right)(\mathbf{x}-\mathbf{y})} . \\
& \cdot \eta_{\vec{p}_{F}^{\omega}-p_{F}^{\omega^{\prime}}+\vec{p}^{\prime}} \eta_{-\left(\vec{p}_{F}^{j^{\prime}}-\bar{p}_{F}^{\omega^{\prime}}+\vec{p}^{\prime}\right)} \operatorname{Tr}\left\{\hat{g}_{\omega}^{(h)}\left(\mathbf{k}^{\prime}+\mathbf{p}^{\prime}\right) T_{j, h}^{\omega, \omega^{\prime}}\left(\mathbf{k}^{\prime}, \mathbf{p}^{\prime}\right) \hat{g}_{\omega^{\prime}}^{\left(h^{\prime}\right)}\left(\mathbf{k}^{\prime}\right) T_{j^{\prime}, \bar{h}}^{\omega^{\prime}, \omega}\left(\mathbf{k}^{\prime}+\mathbf{p}^{\prime},-\mathbf{p}^{\prime}\right)\right\},
\end{aligned}
$$

where $\bar{h}=\max \left\{h, h^{\prime}\right\}$ and the factor 2 in the r.h.s. takes into account the summation over the spin degrees of freedom. It is important to notice that the integral in the latter expression can be rewritten as $e^{i\left(\mathbf{p}_{F}^{\omega}-\mathbf{p}_{F}^{\omega^{\prime}}\right)(\mathbf{x}-\mathbf{y})} f_{h, h^{\prime}}^{\omega, \omega^{\prime} ; j j^{\prime}}(\mathbf{x}-\mathbf{y})$, with $f_{h, h^{\prime}}^{\omega, \omega^{\prime} ; j, j^{\prime}}(\mathbf{x}-\mathbf{y})$ a function admitting the dimensional bound:

$$
\left|\partial_{\mathbf{x}}^{n} f_{h, h^{\prime}}^{\omega, \omega^{\prime} ; j j^{\prime}}(\mathbf{x})\right| \leq c_{N} 2^{2\left(h+h^{\prime}\right)} \frac{2^{n \cdot \max \left\{h, h^{\prime}\right\}}}{1+\left(2^{\max \left\{h, h^{\prime}\right\}}|\mathbf{x}|\right)^{N}},
$$

for all $N \geq 0$. Eq.(2185) implies that the contributions to $K^{(0)}(\mathbf{x}-\mathbf{y})$ with $\omega \neq \omega^{\prime}$ can be rewritten as $e^{i\left(\mathbf{p}_{F}^{\omega}-\mathbf{p}_{F}^{-\omega}\right)(\mathbf{x}-\mathbf{y})} F_{\omega,-\omega}(\mathbf{x}-\mathbf{y})$, with

$$
\left|\partial_{\mathbf{x}}^{n} F_{\omega,-\omega}(\mathbf{x})\right| \leq e^{2} c_{N} \sum_{h, h^{\prime}} 2^{2\left(h+h^{\prime}\right)} \frac{2^{n \cdot \max \left\{h, h^{\prime}\right\}}}{1+\left(2^{\max \left\{h, h^{\prime}\right\}}|\mathbf{x}|\right)^{N}} \leq e^{2} \frac{c_{N}^{\prime}}{1+|\mathbf{x}|^{4+n}},
$$

for all $N \geq 4+n$. This implies, in particular, that we can rewrite

$$
\begin{aligned}
& K_{l m}^{(0)}(\mathbf{x}-\mathbf{y})=-2 e^{2} v_{0}^{2} \sum_{j, j^{\prime}=1}^{3}\left(\vec{\delta}_{j}\right)_{l}\left(\vec{\delta}_{j^{\prime}}\right)_{m} \sum_{h, h^{\prime} \geq h_{\beta}} \sum_{\omega} \int \frac{d \mathbf{k}^{\prime} d \mathbf{p}}{(2 \pi)^{2}|\mathcal{B}|^{2}} e^{i \mathbf{p}(\mathbf{x}-\mathbf{y})} \eta_{\vec{p}}^{j} \eta_{-\vec{p}^{\prime}}^{j^{\prime}} . \\
& \cdot \operatorname{Tr}\left\{\hat{g}_{\omega}^{(h)}\left(\mathbf{k}^{\prime}+\mathbf{p}\right) T_{j, h}^{\omega, \omega}\left(\mathbf{k}^{\prime}, \mathbf{p}\right) \hat{g}_{\omega}^{\left(h^{\prime}\right)}\left(\mathbf{k}^{\prime}\right) T_{j^{\prime}, \bar{h}}^{\omega, \omega}\left(\mathbf{k}^{\prime}+\mathbf{p},-\mathbf{p}\right)\right\}+H_{l m}^{(0)}(\mathbf{x}-\mathbf{y}),
\end{aligned}
$$


where the Fourier transform of $H^{(0)}(\mathbf{x}-\mathbf{y})$ is continuously differentiable in a neighborhood of $\mathbf{p}=\mathbf{0}$. Using Eq.(2.72) and the definition of $Z_{l, h}$, we can further rewrite this expression as

$$
\begin{aligned}
& K_{l m}^{(0)}(\mathbf{x}-\mathbf{y})=-2 e^{2} v_{0}^{2} \sum_{h, h^{\prime} \geq h_{\beta}} \sum_{\omega} Z_{l, h} Z_{m, h^{\prime}} \int \frac{d \mathbf{k}^{\prime} d \mathbf{p}}{(2 \pi)^{2}|\mathcal{B}|^{2}} e^{i \mathbf{p}(\mathbf{x}-\mathbf{y})} \eta_{\vec{p}}^{j} \eta_{-\vec{p}}^{j^{\prime}} \cdot \\
& \cdot \operatorname{Tr}\left\{\hat{g}_{\omega}^{(h)}\left(\mathbf{k}^{\prime}+\mathbf{p}\right) \Gamma_{l}\left(\vec{p}_{F}^{\omega}, \overrightarrow{0}\right) \hat{g}_{\omega}^{\left(h^{\prime}\right)}\left(\mathbf{k}^{\prime}\right) \Gamma_{m}\left(\vec{p}_{F}^{\omega}, \overrightarrow{0}\right)\right\}+\bar{H}_{l m}^{(0)}(\mathbf{x}-\mathbf{y})
\end{aligned}
$$

where the Fourier transform of $\bar{H}^{(0)}(\mathbf{x}-\mathbf{y})$ is continuously differentiable in a neighborhood of $\mathbf{p}=\mathbf{0}$. Finally, rewriting $Z_{l, h}=Z_{l}+O\left(|U| 2^{\theta h}\right)$, using the expression of the two-point function in terms of a sum of single scale propagators (see Eq.(3.121) of [11]) and using the definition of $\vec{\Gamma}(\vec{k}, \vec{p})$ in Eq.(11.39), we get

$$
\begin{aligned}
K_{l m}^{(0)}(\mathbf{x}-\mathbf{y})= & -2 e^{2} v_{0}^{2} Z_{l} Z_{m} \int \frac{d \mathbf{k} d \mathbf{p}}{(2 \pi)^{2}|\mathcal{B}|^{2}} e^{i \mathbf{p}(\mathbf{x}-\mathbf{y})} \eta_{\vec{p}}^{j} \eta_{-\vec{p}}^{j^{\prime}} \operatorname{Tr}\left\{\hat{S}(\mathbf{k}+\mathbf{p}) \Gamma_{l}(\vec{k}, \overrightarrow{0}) \hat{S}(\mathbf{k}) \Gamma_{m}(\vec{k}, \overrightarrow{0})\right\}+ \\
& +\widetilde{H}_{l m}^{(0)}(\mathbf{x}-\mathbf{y}),
\end{aligned}
$$

where the Fourier transform of $\widetilde{H}^{(0)}(\mathbf{x}-\mathbf{y})$ is continuously differentiable in a neighborhood of $\mathbf{p}=\mathbf{0}$. Combining Eq.(2187) with the explicit expression Eq.(11.42) for the two point function and with the bounds derived above on $K^{(1)}$, we finally obtain the statement of Proposition 1 (the parity property of $R(\mathbf{p})$ stated in item 3 of Proposition 1 easily follows from the symmetry properties listed in Appendix B and C).

\section{Appendix A: The conductivity of the non interacting system}

In this Appendix we prove Eq.(11.40), with $\left.\sigma_{i j}\right|_{U=0}$ given by Eq.(11.38):

$$
\left.\sigma_{i j}\right|_{U=0}=\frac{2}{3 \sqrt{3}} \frac{2 e^{2} v_{0}^{2}}{\hbar} \lim _{p_{0} \rightarrow 0^{+}} \int \frac{d k_{0}}{2 \pi} \int_{\mathcal{B}} \frac{d \vec{k}}{|\mathcal{B}|} \operatorname{Tr}\left\{\frac{S_{0}\left(\mathbf{k}+\left(p_{0}, 0\right)\right)-S_{0}(\mathbf{k})}{p_{0}} \Gamma_{i}(\vec{k}, \overrightarrow{0}) S_{0}(\mathbf{k}) \Gamma_{j}(\vec{k}, \overrightarrow{0})\right\},
$$

where $|\mathcal{B}|=8 \pi^{2} /(3 \sqrt{3})$ and

$$
\Gamma_{i}(\vec{k}, \overrightarrow{0})=\left(\begin{array}{cc}
0 & a_{i}(\vec{k}) \\
a_{i}^{*}(\vec{k}) & 0
\end{array}\right),
$$

with $a_{1}(\vec{k})=\frac{2 i}{3}\left[1-e^{i \frac{3}{2} k_{1}} \cos \left(\frac{\sqrt{3}}{2} k_{2}\right)\right]$ and $a_{2}(\vec{k})=\frac{2}{\sqrt{3}} e^{i \frac{3}{2} k_{1}} \sin \left(\frac{\sqrt{3}}{2} k_{2}\right)$.

Let $\varepsilon>0$ be a small number, independent of $p_{0}$, to be eventually sent to zero. In the integral to be evaluated, we distinguish between the region where $|\Omega(\vec{k})| \geq \varepsilon$ and the region 
where $|\Omega(\vec{k})| \leq \varepsilon$ :

$$
\begin{aligned}
\left.\sigma_{i j}\right|_{U=0}= & \frac{2}{3 \sqrt{3}} \frac{2 e^{2} v_{0}^{2}}{\hbar} \lim _{\varepsilon \rightarrow 0} \lim _{p_{0} \rightarrow 0^{+}} \int \frac{d k_{0}}{2 \pi} \int_{\mathcal{B}} \frac{d \vec{k}}{|\mathcal{B}|} \operatorname{Tr}\left\{\frac{S_{0}\left(\mathbf{k}+\left(p_{0}, 0\right)\right)-S_{0}(\mathbf{k})}{p_{0}} \Gamma_{i}(\vec{k}, \overrightarrow{0}) .\right. \\
& \left.\cdot S_{0}(\mathbf{k}) \Gamma_{j}(\vec{k}, \overrightarrow{0})\right\} \cdot[\chi(|\Omega(\vec{k})| \geq \varepsilon)+\chi(|\Omega(\vec{k})| \leq \varepsilon)]
\end{aligned}
$$

The integral associated to the region $\chi(|\Omega(\vec{k})| \geq \varepsilon)$ is uniformly convergent as $p_{0} \rightarrow 0^{+}$: therefore, we can exchange the integral with the limit and check that the integral of the limit is zero (simply because the integrand is odd in $k_{0}$ ). Next, in the integral associated to the region $\chi(|\Omega(\vec{k})| \leq \varepsilon)$ we rewrite the propagator as the relativistic propagator plus a correction (similarly, we rewrite $\Gamma_{i}$ as its relativistic limit plus a correction). The corrections are associated to absolutely convergent integrals, uniformly in $p_{0}$ as $p_{0} \rightarrow 0^{+}$, and one can easily check that their contribution after having taken $\varepsilon \rightarrow 0$ is equal to zero. We are left with (after having changed variables and having included a further factor 2 coming from the summation over the two Fermi points):

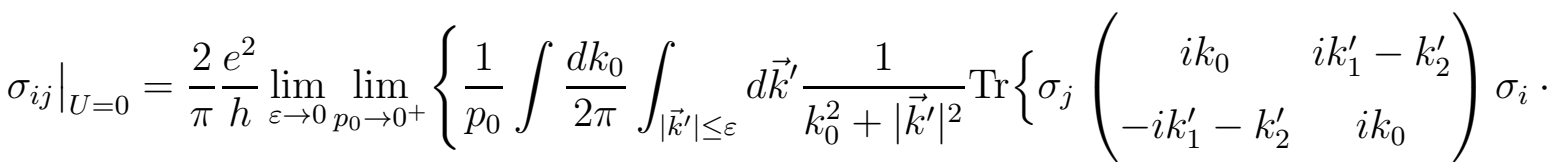

$$
\begin{aligned}
& \left.\left.\cdot\left[\frac{1}{\left(k_{0}+p_{0}\right)^{2}+\left|\vec{k}^{\prime}\right|^{2}}\left(\begin{array}{cc}
i\left(k_{0}+p_{0}\right) & i k_{1}^{\prime}-k_{2}^{\prime} \\
-i k_{1}^{\prime}-k_{2}^{\prime} & i\left(k_{0}+p_{0}\right)
\end{array}\right)-\frac{1}{k_{0}^{2}+\left|\vec{k}^{\prime}\right|^{2}}\left(\begin{array}{cc}
i k_{0} & i k_{1}^{\prime}-k_{2}^{\prime} \\
-i k_{1}^{\prime}-k_{2}^{\prime} & i k_{0}
\end{array}\right)\right]\right\}\right\},
\end{aligned}
$$

where $\sigma_{i}, i=1,2$, are the first two Pauli matrices. Now, if $i \neq j$, the r.h.s. of this equation is equal to

$$
\pm \frac{2}{\pi} \frac{e^{2}}{h} \lim _{\varepsilon \rightarrow 0} \lim _{p_{0} \rightarrow 0^{+}} \frac{1}{p_{0}} \int \frac{d k_{0}}{2 \pi} \int_{\left|\overrightarrow{k^{\prime}}\right| \leq \varepsilon} d \vec{k}^{\prime} \frac{4 k_{1}^{\prime} k_{2}^{\prime}}{k_{0}^{2}+\left|\overrightarrow{k^{\prime}}\right|^{2}}\left(\frac{1}{\left(k_{0}+p_{0}\right)^{2}+\left|\overrightarrow{k^{\prime}}\right|^{2}}-\frac{1}{k_{0}^{2}+\left|\overrightarrow{k^{\prime}}\right|^{2}}\right),
$$

which is zero by the symmetry under the exchange $\vec{k}^{\prime} \longleftrightarrow-\vec{k}^{\prime}$. If, on the contrary, $i=j$, we get:

$$
\begin{aligned}
\left.\sigma_{i i}\right|_{U=0}= & \frac{2}{\pi} \frac{e^{2}}{h} \lim _{\varepsilon \rightarrow 0} \lim _{p_{0} \rightarrow 0^{+}} \frac{1}{p_{0}} \int \frac{d k_{0}}{2 \pi} \int_{\left|\overrightarrow{k^{\prime}}\right| \leq \varepsilon} d \vec{k}^{\prime} \frac{1}{k_{0}^{2}+\left|\overrightarrow{k^{\prime}}\right|^{2}}\left[\left(\frac{2 k_{0}^{2}}{k_{0}^{2}+\left|\overrightarrow{k^{\prime}}\right|^{2}}-\frac{2 k_{0}\left(k_{0}+p_{0}\right)}{\left(k_{0}+p_{0}\right)^{2}+\left|\overrightarrow{k^{\prime}}\right|^{2}}\right)+\right. \\
& \left.+(-1)^{i}\left[2\left(k_{1}^{\prime}\right)^{2}-2\left(k_{2}^{\prime}\right)^{2}\right]\left(\frac{1}{k_{0}^{2}+\left|\overrightarrow{k^{\prime}}\right|^{2}}-\frac{1}{\left(k_{0}+p_{0}\right)^{2}+\left|\vec{k}^{\prime}\right|^{2}}\right)\right] .
\end{aligned}
$$

Now, the terms in the integral proportional to $2\left(k_{1}^{\prime}\right)^{2}-2\left(k_{2}^{\prime}\right)^{2}$ are zero by the symmetry under the exchange $k_{1}^{\prime} \longleftrightarrow k_{2}^{\prime}$. Therefore, we are left with:

$$
\left.\sigma_{i i}\right|_{U=0}=\frac{2}{\pi} \frac{e^{2}}{h} \lim _{\varepsilon \rightarrow 0} \lim _{p_{0} \rightarrow 0^{+}} \frac{1}{p_{0}} \int \frac{d k_{0}}{2 \pi} \int_{\left|\overrightarrow{k^{\prime}}\right| \leq \varepsilon} d \vec{k}^{\prime} \frac{1}{k_{0}^{2}+\left|\overrightarrow{k^{\prime}}\right|^{2}}\left(\frac{2 k_{0}^{2}}{k_{0}^{2}+\left|\overrightarrow{k^{\prime}}\right|^{2}}-\frac{2 k_{0}\left(k_{0}+p_{0}\right)}{\left(k_{0}+p_{0}\right)^{2}+\left|\overrightarrow{k^{\prime}}\right|^{2}}\right),
$$


that is

$$
\left.\sigma_{i i}\right|_{U=0}=8 \frac{e^{2}}{h} \lim _{\varepsilon \rightarrow 0} \lim _{p_{0} \rightarrow 0^{+}} \frac{1}{p_{0}} \int_{0}^{\varepsilon} d k \cdot k \int \frac{d k_{0}}{2 \pi}\left(\frac{k_{0}^{2}}{\left(k_{0}^{2}+k^{2}\right)^{2}}-\frac{k_{0}\left(k_{0}+p_{0}\right)}{\left[\left(k_{0}+p_{0}\right)^{2}+k^{2}\right] \cdot\left[k_{0}^{2}+k^{2}\right]}\right) .
$$

The integral in $k_{0}$ can be evaluated by residues to give:

$$
\begin{aligned}
& \int \frac{d k_{0}}{2 \pi i}\left[\frac{k_{0}^{2}}{\left(k_{0}^{2}+k^{2}\right)^{2}}-\frac{k_{0}\left(k_{0}+p_{0}\right)}{\left[\left(k_{0}+p_{0}\right)^{2}+k^{2}\right] \cdot\left[k_{0}^{2}+k^{2}\right]}\right]=\partial_{k_{0}}\left[\frac{k_{0}^{2}}{\left(k_{0}+i k\right)^{2}}\right]_{k_{0}=i k}- \\
& {\left[\frac{k_{0}\left(k_{0}+p_{0}\right)}{\left[\left(k_{0}+p_{0}\right)^{2}+k^{2}\right] \cdot\left[k_{0}+i k\right]}\right]_{k_{0}=i k}-\left[\frac{k_{0}\left(k_{0}+p_{0}\right)}{\left[k_{0}+p_{0}+i k\right] \cdot\left[k_{0}^{2}+k^{2}\right]}\right]_{k_{0}+p_{0}=i k},}
\end{aligned}
$$

that is

$$
\int \frac{d k_{0}}{2 \pi}\left[\frac{k_{0}^{2}}{\left(k_{0}^{2}+k^{2}\right)^{2}}-\frac{k_{0}\left(k_{0}+p_{0}\right)}{\left[\left(k_{0}+p_{0}\right)^{2}+k^{2}\right] \cdot\left[k_{0}^{2}+k^{2}\right]}\right]=\frac{p_{0}^{2}}{4 k\left(p_{0}^{2}+4 k^{2}\right)} .
$$

Plugging (A.9) into (A.7) gives

$$
\left.\sigma_{i i}\right|_{U=0}=8 \frac{e^{2}}{h} \lim _{\varepsilon \rightarrow 0} \lim _{p_{0} \rightarrow 0^{+}} \frac{p_{0}}{16} \int_{0}^{\varepsilon} d k \frac{1}{k^{2}+p_{0}^{2} / 4}=\frac{e^{2}}{h} \lim _{\varepsilon \rightarrow 0} \lim _{p_{0} \rightarrow 0^{+}} \arctan \left(2 \varepsilon / p_{0}\right)=\frac{e^{2}}{h} \frac{\pi}{2},
$$

which is the desired result.

\section{Appendix B: Symmetry transformations}

In the Appendix we collect some symmetry properties of the fermionic action, i.e., some transformation of the fermionic fields and of the external sources that leave separately invariant both the gaussian fermionic integration $P(d \Psi)$ and the interactions $\mathcal{V}(\Psi),(\Psi, \phi)$, $(A, J)$. These symmetries will be also preserved by the multiscale integration and, therefore, they will allow us to exclude the presence of possibly dangerous terms in the effective action at scale $h$, see Section 2 and Appendix C. In the following, we denote by $\sigma_{1}, \sigma_{2}, \sigma_{3}$ the standard Pauli matrices and we use the follwoing convention for the Fourier transform of the $A$ field: $A_{\mathbf{x}, \sharp}=\left(\beta L^{2}\right)^{-1} \sum_{\mathbf{p} \in \overline{\mathcal{B}}_{\beta, L}} e^{-i \mathbf{p x}} \hat{A}_{\mathbf{p}, \sharp}$, where $\overline{\mathcal{B}}_{\beta, L}=2 \pi \beta^{-1} \mathbb{Z} \times \mathcal{B}_{L}$ and $\sharp \in\{+,-, 1,2,3\}$.

Lemma B.1 For any choice of $M, \beta, L$, the fermionic Gaussian integration $P(d \Psi)$, the interaction $\mathcal{V}(\Psi)$ and the source terms $(\Psi, \phi),(A, J)$, defined in Eqs. (2.3)-(2.7), are separately invariant under the following transformations (here $\gamma \in\{$ ext, int $\}$ and $\hat{\varphi}_{\mathbf{k}, \sigma, i n t}=\hat{\Psi}_{\mathbf{k}, \sigma}$, while $\hat{\varphi}_{\mathbf{k}, \sigma, e x t}=\hat{\phi}_{\mathbf{k}, \sigma}$; whenever this will not create ambiguities, we shall drop the labels $\sigma$ and $\gamma$, i.e., we shall use $\hat{\varphi}_{\mathbf{k}}^{ \pm}$as a shorthand for $\left.\hat{\varphi}_{\mathbf{k}, \sigma, \gamma}^{ \pm}\right)$: 
(1) Spin flip: $\hat{\varphi}_{\mathbf{k}, \sigma, \gamma}^{\varepsilon} \leftrightarrow \hat{\varphi}_{\mathbf{k},-\sigma, \gamma}^{\varepsilon}$;

(2) Global U(1): $\hat{\varphi}_{\mathbf{k}, \sigma, \gamma}^{\varepsilon} \rightarrow \mathrm{e}^{i \varepsilon \alpha_{\sigma}} \hat{\varphi}_{\mathbf{k}, \sigma, \gamma}^{\varepsilon}$, with $\alpha_{\sigma} \in \mathbb{R}$ independent of $\mathbf{k}$;

(3) $\underline{\operatorname{Spin} S O(2)}:\left(\begin{array}{l}\hat{\varphi}_{\mathbf{k}, \uparrow, \cdot, \gamma}^{\epsilon} \\ \hat{\varphi}_{\mathbf{k}, \downarrow, \gamma}^{\epsilon}\end{array}\right) \rightarrow e^{-i \theta \sigma_{2}}\left(\begin{array}{c}\hat{\varphi}_{\mathbf{k}, \uparrow, ;, \gamma}^{\epsilon} \\ \hat{\varphi}_{\mathbf{k}, \downarrow, \cdot, \gamma}^{\epsilon}\end{array}\right)$, with $\theta \in \mathbb{T}=\mathbb{R} / 2 \pi \mathbb{Z}$ independent of $\mathbf{k}$;

(4) Discrete rotations: $\quad \hat{\varphi}_{\mathbf{k}}^{-} \rightarrow e^{-i \vec{k}\left(\vec{\delta}_{3}-\vec{\delta}_{1}\right) \frac{\sigma_{3}}{2}} \hat{\varphi}_{T \mathbf{k}}^{-}, \quad \hat{\varphi}_{\mathbf{k}}^{+} \rightarrow \hat{\varphi}_{T \mathbf{k}}^{+} e^{i \vec{k}\left(\vec{\delta}_{3}-\vec{\delta}_{1}\right) \frac{\sigma_{3}}{2}}, \quad \hat{A}_{\mathbf{p}, \pm} \rightarrow$ $\hat{A}_{T \mathbf{p}, \pm} e^{\mp i \frac{\vec{p}}{2}\left(\vec{\delta}_{3}-\vec{\delta}_{1}\right)}$ and $\hat{A}_{\mathbf{p}, j} \rightarrow \hat{A}_{T \mathbf{p}, j+1} e^{-i \frac{\vec{p}}{2}\left(\vec{\delta}_{3}-\vec{\delta}_{1}\right)}$, with $T \mathbf{k}=\left(k_{0}, e^{-i \frac{2 \pi}{3} \sigma_{2}} \vec{k}\right)$;

(5) Complex conjugation: $\hat{\varphi}_{\mathbf{k}}^{\varepsilon} \rightarrow \hat{\varphi}_{-\mathbf{k}}^{\varepsilon}, \hat{A}_{\mathbf{p}, \pm} \rightarrow \hat{A}_{-\mathbf{p}, \pm}, \hat{A}_{\mathbf{p}, j} \rightarrow-\hat{A}_{-\mathbf{p}, j}$ and $c \rightarrow c^{*}$, where $c$ is generic constant appearing in $P(d \Psi)$, in $\mathcal{V}(\Psi)$ or in $(A, J)$;

(6.a) Horizontal reflections: $\hat{\varphi}_{\mathbf{k}}^{-} \rightarrow \sigma_{1} \hat{\varphi}_{R_{h} \mathbf{k}}^{-}, \hat{\varphi}_{\mathbf{k}}^{+} \rightarrow \hat{\varphi}_{R_{h} \mathbf{k}}^{+} \sigma_{1}, \hat{A}_{\mathbf{p}, \pm} \rightarrow \hat{A}_{R_{h} \mathbf{p}, \mp}$ and $\hat{A}_{\mathbf{p}, j} \rightarrow$ $-\hat{A}_{R_{h} \mathbf{p}, r_{h} j} e^{-i \vec{p}\left(\vec{\delta}_{j}-\vec{\delta}_{1}\right)}$, with $R_{h} \mathbf{k}=\left(k_{0},-k_{1}, k_{2}\right)$ and $r_{h} 1=1, r_{h} 2=3, r_{h} 3=2$;

(6.b) Vertical reflections: $\hat{\varphi}_{\mathbf{k}}^{\varepsilon} \rightarrow \hat{\varphi}_{R_{v} \mathbf{k}}^{\varepsilon}, \hat{A}_{\mathbf{p}, \pm} \rightarrow \hat{A}_{R_{v} \mathbf{p}, \pm}$ and $\hat{A}_{\mathbf{p}, j} \rightarrow \hat{A}_{R_{v} \mathbf{p}, r_{v} j}$, with $R_{v} \mathbf{k}=$ $\left(k_{0}, k_{1},-k_{2}\right)$ and $r_{v} 1=1, r_{v} 2=3, r_{v} 3=2$;

(7) Particle-hole: $\hat{\varphi}_{\mathbf{k}}^{-} \rightarrow i \hat{\varphi}_{P \mathbf{k}}^{+, T}, \hat{\varphi}_{\mathbf{k}}^{+} \rightarrow i \hat{\varphi}_{P \mathbf{k}}^{-, T}, \hat{A}_{\mathbf{p}, \pm} \rightarrow \hat{A}_{-P \mathbf{p}, \pm}$ and $\hat{A}_{\mathbf{p}, j} \rightarrow-\hat{A}_{-P \mathbf{p}, j}$, with $P \mathbf{k}=\left(k_{0},-k_{1},-k_{2}\right)$;

(8) Inversion: $\hat{\Psi}_{\mathbf{k}, \sigma}^{-} \rightarrow-i \sigma_{3} \hat{\Psi}_{I \mathbf{k}, \sigma}^{-}, \hat{\Psi}_{\mathbf{k}, \sigma}^{+} \rightarrow-i \hat{\Psi}_{I \mathbf{k}, \sigma}^{+} \sigma_{3}, \hat{\phi}_{\mathbf{k}, \sigma}^{-} \rightarrow i \sigma_{3} \hat{\phi}_{I \mathbf{k}, \sigma}^{-}, \hat{\phi}_{\mathbf{k}, \sigma}^{+} \rightarrow i \hat{\phi}_{I \mathbf{k}, \sigma}^{+} \sigma_{3}$, $\hat{A}_{\mathbf{p}, \pm} \rightarrow-\hat{A}_{I \mathbf{p}, \pm}$ and $\hat{A}_{\mathbf{p}, j} \rightarrow \hat{A}_{I \mathbf{p}, j}$, with I $\mathbf{k}=\left(-k_{0}, k_{1}, k_{2}\right)$.

Proof. The proof of the fact that $P(d \Psi)$, and $\mathcal{V}(\Psi)$ are separately invariant under the transformations of the $\Psi$ fields has already been discussed in Section 3.1 of [11]. The fact that $(\Psi, \phi)=\left(\beta L^{2}\right)^{-1} \sum_{\mathbf{k}, \sigma}\left(\hat{\Psi}_{\mathbf{k}, \sigma}^{+} \hat{\phi}_{\mathbf{k}, \sigma}^{-}+\hat{\phi}_{\mathbf{k}, \sigma}^{+} \hat{\Psi}_{\mathbf{k}, \sigma}^{-}\right)$is invariant is apparent from the definitions. Therefore, here we are left with proving only the invariance of the term $(A, J)$ under the transformations (4) to (8) of the list above. In order to verify these symmetries, it is convenient to rewrite the source term in Fourier space:

$$
\begin{aligned}
(A, J) & =\frac{e}{\left(\beta L^{2}\right)^{2}} \sum_{\substack{\mathbf{p} \in \overline{\mathcal{B}}_{\beta, L} \\
\mathbf{k} \in \mathcal{B}_{\beta, L}^{*}}} \sum_{\tau= \pm} \hat{A}_{\mathbf{p}, \tau} \hat{\Psi}_{\mathbf{k}+\mathbf{p}, \sigma}^{+} n_{\tau} \hat{\Psi}_{\mathbf{k}, \sigma}^{-}+ \\
& +\frac{e v_{0}}{\left(\beta L^{2}\right)^{2}} \sum_{\substack{\mathbf{p} \in \overline{\mathcal{B}}_{\beta, L} \\
\mathbf{k} \in \mathcal{D}_{\beta, L}}} \sum_{j=1,2,3} \hat{A}_{\mathbf{p}, j} \hat{\Psi}_{\mathbf{k}+\mathbf{p}, \sigma}^{+}\left(i \sigma_{+} e^{-i \mathbf{k}\left(\boldsymbol{\delta}_{j}-\boldsymbol{\delta}_{1}\right)}-i \sigma_{-} e^{i(\mathbf{k}+\mathbf{p})\left(\boldsymbol{\delta}_{j}-\boldsymbol{\delta}_{1}\right)}\right) \hat{\Psi}_{\mathbf{k}, \sigma}^{-},
\end{aligned}
$$


where it is implicit that the terms in the sums with $\mathbf{k}+\mathbf{p} \notin \mathcal{B}_{\beta, L}^{*}$ should be put equal to zero.

Symmetry (4). The term

$$
(*):=\sum_{\mathbf{k}, \mathbf{p}, \tau} \hat{A}_{\mathbf{p}, \tau} \hat{\Psi}_{\mathbf{k}+\mathbf{p}, \sigma}^{+} n_{\tau} \hat{\Psi}_{\mathbf{k}, \sigma}^{-}
$$

in the first line of Eq. (B,1) is changed under (4) as:

$$
(*) \rightarrow \sum_{\mathbf{k}, \mathbf{p}, \tau} \hat{A}_{T \mathbf{p}, \tau} e^{-i \tau \frac{\vec{p}}{2}\left(\vec{\delta}_{3}-\vec{\delta}_{1}\right)} \hat{\Psi}_{T(\mathbf{k}+\mathbf{p}), \sigma}^{+}\left[e^{i(\vec{k}+\vec{p})\left(\vec{\delta}_{3}-\vec{\delta}_{1}\right) \frac{\sigma_{3}}{2}} n_{\tau} e^{-i \vec{k}\left(\vec{\delta}_{3}-\vec{\delta}_{1}\right) \frac{\sigma_{3}}{2}}\right] \hat{\Psi}_{\mathbf{k}, \sigma}^{-} .
$$

Using the definition of $n_{ \pm}$, we find that

$$
\left[e^{i(\vec{k}+\vec{p})\left(\vec{\delta}_{3}-\vec{\delta}_{1}\right) \frac{\sigma_{3}}{2}} n_{\tau} e^{-i \vec{k}\left(\vec{\delta}_{3}-\vec{\delta}_{1}\right) \frac{\sigma_{3}}{2}}\right]=e^{i \vec{p}\left(\vec{\delta}_{3}-\vec{\delta}_{1}\right) \frac{\sigma_{3}}{2}} n_{\tau}=e^{i \tau \frac{\vec{p}}{2}\left(\vec{\delta}_{3}-\vec{\delta}_{1}\right)} n_{\tau}
$$

Plugging this identity into Eq. $(\mathrm{B}, 3)$ we see that $(*)$ is invariant under (4). Similarly, the term

$$
(* *):=\sum_{\mathbf{k}, \mathbf{p}, j} \hat{A}_{\mathbf{p}, j} \hat{\Psi}_{\mathbf{k}+\mathbf{p}, \sigma}^{+}\left(i \sigma_{+} e^{-i \mathbf{k}\left(\boldsymbol{\delta}_{j}-\boldsymbol{\delta}_{1}\right)}-i \sigma_{-} e^{i(\mathbf{k}+\mathbf{p})\left(\boldsymbol{\delta}_{j}-\boldsymbol{\delta}_{1}\right)}\right) \hat{\Psi}_{\mathbf{k}, \sigma}^{-}
$$

in the second line of Eq.(B⿺辶1) is changed under (4) as:

$$
\begin{aligned}
(* *) \rightarrow \sum_{\mathbf{k}, \mathbf{p}, j} & \hat{A}_{T \mathbf{p}, j+1} e^{-i \frac{\vec{p}}{2}\left(\vec{\delta}_{3}-\vec{\delta}_{1}\right)} . \\
& \cdot \hat{\Psi}_{T(\mathbf{k}+\mathbf{p}), \sigma}^{+}\left[e^{i(\mathbf{k}+\mathbf{p})\left(\boldsymbol{\delta}_{3}-\boldsymbol{\delta}_{1}\right) \frac{\sigma_{3}}{2}}\left(i \sigma_{+} e^{-i \mathbf{k}\left(\boldsymbol{\delta}_{j}-\boldsymbol{\delta}_{1}\right)}-i \sigma_{-} e^{i(\mathbf{k}+\mathbf{p})\left(\boldsymbol{\delta}_{j}-\boldsymbol{\delta}_{1}\right)}\right) e^{-i \mathbf{k}\left(\boldsymbol{\delta}_{3}-\boldsymbol{\delta}_{1}\right)}\right] \hat{\Psi}_{\mathbf{k}, \sigma}^{-} .
\end{aligned}
$$

Using the definition of $\sigma_{ \pm}$, we find that $e^{i(\mathbf{k}+\mathbf{p})\left(\boldsymbol{\delta}_{3}-\boldsymbol{\delta}_{1}\right) \frac{\sigma_{3}}{2}} \sigma_{ \pm} e^{-i \mathbf{k}\left(\boldsymbol{\delta}_{3}-\boldsymbol{\delta}_{1}\right)}=\sigma_{ \pm} e^{ \pm i\left(\mathbf{k}+\frac{\mathbf{p}}{2}\right)\left(\boldsymbol{\delta}_{3}-\boldsymbol{\delta}_{1}\right)}$ and, therefore,

$$
\begin{aligned}
{\left[e ^ { i ( \mathbf { k } + \mathbf { p } ) ( \boldsymbol { \delta } _ { 3 } - \boldsymbol { \delta } _ { 1 } ) \frac { \sigma _ { 3 } } { 2 } } \left(i \sigma_{+} e^{-i \mathbf{k}\left(\boldsymbol{\delta}_{j}-\boldsymbol{\delta}_{1}\right)}-\right.\right.} & \left.\left.i \sigma_{-} e^{i(\mathbf{k}+\mathbf{p})\left(\boldsymbol{\delta}_{j}-\boldsymbol{\delta}_{1}\right)}\right) e^{-i \mathbf{k}\left(\boldsymbol{\delta}_{3}-\boldsymbol{\delta}_{1}\right)}\right]= \\
& =e^{i \frac{\mathbf{p}}{2}\left(\boldsymbol{\delta}_{3}-\boldsymbol{\delta}_{1}\right)}\left(i \sigma_{+} e^{-i \mathbf{k}\left(\boldsymbol{\delta}_{j}-\boldsymbol{\delta}_{3}\right)}-i \sigma_{-} e^{i(\mathbf{k}+\mathbf{p})\left(\boldsymbol{\delta}_{j}-\boldsymbol{\delta}_{3}\right)}\right) .
\end{aligned}
$$

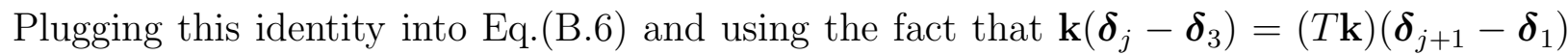
we see that also $(* *)$ is invariant under $(4)$.

Symmetry (5). The term $(*)$ is changed under (5) as:

$$
(*) \rightarrow \sum_{\mathbf{k}, \mathbf{p}, \tau} \hat{A}_{-\mathbf{p}, \tau} \hat{\Psi}_{-(\mathbf{k}+\mathbf{p}), \sigma}^{+} n_{\tau} \hat{\Psi}_{-\mathbf{k}, \sigma}^{-},
$$

which is the same as $(*)$. Similarly, the term $(* *)$ is changed under $(5)$ as:

$$
(* *) \rightarrow \sum_{\mathbf{k}, \mathbf{p}, j}\left(-\hat{A}_{-\mathbf{p}, j}\right) \hat{\Psi}_{-(\mathbf{k}+\mathbf{p}), \sigma}^{+}\left(-i \sigma_{+} e^{+i \mathbf{k}\left(\boldsymbol{\delta}_{j}-\boldsymbol{\delta}_{1}\right)}+i \sigma_{-} e^{-i(\mathbf{k}+\mathbf{p})\left(\boldsymbol{\delta}_{j}-\boldsymbol{\delta}_{1}\right)}\right) \hat{\Psi}_{-\mathbf{k}, \sigma}^{-}
$$


which is the same as $(* *)$.

Symmetry (6.a). The term (*) is changed under (6.a) as:

$$
(*) \rightarrow \sum_{\mathbf{k}, \mathbf{p}, \tau} \hat{A}_{R_{h} \mathbf{p},-\tau} \hat{\Psi}_{R_{h}(\mathbf{k}+\mathbf{p}), \sigma}^{+} \sigma_{1} n_{\tau} \sigma_{1} \hat{\Psi}_{R_{h} \mathbf{k}, \sigma}^{-}
$$

Using the fact that $\sigma_{1} n_{\tau} \sigma_{1}=n_{-\tau}$ we see that this term is invariant under (5). The term $(* *)$ is changed under (6.a) as:

$(* *) \rightarrow \sum_{\mathbf{k}, \mathbf{p}, j}\left(-\hat{A}_{R_{h} \mathbf{p}, r_{h} j}\right) e^{-i \mathbf{p}\left(\boldsymbol{\delta}_{j}-\boldsymbol{\delta}_{1}\right)} \hat{\Psi}_{R_{h}(\mathbf{k}+\mathbf{p}), \sigma}^{+}\left[\sigma_{1}\left(i \sigma_{+} e^{-i \mathbf{k}\left(\boldsymbol{\delta}_{j}-\boldsymbol{\delta}_{1}\right)}-i \sigma_{-} e^{+i(\mathbf{k}+\mathbf{p})\left(\boldsymbol{\delta}_{j}-\boldsymbol{\delta}_{1}\right)}\right) \sigma_{1}\right] \hat{\Psi}_{R_{h} \mathbf{k}, \sigma}^{-}$

where

$$
\left[\sigma_{1}\left(i \sigma_{+} e^{-i \mathbf{k}\left(\boldsymbol{\delta}_{j}-\boldsymbol{\delta}_{1}\right)}-i \sigma_{-} e^{+i(\mathbf{k}+\mathbf{p})\left(\boldsymbol{\delta}_{j}-\boldsymbol{\delta}_{1}\right)}\right) \sigma_{1}\right]=-e^{i \mathbf{p}\left(\boldsymbol{\delta}_{j}-\boldsymbol{\delta}_{1}\right)}\left(i \sigma_{+} e^{i \mathbf{k}\left(\boldsymbol{\delta}_{j}-\boldsymbol{\delta}_{1}\right)}-i \sigma_{-} e^{-i(\mathbf{k}+\mathbf{p})\left(\boldsymbol{\delta}_{j}-\boldsymbol{\delta}_{1}\right)}\right) .
$$

Using this identity and the fact that $\mathbf{k}\left(\boldsymbol{\delta}_{j}-\boldsymbol{\delta}_{1}\right)=-\left(R_{h} \mathbf{k}\right)\left(\boldsymbol{\delta}_{r_{h} j}-\boldsymbol{\delta}_{1}\right)$, we see that $(* *)$ is invariant under (6.a).

Symmetry (6.b). The term (*) is changed under (6.b) as:

$$
(*) \rightarrow \sum_{\mathbf{k}, \mathbf{p}, \tau} \hat{A}_{R_{v} \mathbf{p}, \tau} \hat{\Psi}_{R_{v}(\mathbf{k}+\mathbf{p}), \sigma}^{+} n_{\tau} \hat{\Psi}_{R_{v} \mathbf{k}, \sigma}^{-},
$$

which is obviously the same as $(*)$. The term $(* *)$ is changed under (6.b) as:

$$
(* *) \rightarrow \sum_{\mathbf{k}, \mathbf{p}, j} \hat{A}_{R_{v} \mathbf{p}, r_{v} j} \hat{\Psi}_{R_{v}(\mathbf{k}+\mathbf{p}), \sigma}^{+}\left(i \sigma_{+} e^{-i \mathbf{k}\left(\boldsymbol{\delta}_{j}-\boldsymbol{\delta}_{1}\right)}-i \sigma_{-} e^{+i(\mathbf{k}+\mathbf{p})\left(\boldsymbol{\delta}_{j}-\boldsymbol{\delta}_{1}\right)}\right) \hat{\Psi}_{R_{v} \mathbf{k}, \sigma}^{-} .
$$

Using the fact that $\mathbf{k}\left(\boldsymbol{\delta}_{j}-\boldsymbol{\delta}_{1}\right)=\left(R_{v} \mathbf{k}\right)\left(\boldsymbol{\delta}_{r_{v}}-\boldsymbol{\delta}_{1}\right)$ we see that also (**) is invariant under (6.b).

Symmetry (7). The term $(*)$ is changed under (7) as:

$$
(*) \rightarrow-\sum_{\mathbf{k}, \mathbf{p}, \tau} \hat{A}_{-P \mathbf{p}, \tau} \hat{\Psi}_{P(\mathbf{k}+\mathbf{p}), \sigma}^{-, T} n_{\tau} \hat{\Psi}_{P \mathbf{k}, \sigma}^{-}=\sum_{\mathbf{k}, \mathbf{p}, \tau} \hat{A}_{-P \mathbf{p}, \tau} \hat{\Psi}_{P \mathbf{k}, \sigma}^{+} n_{\tau} \hat{\Psi}_{P(\mathbf{k}+\mathbf{p}), \sigma}^{-}
$$

which is the same as $(*)$. The term $(* *)$ is changed under $(7)$ as:

$$
\begin{aligned}
& (* *) \rightarrow \sum_{\mathbf{k}, \mathbf{p}, j} \hat{A}_{-P \mathbf{p}, j} \hat{\Psi}_{P(\mathbf{k}+\mathbf{p}), \sigma}^{-, T}\left(i \sigma_{+} e^{-i \mathbf{k}\left(\boldsymbol{\delta}_{j}-\boldsymbol{\delta}_{1}\right)}-i \sigma_{-} e^{+i(\mathbf{k}+\mathbf{p})\left(\boldsymbol{\delta}_{j}-\boldsymbol{\delta}_{1}\right)}\right) \hat{\Psi}_{P \mathbf{k}, \sigma}^{+, T}= \\
& =\sum_{\mathbf{k}, \mathbf{p}, j} \hat{A}_{-P \mathbf{p}, j} \hat{\Psi}_{P \mathbf{k}, \sigma}^{+}\left(i \sigma_{+} e^{i(\mathbf{k}+\mathbf{p})\left(\boldsymbol{\delta}_{j}-\boldsymbol{\delta}_{1}\right)}-i \sigma_{-} e^{-i \mathbf{k}\left(\boldsymbol{\delta}_{j}-\boldsymbol{\delta}_{1}\right)}\right) \hat{\Psi}_{P(\mathbf{k}+\mathbf{p}), \sigma}^{-}
\end{aligned}
$$


Using the fact that $\mathbf{k}\left(\boldsymbol{\delta}_{j}-\boldsymbol{\delta}_{1}\right)=-(P \mathbf{k})\left(\boldsymbol{\delta}_{r_{v}}-\boldsymbol{\delta}_{1}\right)$ we see that also $(* *)$ is invariant under $(7)$.

Symmetry (8). The term (*) is changed under (8) as:

$$
(*) \rightarrow \sum_{\mathbf{k}, \mathbf{p}, \tau} \hat{A}_{I \mathbf{p}, \tau} \hat{\Psi}_{I(\mathbf{k}+\mathbf{p}), \sigma}^{+}\left[\sigma_{3} n_{\tau} \sigma_{3}\right] \hat{\Psi}_{I \mathbf{k}, \sigma}^{-},
$$

which is the same as $(*)$. The term $(* *)$ is changed under $(8)$ as:

$$
(* *) \rightarrow-\sum_{\mathbf{k}, \mathbf{p}, j} \hat{A}_{I \mathbf{p}, j} \hat{\Psi}_{I(\mathbf{k}+\mathbf{p}), \sigma}^{+}\left[\sigma_{3}\left(i \sigma_{+} e^{-i \mathbf{k}\left(\boldsymbol{\delta}_{j}-\boldsymbol{\delta}_{1}\right)}-i \sigma_{-} e^{+i(\mathbf{k}+\mathbf{p})\left(\boldsymbol{\delta}_{j}-\boldsymbol{\delta}_{1}\right)}\right) \sigma_{3}\right] \hat{\Psi}_{I \mathbf{k}, \sigma}^{-}
$$

Using the fact that $\sigma_{3} \sigma_{ \pm} \sigma_{3}=-\sigma_{ \pm}$and that $\mathbf{k}\left(\boldsymbol{\delta}_{j}-\boldsymbol{\delta}_{1}\right)=(I \mathbf{k})\left(\boldsymbol{\delta}_{j}-\boldsymbol{\delta}_{1}\right)$ we see that also $(* *)$ is invariant under $(8)$.

\section{Appendix C: Symmetry properties of the kernels}

In this appendix we prove Eqs.(2/27)-(2/31). We start by studying the symmetries of the kernel quadratic in the external field $A$ and by proving Eqs.(2[2]27)-(2,29). Next we investigate the symmetries of the kernel quadratic in the fermionic fields $\Psi$ and linear in the external field $A$ and prove Eqs.(2,30)-(2,31).

The AA kernel. We consider the term quadratic in the external fields $A_{\sharp}$ in the r.h.s. of Eq.(22,25) , which has the form (neglecting the dependence on the label $M$, defining $\hat{W}_{\sharp, b}(\mathbf{p}):=$ $\hat{W}_{0,2 ;(\sharp, b)}(\mathbf{p},-\mathbf{p})$ and assuming, without loss of generality, that $\left.\hat{W}_{\sharp, b}(\mathbf{p})=\hat{W}_{b, \sharp}(-\mathbf{p})\right)$ :

$$
\frac{1}{\beta L^{2}} \sum_{\mathbf{p}}\left[\sum_{\tau, \tau^{\prime}=+,-} \hat{A}_{\mathbf{p}, \tau} \hat{W}_{\tau, \tau^{\prime}}(\mathbf{p}) \hat{A}_{-\mathbf{p}, \tau^{\prime}}+2 \sum_{\substack{\tau= \pm, j=1,2,3}} \hat{A}_{\mathbf{p}, \tau} \hat{W}_{\tau, j}(\mathbf{p}) \hat{A}_{-\mathbf{p}, j}+\sum_{j, j^{\prime}=1,2,3} \hat{A}_{\mathbf{p}, j} \hat{W}_{j, j^{\prime}}(\mathbf{p}) \hat{A}_{-\mathbf{p}, j^{\prime}}\right]
$$

which must be invariant under the symmetry transformations listed in Appendix B. Using symmetries (4)-(8) of Appendix $[\mathrm{B}$, we find that:

$$
\begin{aligned}
\hat{W}_{\tau, \tau^{\prime}}(\mathbf{p}) & =\hat{W}_{\tau, \tau^{\prime}}(T \mathbf{p}) e^{i \frac{\mathbf{p}}{2}\left(\boldsymbol{\delta}_{3}-\boldsymbol{\delta}_{1}\right)\left(\tau-\tau^{\prime}\right)}=\hat{W}_{\tau, \tau^{\prime}}^{*}(-\mathbf{p})= \\
& =\hat{W}_{-\tau,-\tau^{\prime}}\left(R_{h} \mathbf{p}\right)=\hat{W}_{\tau, \tau^{\prime}}\left(R_{v} \mathbf{p}\right)=\hat{W}_{\tau, \tau^{\prime}}(I \mathbf{p}), \\
\hat{W}_{\tau, j}(\mathbf{p}) & =\hat{W}_{\tau, j+1}(T \mathbf{p}) e^{i \frac{\mathbf{p}}{2}\left(\boldsymbol{\delta}_{3}-\boldsymbol{\delta}_{1}\right)(\tau-1)}=-\hat{W}_{\tau, j}^{*}(-\mathbf{p})= \\
& =-\hat{W}_{-\tau, r_{h} j}\left(R_{h} \mathbf{p}\right) e^{-i \mathbf{p}\left(\boldsymbol{\delta}_{j}-\boldsymbol{\delta}_{1}\right)}=\hat{W}_{\tau, r_{v} j}\left(R_{v} \mathbf{p}\right)=-\hat{W}_{\tau, j}(I \mathbf{p}), \\
\hat{W}_{j, j^{\prime}}(\mathbf{p}) & =\hat{W}_{j+1, j^{\prime}+1}(T \mathbf{p})=\hat{W}_{j, j^{\prime}}^{*}(-\mathbf{p})= \\
& =\hat{W}_{r_{h} j, r_{h} j^{\prime}}\left(R_{h} \mathbf{p}\right) e^{i \mathbf{p}\left(\boldsymbol{\delta}_{j}-\boldsymbol{\delta}_{j^{\prime}}\right)}=\hat{W}_{r_{v} j, r_{v} j^{\prime}}\left(R_{v} \mathbf{p}\right)=\hat{W}_{j, j^{\prime}}(I \mathbf{p}) .
\end{aligned}
$$


Eqs. (CI2)- (C,4) imply that one can define natural covariant matrix elements as:

$$
\begin{aligned}
& \widetilde{W}_{\tau, \tau^{\prime}}(\mathbf{p}):=e^{i \frac{\mathbf{p}}{2} \boldsymbol{\delta}_{1}\left(\tau-\tau^{\prime}\right)} \hat{W}_{\tau, \tau^{\prime}}(\mathbf{p}) \\
& \widetilde{W}_{\tau, j}(\mathbf{p}):=e^{-i \frac{\mathbf{p}}{2}\left(\boldsymbol{\delta}_{j}-\boldsymbol{\delta}_{1}\right)(\tau-1)} \hat{W}_{\tau, j}(\mathbf{p}), \\
& \widetilde{W}_{j, j^{\prime}}(\mathbf{p}):=e^{-i \frac{\mathbf{p}}{2}\left(\boldsymbol{\delta}_{j}-\boldsymbol{\delta}_{j^{\prime}}\right)} \hat{W}_{j, j^{\prime}}(\mathbf{p})
\end{aligned}
$$

which satisfy the following natural transformation rules:

$$
\begin{aligned}
& \left.\widetilde{W}_{\tau, \tau^{\prime}}(\mathbf{p})=\widetilde{W}_{\tau, \tau^{\prime}}(T \mathbf{p})=\widetilde{W}_{\tau, \tau^{\prime}}^{*}(-\mathbf{p})=\widetilde{W}_{-\tau,-\tau^{\prime}}\left(R_{h} \mathbf{p}\right)=\widetilde{W}_{\tau, \tau^{\prime}}\left(R_{v} \mathbf{p}\right)=\widetilde{W}_{\tau, \tau^{\prime}}(I \mathbf{p}), \quad \text { (C) } 8\right) \\
& \widetilde{W}_{\tau, j}(\mathbf{p})=\widetilde{W}_{\tau, j+1}(T \mathbf{p})=-\widetilde{W}_{\tau, j}^{*}(-\mathbf{p})=-\widetilde{W}_{-\tau, r_{h} j}\left(R_{h} \mathbf{p}\right)=\widetilde{W}_{\tau, r_{v} j}\left(R_{v} \mathbf{p}\right)=-\widetilde{W}_{\tau, j}(I \mathbf{p}), \\
& \widetilde{W}_{j, j^{\prime}}(\mathbf{p})=\widetilde{W}_{j+1, j^{\prime}+1}(T \mathbf{p})=\widetilde{W}_{j, j^{\prime}}^{*}(-\mathbf{p})=\widetilde{W}_{r_{h} j, r_{h} j^{\prime}}\left(R_{h} \mathbf{p}\right)=\widetilde{W}_{r_{v} j, r_{v} j^{\prime}}\left(R_{v} \mathbf{p}\right)=\widetilde{W}_{j, j^{\prime}}(I \mathbf{p}) .
\end{aligned}
$$

At first order in $\mathbf{p}$, defining $\widetilde{W}_{\sharp, b}(\mathbf{0})=: a_{\sharp, b}$ and $\partial_{p_{\mu}} \widetilde{W}_{\sharp, b}(\mathbf{0})=: b_{\sharp, b}^{\mu}$, with $\sharp, b \in\{+,-, 1,2,3\}$

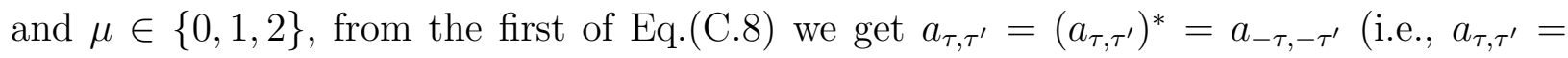
$a+a^{\prime} \tau \tau^{\prime}$, for some $\left.a, a^{\prime} \in \mathbb{R}\right)$ and $b_{\tau, \tau^{\prime}}^{\mu}=0, \forall \mu \in\{0,1,2\}$, that is:

$\widetilde{W}_{\tau, \tau^{\prime}}(\mathbf{p})=a+a^{\prime} \tau \tau^{\prime}+O\left(\mathbf{p}^{2}\right) \quad \Rightarrow \quad \hat{W}_{\tau, \tau^{\prime}}(\mathbf{p})=e^{-i \frac{\vec{p}}{2} \vec{\delta}_{1}\left(\tau-\tau^{\prime}\right)}\left[a+a^{\prime} \tau \tau^{\prime}\right]+O\left(\mathbf{p}^{2}\right), \quad a, a^{\prime} \in \mathbb{R}$,

which proves Eq.(2[27). Similarly, from the second of Eq.(Cl, $)$, we get $a_{\tau, j}=0, b_{\tau, j}^{l}=0$ for $l=1,2$ and $b_{\tau, j}^{0}=b \tau$, for some $b \in \mathbb{R}$, that is

$$
\widetilde{W}_{\tau, j}(\mathbf{p})=b \tau p_{0}+O\left(\mathbf{p}^{2}\right) \quad \Rightarrow \quad \hat{W}_{\tau, j}(\mathbf{p})=b \tau p_{0}+O\left(\mathbf{p}^{2}\right), \quad b \in \mathbb{R},
$$

which proves Eq.(2!28). Finally, from the third of Eq.([C,8), we get that $a_{j, j^{\prime}}=c \delta_{j, j^{\prime}}+c^{\prime}$, for some $c, c^{\prime} \in \mathbb{R}$ and $b_{j, j^{\prime}}^{\mu}=0$, for all $\mu \in\{0,1,2\}$ and $j, j^{\prime} \in\{1,2,3\}$; that is,

$$
\widetilde{W}_{j, j^{\prime}}(\mathbf{p})=c \delta_{j, j^{\prime}}+c^{\prime}+O\left(\mathbf{p}^{2}\right) \quad \Rightarrow \quad \hat{W}_{\tau, j}(\mathbf{p})=e^{i \frac{\vec{p}}{2}\left(\vec{\delta}_{j}-\vec{\delta}_{j^{\prime}}\right)}\left[c \delta_{j, j^{\prime}}+c^{\prime}\right]+O\left(\mathbf{p}^{2}\right), \quad c, c^{\prime} \in \mathbb{R}
$$

which proves Eq.(2,29).

The $A \psi \psi$ kernel. We consider the term quadratic in $\Psi^{(i . r .)}$ and linear in $A_{\sharp}$ in the r.h.s. of Eq.(2.25), which has the form (neglecting the dependence on the label $M$ and defining $\left.\hat{W}_{\sharp}(\mathbf{k}, \mathbf{p}):=\hat{W}_{2,1 ; \sharp}(\mathbf{k}+\mathbf{p}, \mathbf{k}, \mathbf{p})\right):$

$$
\frac{1}{\left(\beta L^{2}\right)^{2}} \sum_{\mathbf{k}, \mathbf{p}, \sigma}\left[\sum_{\tau= \pm} \hat{A}_{\tau, \mathbf{p}} \hat{\Psi}_{\mathbf{k}+\mathbf{p}, \sigma}^{(i . r .)+} \hat{W}_{\tau}(\mathbf{k}, \mathbf{p}) \hat{\Psi}_{\mathbf{k}, \sigma}^{(i . r .)-}+\sum_{j=1,2,3} \hat{A}_{j, \mathbf{p}} \hat{\Psi}_{\mathbf{k}+\mathbf{p}, \sigma}^{(i . r .)+} \hat{W}_{j}(\mathbf{k}, \mathbf{p}) \hat{\Psi}_{\mathbf{k}, \sigma}^{(i . r .)-}\right],
$$


which must be invariant under the symmetry transformations listed in Appendix B. Using symmetries (4)-(8) of Appendix B, we find that:

$$
\begin{aligned}
& \left.\hat{W}_{\tau}(\mathbf{k}, \mathbf{p})=e^{i \tau \frac{\mathbf{p}}{2}\left(\boldsymbol{\delta}_{3}-\boldsymbol{\delta}_{1}\right)} e^{-i(\mathbf{k}+\mathbf{p})\left(\boldsymbol{\delta}_{3}-\boldsymbol{\delta}_{1}\right) \frac{\sigma_{3}}{2}} \hat{W}_{\tau}(T \mathbf{k}, T \mathbf{p}) e^{i \mathbf{k}\left(\boldsymbol{\delta}_{3}-\boldsymbol{\delta}_{1}\right) \frac{\sigma_{3}}{2}}=\hat{W}_{\tau}^{*}(-\mathbf{k},-\mathbf{p})=\text { (C) } 13\right) \\
& \quad=\sigma_{1} \hat{W}_{-\tau}\left(R_{h} \mathbf{k}, R_{h} \mathbf{p}\right) \sigma_{1}=\hat{W}_{\tau}\left(R_{v} \mathbf{k}, R_{v} \mathbf{p}\right)=\hat{W}_{\tau}^{T}(P(\mathbf{k}+\mathbf{p}),-P \mathbf{p})=\sigma_{3} \hat{W}_{\tau}(I \mathbf{k}, I \mathbf{p}) \sigma_{3}, \\
& \hat{W}_{j}(\mathbf{k}, \mathbf{p})=e^{i \frac{\mathbf{p}}{2}\left(\boldsymbol{\delta}_{3}-\boldsymbol{\delta}_{1}\right)} e^{-i(\mathbf{k}+\mathbf{p})\left(\boldsymbol{\delta}_{3}-\boldsymbol{\delta}_{1}\right) \frac{\sigma_{3}}{2}} \hat{W}_{j+1}(T \mathbf{k}, T \mathbf{p}) e^{i \mathbf{k}\left(\boldsymbol{\delta}_{3}-\boldsymbol{\delta}_{1}\right) \frac{\sigma_{3}}{2}}=-\hat{W}_{j}^{*}(-\mathbf{k},-\mathbf{p})= \\
& \quad=-e^{i \mathbf{p}\left(\boldsymbol{\delta}_{j}-\boldsymbol{\delta}_{1}\right)} \sigma_{1} \hat{W}_{r_{h} j}\left(R_{h} \mathbf{k}, R_{h} \mathbf{p}\right) \sigma_{1}=\hat{W}_{r_{v} j}\left(R_{v} \mathbf{k}, R_{v} \mathbf{p}\right)= \\
& \quad=-\hat{W}_{j}^{T}(P(\mathbf{k}+\mathbf{p}),-P \mathbf{p})=-\sigma_{3} \hat{W}_{j}(I \mathbf{k}, I \mathbf{p}) \sigma_{3} .
\end{aligned}
$$

At $\mathbf{k}=\mathbf{p}_{F}^{\omega}$ and $\mathbf{p}=\mathbf{0}$ and defining $\hat{W}_{\tau}\left(\mathbf{p}_{F}^{\omega}, \mathbf{0}\right)=: \sum_{\mu=0}^{3} a_{\omega, \tau}^{\mu} \sigma_{\mu}$, with $\sigma_{0}=1$ and $\sigma_{1}, \sigma_{2}, \sigma_{3}$ the standard Pauli matrices, the last identity in Eq.(C,13) reads:

$$
a_{\omega, \tau}^{0}+a_{\omega, \tau}^{1} \sigma_{1}+a_{\omega, \tau}^{2} \sigma_{2}+a_{\omega, \tau}^{3} \sigma_{3}=a_{\omega, \tau}^{0}-a_{\omega, \tau}^{1} \sigma_{1}-a_{\omega, \tau}^{2} \sigma_{2}+a_{\omega, \tau}^{3} \sigma_{3}
$$

which implies $a_{\omega, \tau}^{1}=a_{\omega, \tau}^{2}=0$; given this fact, the second identity in Eq.(C.13) implies that $a_{\omega, \tau}^{0}=\left(a_{-\omega, \tau}^{0}\right)^{*}$ and $a_{\omega, \tau}^{3}=\left(a_{-\omega, \tau}^{3}\right)^{*}$; the third identity implies that $a_{\omega, \tau}^{0}$ is even in $\tau$, while $a_{\omega, \tau}^{3}$ is odd in $\tau$; the fourth and fifth identity imply that both $a_{\omega, \tau}^{0}$ and $a_{\omega, \tau}^{3}$ are even in $\omega$. In conclusion,

$$
\hat{W}_{\tau}\left(\mathbf{p}_{F}^{\omega}, \mathbf{0}\right)=a^{0}+\tau a^{3} \sigma_{3}
$$

with $a^{0}$ and $a^{3}$ two real constants. This proves Eq.(21:30).

Similarly, defining $\hat{W}_{j}\left(\mathbf{p}_{F}^{\omega}, \mathbf{0}\right)=: \sum_{\mu=0}^{3} a_{\omega, j}^{\mu} \sigma_{\mu}$, the last identity in Eq.(C!14) reads $a_{\omega, j}^{0}+$ $a_{\omega, j}^{1} \sigma_{1}+a_{\omega, j}^{2} \sigma_{2}+a_{\omega, j}^{3} \sigma_{3}=-a_{\omega, j}^{0}+a_{\omega, j}^{1} \sigma_{1}+a_{\omega, j}^{2} \sigma_{2}-a_{\omega, j}^{3} \sigma_{3}$, which implies that $a_{\omega, j}^{0}=a_{\omega, j}^{3}=0 ;$ the first identity in Eq.(C.14) reads:

$$
a_{\omega, j}^{1} \sigma_{1}+a_{\omega, j}^{2} \sigma_{2}=e^{-i \omega \frac{2 \pi}{3} \frac{\sigma_{3}}{2}}\left(a_{\omega, j+1}^{1} \sigma_{1}+a_{\omega, j+1}^{2} \sigma_{2}\right) e^{i \omega \frac{2 \pi}{3} \frac{\sigma_{3}}{2}}
$$

which implies $\left(\begin{array}{c}a_{\omega, j}^{1} \\ a_{\omega, j}^{2}\end{array}\right)=e^{-i \omega \frac{2 \pi}{3} \sigma_{2}}\left(\begin{array}{l}a_{\omega, j+1}^{1} \\ a_{\omega, j+1}^{2}\end{array}\right)$; the second identity in Eq.([C]14) implies that $a_{\omega, j}^{1}=-\left(a_{-\omega, j}^{1}\right)^{*}$ and $a_{\omega, j}^{2}=\left(a_{-\omega, j}^{2}\right)^{*} ;$ the third identity implies that $a_{\omega, j}^{1}=-a_{\omega, r_{h} j}^{1}$ and $a_{\omega, j}^{2}=a_{\omega, r_{h} j}^{2}$; the fourth identity implies that $a_{\omega, j}^{l}=a_{-\omega, r_{v} j}^{l}$, with $l=1,2$; the fifth identity implies that $a_{\omega, j}^{1}=-a_{-\omega, j}^{1}$ and $a_{\omega, j}^{2}=a_{-\omega, j}^{2}$. Using the second, third, fourth and fifth identities for $j=1$ immediately gives $a_{\omega, 1}^{1}=0$ and $a_{\omega, 1}^{2}=a \in \mathbb{R}$. At this point, using the first identity, we get $a_{\omega, 2}^{1}=-a_{\omega, 3}^{1}=\omega a \frac{\sqrt{3}}{2}$ and $a_{\omega, 2}^{2}=a_{\omega, 3}^{2}=-\frac{a}{2}$, which means

$$
\hat{W}_{1}\left(\mathbf{p}_{F}^{\omega}\right)=a \sigma_{2}, \quad \hat{W}_{2}\left(\mathbf{p}_{F}^{\omega}\right)=a\left(\omega \frac{\sqrt{3}}{2} \sigma_{1}-\frac{1}{2} \sigma_{2}\right), \quad \hat{W}_{3}\left(\mathbf{p}_{F}^{\omega}\right)=a\left(-\omega \frac{\sqrt{3}}{2} \sigma_{1}-\frac{1}{2} \sigma_{2}\right)
$$


that is

$$
\hat{W}_{j}\left(\mathbf{p}_{F}^{\omega}, \mathbf{0}\right)=a e^{i \omega \frac{2 \pi}{3}(j-1) \sigma_{3}} \sigma_{2}
$$

which proves Eq.(2131).

Acknowledgements. A.G. and V.M. gratefully acknowledge financial support from the ERC Starting Grant CoMBoS-239694. We thank D. Haldane for valuable discussions on the role of exact lattice Ward Identities.

[1] D. J. Thouless, M. Kohmoto, M. P. Nightingale and M. den Nijs, Phys. Rev. Lett. 49, 405 (1982).

[2] J. E. Avron, R. Seiler and B. Simon, Phys. Rev. Lett. 65, 2185 (1990).

[3] K. Ishikawa and T. Matsuyama, Nucl. Phys. B 280, 523 (1987).

[4] G. W. Semenoff, Phys. Rev. Lett. 53, 2449 (1984).

[5] R. R. Nair et al., Science 320, 1308 (2008); Z. Q. Li et al., Nature Phys. 4, 532 (2008).

[6] T. Ando et al., J. Phys. Soc. Jpn. 71, 1318 (2002); V. P. Gusynin et al., Phys. Rev. Lett. 96, $256802(2006)$.

[7] T. Stauber, N. Peres and A. Geim, Phys. Rev. B 78, 085432 (2008).

[8] I. Herbut, V. Juricic and O. Vafek, Phys. Rev. Lett. 100, 046403 (2008); Phys. Rev. B 82, 235402 (2010); arXiv:0809.0725.

[9] E. G. Mishchenko, Phys. Rev. Lett. 98, 216801 (2007); Europhys. Lett. 83, 17005 (2008).

[10] D. Sheehy and J. Schmalian, Phys. Rev. Lett. 99, 226803 (2007); Phys. Rev. B 80, 193411 (2009).

[11] A. Giuliani and V. Mastropietro, Commun. Math. Phys. 293, 301 (2010).

[12] A. Giuliani and V. Mastropietro, Phys. Rev. B 79, 201403(R) (2009); Phys. Rev. B 82, 199901 (2010).

[13] K. Ziegler, Phys. Rev. B 75, 233407 (2007). 